\title{
A CALIBRATED COMPUTATIONAL FLUID DYNAMICS MODEL FOR SIMULATING THE ROTATING DISK APPARATUS
}

\author{
A Dissertation \\ by \\ ABDELRAHMAN MOSTAFA KOTB \\ Submitted to the Graduate and Professional School of \\ Texas A\&M University \\ in partial fulfillment of the requirements for the degree of \\ DOCTOR OF PHILOSOPHY
}

\begin{abstract}
Chair of Committee, Hadi Nasrabadi
Co-Chair of Committee, Alaa Elwany

Committee Members, Jerome J. Schubert Ibere Nascentes Alves

Head of Department, Jeff Spath
\end{abstract}

August 2021

Major Subject: Petroleum Engineering

Copyright 2021 Abdelrahman Mostafa Kotb 


\begin{abstract}
Reaction kinetics between different rock and acid systems have been studied using the rotating disk apparatus (RDA). However, simplifying assumptions have been made to develop the current equations used to interpret RDA experiments to enable solving them analytically in contrast to using numerical methods. No work has been done to investigate the validity of these assumptions and their impact on the calculation of the reaction kinetics using the RDA.

This work is divided into three main parts: 1) investigating the validity of the assumptions in the equation currently used to interpret RDA results, 2) quantifying the impact of the assumptions on the calculation of the reaction kinetics, and 3) developing a calibrated computational fluid dynamics model to simulate the chemical reaction in the RDA.
\end{abstract}

Chapter II provides insights on some assumptions in the mass transfer of different fluid types in the RDA. Chapter III dives deeper into these assumptions and studies the impact of disk radius on acid turbulence in the reactor. Finally, Chapter IV uses a Gaussian based proxy model to develop the first calibrated computational fluid dynamics model to calculate the diffusion coefficient and reaction rate constant between hydrochloric acid and calcite rock.

The main findings are: 1) Current RDA reactor dimensions are not large enough to prevent the impact of the boundaries on the mass transfer of $\mathrm{H}^{+}$to the disk, which can increase $\mathrm{H}^{+}$mass transfer to the disk of up to $28 \%, 2$ ) the critical Reynolds number for the flow at the surface of the disk is in the range $1-2 \times 10^{4}$ and not in the $10^{5}$ as previously 
reported in the literature, and 3) the developed calibrated surrogate model can predict the diffusion coefficient with an improvement in prediction accuracy obtained through experimental validation of $63 \%$ over Newman's conventional method. 


\section{DEDICATION}

To my parents, Dr. Hala and Dr. Mostafa, my wife Lina, my children Ali and Judy, and the late Dr. Hisham A. Nasr-El-Din, may his soul rest in peace. 


\section{ACKNOWLEDGEMENTS}

I would like to thank God for his guidance through this journey, as without him, none of this would have been possible. I would like to thank my committee chair, Dr. Hadi Nasrabadi, and my committee members, Dr. Jerome Schubert, Dr. Alaa Elwany, and Dr. Ibere Alves, for their valuable guidance and support throughout this research. I would also like to recognize Ms. Gia Alexander for proofreading and editing all written work pertaining to my research.

To Dr. Hisham Nasr-El-Din, my original committee chair, may he rest in peace: Thank you for your treasured advice, involvement, and dedication to this work and to my success throughout the past few years.

To my colleagues: I truly value the advice and knowledge sharing that transpired over the years. I want to thank Dr. Mahmoud Ali, Dr. Igor Ivanishin, and Dr. Khatere Sokhanvarian for being great colleagues who supported this work and kept pushing me to be better. I would like to thank John Maldonado for his hard work maintaining and fixing lab equipment through the years. He has genuinely saved this work countless times. I would also like to thank the high-performance computing facility at Texas A\&M for their continuous support throughout my work.

Finally, to my parents, wife, and siblings who kept believing in me even when I kept doubting myself. I want to thank them. 


\section{CONTRIBUTORS AND FUNDING SOURCES}

\section{Contributors}

This work was supervised by a dissertation committee consisting of Professors Hisham A. Nasr-El-Din and Hadi Nasrabadi, committee co-advisors, Professors Jerome J. Schubert and Ibere Nascentes Alves of the Harold Vance Department of Petroleum Engineering, and Professor Alaa Elwany of the Industrial Engineering at Texas A\&M University.

Experimentation in Chapters II and III were performed in collaboration with Dr. Igor Ivanishin. Modeling was initiated in collaboration with Dr. Mahmoud Ali. All other work conducted for this dissertation was completed by the student independently.

\section{Funding Sources}

Graduate study was supported in part by various teaching and research assistantships from the Texas A\&M University College of Engineering and the Harold Vance Department of Petroleum Engineering. 


\section{ACRONYMS}

$\begin{array}{ll}\text { CFD } & \text { Computational Fluid Dynamics } \\ \text { GP } & \text { Gaussian process } \\ \mathrm{HCl} & \text { Hydrochloric Acid } \\ \text { ICP } & \text { Inductively Coupled Plasma } \\ \text { LHD } & \text { Latin Hypercube Design } \\ \text { MLE } & \text { Maximum Likelihood Estimation } \\ \text { MSE } & \text { Mean Square Error } \\ \text { RDA } & \text { Rotating Disk Apparatus } \\ \text { RPM } & \text { Hydrochloric Acid } \\ \text { PAP } & \text { Percentage of Accuracy-Precision }\end{array}$

vii 


\section{NOMENLCLATURE}

$$
\begin{aligned}
& A_{d} \quad=\text { initial area of the disk exposed to acid, } \mathrm{cm}^{2} \\
& a^{\prime} \quad=\text { constant, - } \\
& A=\text { dimensionless radial velocity gradient at the disk surface } \\
& B \quad=\text { constant } \\
& C_{b} \quad=\text { bulk reactant concentration, fraction } \\
& C_{r}=\text { total calcium ion concentration in the disk, gmol } \\
& C_{s} \quad=\text { reactant concentration on the disk, fraction } \\
& D_{e} \quad=\text { diffusion coefficient, } \mathrm{m}^{2} / \mathrm{s} \\
& e_{i} \quad=\text { independent and identically zero-mean normally distributed random variable } \\
& F \quad=\text { Faraday's constant, s.A } / \mathrm{mol} \\
& f \quad=\quad \text { sum of external body forces, } \mathrm{N} \\
& I=\text { current density, } \mathrm{A} / \mathrm{m}^{2} \\
& J_{m t}=\text { rate of mass transfer, gmol } / \mathrm{cm}^{2} / \mathrm{s} \\
& K=\text { reaction rate coefficient, } \mathrm{m} / \mathrm{s} \\
& K=\text { positive-definite parametric covariance function } \\
& K^{\prime}=\text { power-law consistency index, } \mathrm{g} \cdot \mathrm{s}^{(\mathrm{n}-2)} / \mathrm{cm} \\
& L \quad=\text { dimension } \\
& M \quad=\text { molarity } \\
& N=\text { number of electrons produced } \\
& n=\text { reaction order } \\
& n^{c} \quad=\text { number of simulations performed }
\end{aligned}
$$




$$
\begin{aligned}
& n^{p} \quad=\text { number of experiments } \\
& n^{t} \quad=\text { number of points reserved for testing } \\
& n^{\prime} \quad=\text { power-law behavior index } \\
& p \quad=\text { pressure, } \mathrm{psi} \\
& R_{e} \quad=\text { Reynolds number } \\
& R e_{c r}=\text { critical Reynolds number } \\
& R_{e h}=\frac{\omega y^{2}}{v} \\
& R_{e h r}=\frac{\omega y r_{r}}{v} \\
& r \quad=\text { radial distance from the center of rotation, } \mathrm{m} \\
& r^{\prime} \quad=\text { radius of the disk, } \mathrm{cm} \\
& \mathrm{r}_{r}=\text { radius of the reactor, } \mathrm{cm} \\
& S_{c} \quad=\text { Schmidt number } \\
& S_{m} \quad=\quad \text { source mass added to the continuity equation } \\
& t \quad=\text { time, } \mathrm{s} \\
& t_{r} \quad=\quad \text { Ion transport number } \\
& u=\text { superficial velocity vector, } \mathrm{m} / \mathrm{s} \\
& v \quad=\text { kinematic viscosity, } \mathrm{m}^{2} / \mathrm{s} \\
& V_{y} \quad=\text { acid velocity in the } \mathrm{y} \text { direction, } \mathrm{m} / \mathrm{s} \\
& x=\text { vector of input variables } \\
& y=\text { axial distance from the disk to the bottom of the reactor, } \mathrm{m} \\
& y^{p} \quad=\text { physical response, fraction }
\end{aligned}
$$




$$
\begin{array}{ll}
\hat{y}^{c} & =\text { surrogate model response, fraction } \\
Y^{c} & =\text { CFD response vector } \\
\alpha & =\text { sill parameter } \\
\alpha^{\prime} & =\text { amount of rock dissolved, fraction } \\
\beta_{0} & =\text { error term } \\
\delta & =\text { bias correction term } \\
\delta^{\prime} & =\text { thickness of diffusion boundary layer, } \mu \mathrm{m} \\
\delta_{0} & =\text { thickness of hydrodynamic boundary layer, } \mu \mathrm{m} \\
\eta & =\text { zero mean Gaussian process } \\
\vartheta & =\text { kernel function } \\
\lambda & =\text { smoothing parameters } \\
\aleph_{\vartheta} & =\text { native space } \\
\rho g & =\text { gravitational body force, } \mathrm{N} \\
\Sigma & =\text { covariance matrix } \\
\sigma & =n^{c} \times 1 \text { vector of covariances } \\
\tau & =\text { stress, psi } \\
& =\text { porosity, fraction } \\
\sigma &
\end{array}
$$




\section{TABLE OF CONTENTS}

Page

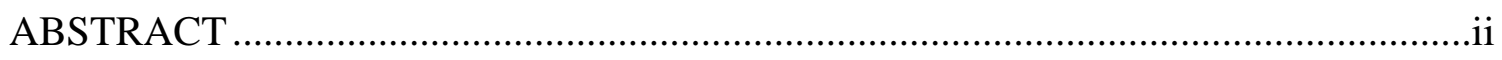

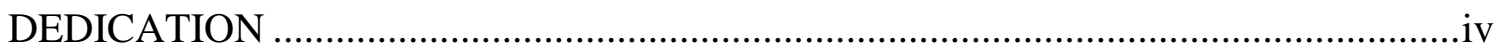

ACKNOWLEDGEMENTS ...............................................................................

CONTRIBUTORS AND FUNDING SOURCES .....................................................vi

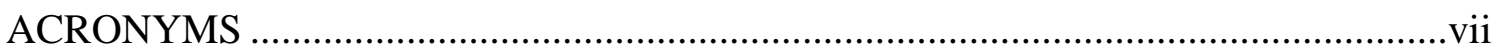

NOMENLCLATURE ..................................................................................... viii

TABLE OF CONTENTS ...................................................................................

LIST OF FIGURES ....................................................................................... xiii

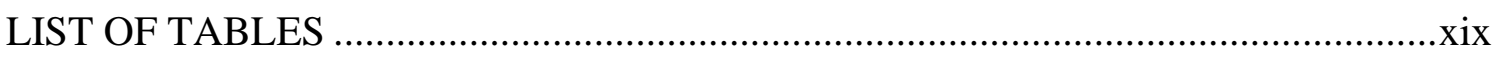

CHAPTER I INTRODUCTION .................................................................. 1

Mass Transfer When Using the Rotating Disk Apparatus for Newtonian and Non-

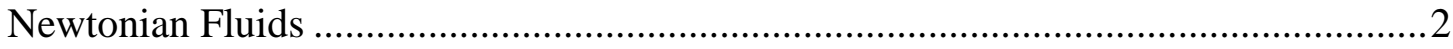

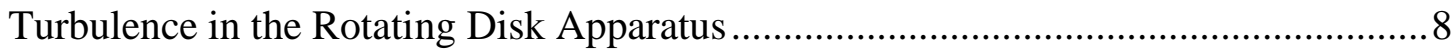

Computational Fluid Dynamics Model for Simulating the Rotating Disk Apparatus . 12

CHAPTER II NEW INSIGHTS INTO MASS TRANSFER WHEN USING THE ROTATING DISK APPARATUS FOR NEWTONIAN AND NON-NEWTONIAN

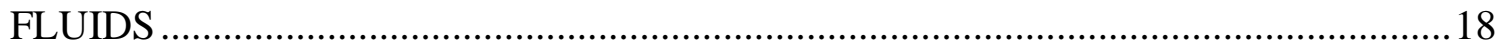

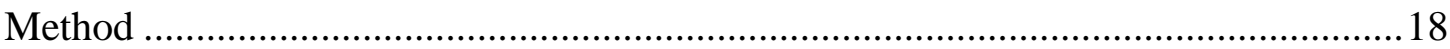

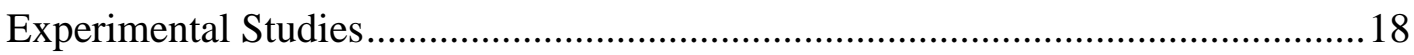

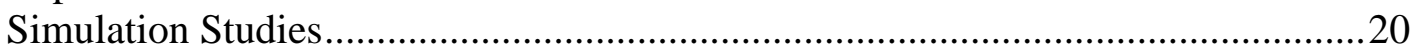

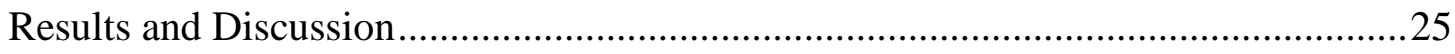

Non-Newtonian Fluid Properties and Velocity Profile .........................................25

Impact of Reactor Dimensions on the Fluid Flow in Newtonian Fluids ..................26

Impact of Reactor Dimensions on the Fluid Flow in Non-Newtonian Fluids...........34

Impact of Reactor Dimensions on the Mass Transfer of $\mathrm{H}^{+}$to the Disk in

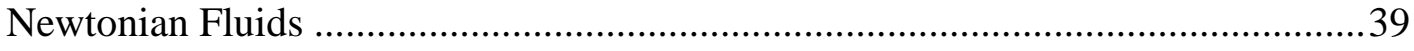

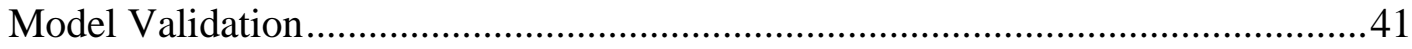




\section{CHAPTER III TURBULENCE LEADS TO OVERESTIMATION OF THE ACID- DIFFUSION COEFFICIENT AT TYPICAL EXPERIMENTAL CONDITIONS

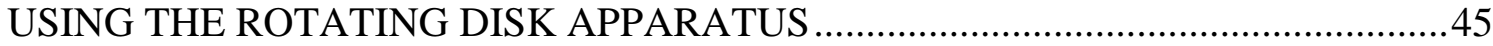

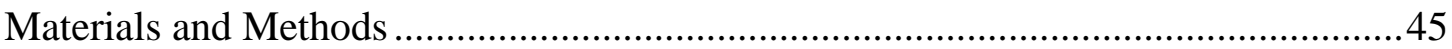

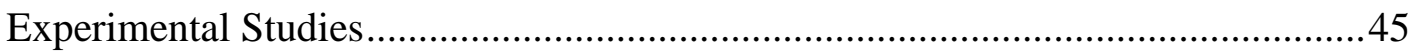

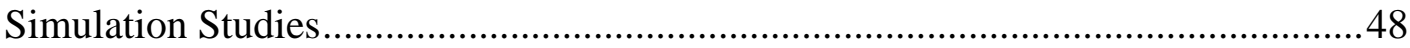

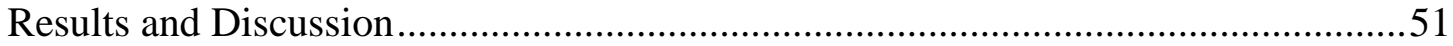

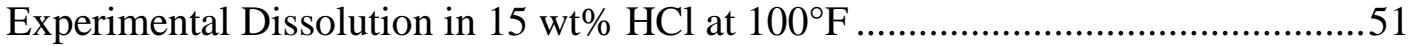

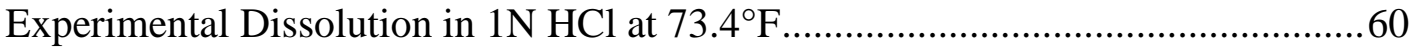

Simulation: Impact of Disk size on the Disk Dissolution Rate and Velocity

Profile .66

CHAPTER IV A CALIBRATED COMPUTATIONAL FLUID DYNAMICS MODEL FOR SIMULATING THE ROTATING DISK APPARATUS ...................... 70

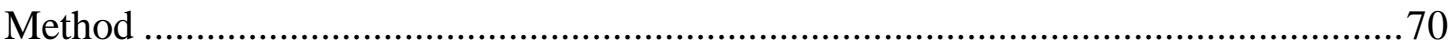

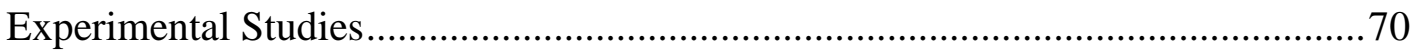

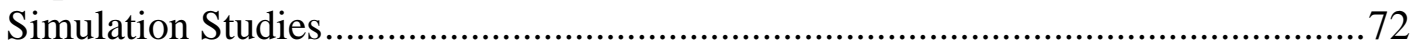

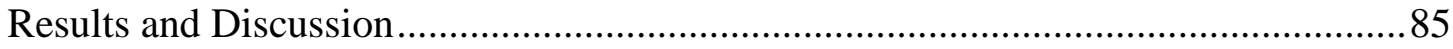

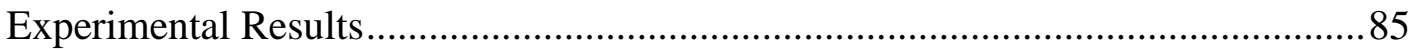

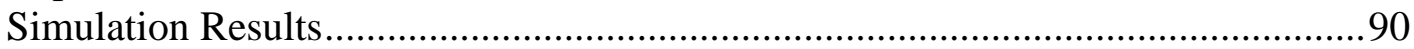

CHAPTER V CONCLUSIONS, RECOMMENDATIONS AND FUTURE WORK...101

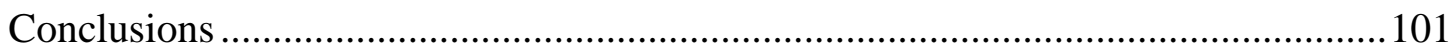

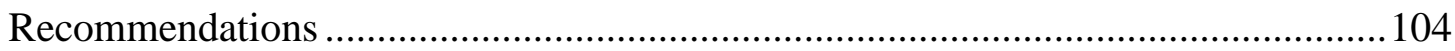

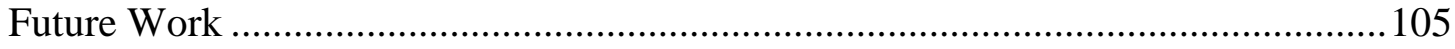

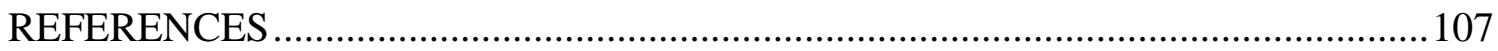




\section{LIST OF FIGURES}

Fig. II-1 A schematic diagram for the rotating disk. Reprinted with permission from (Kotb and Nasr-El-Din 2020).

Fig. II-2 Effect of the number of cells in the five reactors simulated on the rock dissolved for five minutes. Reprinted with permission from (Kotb and NasrEl-Din 2020).

Fig. II-3 Effect of polymer concentration on the viscosity of polymer solutions. Reprinted with permission from (Kotb and Nasr-El-Din 2020).

Fig. II-4 Petroleum-based dye (red) showing reverse flow under the disk for $0.1 \mathrm{wt} \%$ polymer solution at $1,800 \mathrm{rpm}$. Reprinted with permission from (Kotb and Nasr-El-Din 2020).

Fig. II-5 Velocity profile for reactor A with $2 \mathrm{cp} \mathrm{HCl}\left(36 \mathrm{wt} \%\right.$ at $70^{\circ} \mathrm{F}$ Nishikata et al. 1981) at $300 \mathrm{~s}$ with $100 \mathrm{rpm}$. Reactor height is $9.3 \mathrm{~cm}$ and reactor diameter is $8 \mathrm{~cm}$. Axial distance between the base of the disk and the bottom of the reactor is $3.2 \mathrm{~cm}$. Axisymmetric flow is observed. Reprinted with permission from (Kotb and Nasr-El-Din 2020).

Fig. II-6 Velocity profile for reactor A with $1 \mathrm{cp} \mathrm{HCl}\left(15 \mathrm{wt} \%\right.$ at $86^{\circ} \mathrm{F}$ Nishikata et al. 1981) at $300 \mathrm{~s}$ with $100 \mathrm{rpm}$. Reactor height is $9.3 \mathrm{~cm}$ and reactor diameter is $8 \mathrm{~cm}$. Axial distance between the base of the disk and the bottom of the reactor is $3.2 \mathrm{~cm}$. Transition from axisymmetric flow is observed. Reprinted with permission from (Kotb and Nasr-El-Din 2020).

Fig. II-7 Velocity profile for reactor A with $0.5 \mathrm{cp} \mathrm{HCl}\left(15 \mathrm{wt} \%\right.$ at $150^{\circ} \mathrm{F}$ Nishikata et al. 1981) at $300 \mathrm{~s}$ with $1,000 \mathrm{rpm}$. Reactor height is $9.3 \mathrm{~cm}$ and reactor diameter is $8 \mathrm{~cm}$. Axial distance between the base of the disk and the bottom of the reactor is $3.2 \mathrm{~cm}$. Asymmetric flow is observed. Reprinted with permission from (Kotb and Nasr-El-Din 2020).

Fig. II-8 Velocity profile for reactor B with $1 \mathrm{cp} \mathrm{HCl}\left(15 \mathrm{wt} \%\right.$ at $86^{\circ} \mathrm{F}$ Nishikata et al. 1981) at $300 \mathrm{~s}$ with $100 \mathrm{rpm}$. Reactor height is $9.3 \mathrm{~cm}$ and reactor diameter is $11.4 \mathrm{~cm}$. Axial distance between the base of the disk and the bottom of the reactor is $3.2 \mathrm{~cm}$. Asymmetric flow is observed. Reprinted with permission from (Kotb and Nasr-El-Din 2020).

Fig. II-9 Velocity profile for reactor $\mathrm{C}$ with $1 \mathrm{cp} \mathrm{HCl}\left(15 \mathrm{wt} \%\right.$ at $86^{\circ} \mathrm{F}$ Nishikata et al. $1981)$ at $300 \mathrm{~s}$ with $100 \mathrm{rpm}$. Reactor height is $13.4 \mathrm{~cm}$ and reactor diameter is $8 \mathrm{~cm}$. Axial distance between the base of the disk and the bottom of the 
reactor is $7.3 \mathrm{~cm}$. Asymmetric flow is observed. Reprinted with permission from (Kotb and Nasr-El-Din 2020).

Fig. II-10 Effect of shear rate on the apparent viscosity of gelled acid (1.5 wt $\%$ polymer concentration and $5 \mathrm{wt} \% \mathrm{HCl}$ ) at $122^{\circ} \mathrm{F}$ (Nasr-El-Din et al. 2008). Reprinted with permission from (Kotb and Nasr-El-Din 2020).

Fig. II-11 Velocity profile for reactor A with gelled acid (1.5 wt\% polymer concentration and $5 \mathrm{wt} \% \mathrm{HCl}$ at $122^{\circ} \mathrm{F}$ Nasr-El-Din et al. 2008) at $300 \mathrm{~s}$ with $100 \mathrm{rpm}$. Reactor height is $9.3 \mathrm{~cm}$ and reactor diameter is $8 \mathrm{~cm}$. Axial distance between the base of the disk and the bottom of the reactor is $3.2 \mathrm{~cm}$. Axisymmetric flow is observed. Reprinted with permission from (Kotb and Nasr-El-Din 2020).

Fig. II-12 Strain profile for gelled acid (1.5 wt $\%$ polymer concentration and $5 \mathrm{wt} \% \mathrm{HCl}$ at $122^{\circ} \mathrm{F}$ Nasr-El-Din et al. 2008) in reactor A at $300 \mathrm{~s}$ with $100 \mathrm{rpm}$. Reactor height is $9.3 \mathrm{~cm}$ and reactor diameter is $8 \mathrm{~cm}$. Axial distance between the base of the disk and the bottom of the reactor is $3.2 \mathrm{~cm}$. Reprinted with permission from (Kotb and Nasr-El-Din 2020).

Fig. II-13 Velocity profile for reactor A with gelled acid (1.5 wt\% polymer concentration and $5 \mathrm{wt} \% \mathrm{HCl}$ at $122^{\circ} \mathrm{F}$ Nasr-El-Din et al. 2008) at $300 \mathrm{~s}$ with $2,100 \mathrm{rpm}$. Reactor height is $9.3 \mathrm{~cm}$ and reactor diameter is $8 \mathrm{~cm}$. Axial distance between the base of the disk and the bottom of the reactor is $3.2 \mathrm{~cm}$. Transition from axisymmetric flow is observed. Reprinted with permission from (Kotb and Nasr-El-Din 2020).

Fig. II-14 Velocity profile for case B with gelled acid (1.5 wt $\%$ polymer concentration and $5 \mathrm{wt} \% \mathrm{HCl}$ at $122^{\circ} \mathrm{F}$ Nasr-El-Din et al. 2008) at $300 \mathrm{~s}$ with 1,500 rpm. Reactor height is $9.3 \mathrm{~cm}$ and reactor diameter is $11.4 \mathrm{~cm}$. Axial distance between the base of the disk and the bottom of the reactor is $3.2 \mathrm{~cm}$. Transition from axisymmetric flow is observed. Reprinted with permission from (Kotb and Nasr-El-Din 2020).

Fig. II-15 Rate of dissolution of the disk inside $15 \mathrm{wt} \% \mathrm{HCl}$ in the five reactors. Parameters discussed in Table II-2. Reprinted with permission from (Kotb and Nasr-El-Din 2020).

Fig. II-16 Marble disks from RDA experiments performed at $150^{\circ} \mathrm{F}$ using $15 \mathrm{wt} \% \mathrm{HCl}$ and $1 \mathrm{wt} \%$ corrosion inhibitor. The disks rotational speeds from left to right were 132, 584, and 1,172 rpm. Reprinted with permission from (Kotb and Nasr-El-Din 2020).

Fig. II-17 $\mathrm{Ca}^{+2}$ dissolved for the RDA experiment performed at $150^{\circ} \mathrm{F}$ using $15 \mathrm{wt} \%$ $\mathrm{HCl}$ and $1 \mathrm{wt} \%$ corrosion inhibitor. Results obtained from ICP xiv 
measurements. Reprinted with permission from (Kotb and Nasr-El-Din 2020).

Fig. II-18 Comparison between the rate of dissolution of the disk inside $15 \mathrm{wt} \% \mathrm{HCl}$ in the five reactors (parameters discussed in Table II-2) and the experimental results performed at the same conditions with reactor A dimensions. Reprinted with permission from (Kotb and Nasr-El-Din 2020).

Fig. II-19 Comparison between the rate of dissolution of the disk inside $18.76 \mathrm{wt} \% \mathrm{HCl}$ in the five reactors (parameters discussed in Table II-2) and the experimental results obtained by Arslan et al. (2017) with reactor A dimensions. Reprinted with permission from (Kotb and Nasr-El-Din 2020).

Fig. III-1 A schematic diagram for the rotating disk. Reprinted with permission from (Ivanshin et al. 2021).

Fig. III-2 Mass-transfer rate to the disks of calcite marble as a function of the disk rotational speed in $15 \mathrm{wt} \% \mathrm{HCl}$ at $100^{\circ} \mathrm{F}$. Reprinted with permission from (Ivanshin et al. 2021).

Fig. III-3 Disks of calcite marble after the dissolution experiments. Moving forward from back - disks of 1.46, 1.11, and 0.72 in. diameter. Moving from left to right - disk rotational speed of 207, 380, 587, 829, 1,175, and 1,555 rpm. Reprinted with permission from (Ivanshin et al. 2021).

Fig. III-4 Distribution of streamlines at the surface of the RD: (a) schematic; (b) etched lines on the RD (after Levich 1962); (c) 1.46 in. disk after RDA test at 380 rpm (present study). Reprinted with permission from (Ivanshin et al. 2021)...54

Fig. III-5 Progressive loss of uniform accessibility of the surface of 1.46 in. disk during RDA tests at $587 \mathrm{rpm}$ (a) and 1,555 rpm (b). Note several comparably large cavities at the outer region of the disk in (a), and smaller size cavities covering the most of the disk surface with smooth hump at the center of the disk in (b). Reprinted with permission from (Ivanshin et al. 2021).

Fig. III-6 1.46 in. disks after duplicate RDA tests at $587 \mathrm{rpm}$. Reprinted with permission from (Ivanshin et al. 2021).

Fig. III-7 Transition from laminar to turbulent regime in boundary layer flow: (a) schematic structure of the transition regime with spiral and ring-like vortices, (b) the photograph showing three regions on the surface of the disk - laminar in the center, transition with many spiral vortices, and turbulent with irregular flow pattern (after Kohama 1984). Reprinted with permission from (Ivanshin et al. 2021). 
Fig. III-8 1.46 in. disk after the dissolution test showing the regions of laminar, transition, and turbulent flow regimes in the boundary layer. Note the similarity with Fig. 6(b). Reprinted with permission from (Ivanshin et al. 2021).

Fig. III-9 Concentration of calcium ion in the fluid samples withdrawn during the RDA test at $1,555 \mathrm{rpm}$ using the disk of 1.46 in. diameter. The first four data points were used to calculate the mass-transfer rate to the disk surface. Reprinted with permission from (Ivanshin et al. 2021)

Fig. III-10 Mass-transfer rate to the disks of calcite marble as a function of the disk rotational speed in $15 \mathrm{wt} \% \mathrm{HCl}$ at $100^{\circ} \mathrm{F}$. Trendlines including only the filled markers, i.e. the data points in laminar flow regime. Reprinted with permission from (Ivanshin et al. 2021)

Fig. III-11 1.46 in. disks composed of grains of maximum size of $1000 \mu \mathrm{m}$ after dissolution in $1 \mathrm{~N} \mathrm{HCl}$ at $73.4^{\circ} \mathrm{F}$, and disk rotational speeds of 518 (left) and 1,175 (right) rpm. Reprinted with permission from (Ivanshin et al. 2021).....64

Fig. III-12 1.46 in. disks composed of grains of maximum size of $150 \mu \mathrm{m}$ after dissolution in $1 \mathrm{~N} \mathrm{HCl}$ at $73.4^{\circ} \mathrm{F}$, and disk rotational speeds of 518 (left) and 1,175 (right) rpm. Reprinted with permission from (Ivanshin et al. 2021).....64

Fig. III-13 Mass-transfer rate to the disks of calcite marble as a function of the disk rotation speed in $1 \mathrm{~N} \mathrm{HCl}$ at $73.4^{\circ} \mathrm{F}$. Reprinted with permission from (Ivanshin et al. 2021).

Fig. III-14 Side view of the disks comparing the velocity profiles at different disk size and rotational speed at five minutes. Dissolution in $15 \mathrm{wt} \% \mathrm{HCl}$ at $100^{\circ} \mathrm{F}$ was simulated. Reprinted with permission from (Ivanshin et al. 2021).

Fig. IV-1 Schematic for the rotating disk apparatus. Reprinted with permission from (Kotb et al. 2021).

Fig. IV-2 Density data for different molarity $\mathrm{HCl}$. All Acids contained $1 \mathrm{wt} \%$ corrosion inhibitor. Reprinted with permission from (Kotb et al. 2021).

Fig. IV-3 Kinematic viscosity data for different molarity $\mathrm{HCl}$. All Acids contained 1 wt \% corrosion inhibitor. Reprinted with permission from (Kotb et al. 2021). 71

Fig. IV-4 $\mathrm{HCl}$ concentration used for the LHD ranged from 0.27 to $4.38 \mathrm{M}$. Reprinted with permission from (Kotb et al. 2021).

Fig. IV-5 Disk rotational speed used for the LHD ranged from 110 to $993 \mathrm{rpm}$. Reprinted with permission from (Kotb et al. 2021). 
Fig. IV-6 Diffusion Coefficient used for the LHD ranged from 1.66E-9 to $1.09 \mathrm{E}-7 \mathrm{~m} / \mathrm{s}$. Reprinted with permission from (Kotb et al. 2021)....................................78

Fig. IV-7 Reaction rate coefficient used for the LHD ranged from 5.7E-3 to $1.4 \mathrm{~m} / \mathrm{s}$. Reprinted with permission from (Kotb et al. 2021) ........................................78

Fig. IV-8 Acid kinematic viscosity used for the LHD ranged from 0.25 to 1.1 cst........79

Fig. IV-9 Initial porosity of disk used for the LHD ranged from 0 to $14 \%$. Reprinted with permission from (Kotb et al. 2021). .......................................................79

Fig. IV-10 Marble disks after the RDA experiments. Reprinted with permission from

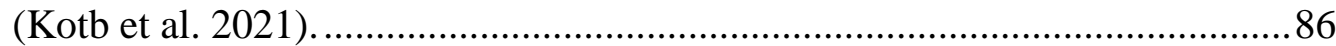

Fig. IV-11 Indiana limestone disks after the RDA experiments. Reprinted with permission from (Kotb et al. 2021) .............................................................. 87

Fig. IV-12 Rate of $\mathrm{H}^{+}$mass transfer to the disk obtained from the ICP data showing the impact of temperature at $4.83 \mathrm{M} \mathrm{HCl}$. Reprinted with permission from (Kotb et al. 2021).

Fig. IV-13 Rate of $\mathrm{H}^{+}$mass transfer to the disk obtained from the ICP data showing the impact of $\mathrm{HCl}$ concentration at $250^{\circ} \mathrm{F}$. Reprinted with permission from (Kotb et al. 2021).

Fig. IV-14 Bias between the RDA experiments and CFD model runs. Reprinted with permission from (Kotb et al. 2021).

Fig. IV-15 Outputs from the RDA experiments versus CFD model runs as a function of concentration. Reprinted with permission from (Kotb et al. 2021).............92

Fig. IV-16 Outputs from the RDA experiments versus CFD model runs as a function of acid viscosity. Reprinted with permission from (Kotb et al. 2021).

Fig. IV-17 The predictions from the proxy model at the 80 design points as a function of concentration. Reprinted with permission from (Kotb et al. 2021)..............95

Fig. IV-18 The predictions from the proxy model at the 80 design points as a function of acid viscosity. Reprinted with permission from (Kotb et al. 2021). ............95

Fig. IV-19 Boxplots of the MSE from 100 samples using the developed model (on the left) versus Newman's calculation (1966) (on the right). Reprinted with permission from (Kotb et al. 2021). 
Fig. IV-20 Boxplots comparison of the PAP of dissolved rock from 100 samples using the developed model (on the left) versus Newman's calculation (1966) (on the right). Reprinted with permission from (Kotb et al. 2021)........................100 


\section{LIST OF TABLES}

Table I-1 Acid volumes used in the RDA reported in the literature. Reprinted with permission from (Kotb and Nasr-El-Din 2020).

Table II-1 Dimensions of the simulated reactors and the number of cells. Experiments were performed in Reactor A. Reprinted with permission from (Kotb and Nasr-El-Din 2020).

Table II-2 Parameters for the simulation run for the different tests performed in this work. For the mass transfer rate and maximum radial velocity, the acid density was measured using a density meter. The acid viscosity was obtained from Nishikata et al. (1981), the diffusion coefficient was obtained from Qiu et al. (2015), and the reaction rate coefficient was obtained from Peng et al. (2015). For flow asymmetry, fluid density and viscosity were obtained from Nishikata et al. (1981). For Matching Arslan et al. (2017), the temperature, acid concentration, acid density, acid viscosity, and diffusion coefficient were reported by Arslan et al. (2017). The reaction rate coefficient was calculated based on the activation energy reported by Arslan et al. (2017) and the reaction rate constant reported by Peng et al. (2015). Reprinted with permission from (Kotb and Nasr-El-Din 2020).

Table II-3 Different flow regimes observed with their respective $R_{\text {ehr }}$ value. The $R_{\text {ehr }}$ value range calculated in the case of gelled acid represents the values if the zero and infinite shear viscosity were used in the calculation of $R_{\text {ehr, }}$, respectively. Maximum radial velocity and Dissolved disk in the different reactors. The simulations were performed under the conditions discussed in Table II-2. Reprinted with permission from (Kotb and Nasr-El-Din 2020).....33

Table III-1 Number of cells used for the different disk sizes. Reprinted with permission from (Ivanshin et al. 2021).

Table III-2 Thicknesses of the hydrodynamic and diffusion boundary layers. Reprinted with permission from (Ivanshin et al. 2021)....

Table III-3 Slope of the plot of rate of mass transfer of $\mathrm{H}^{+}$as a function of square root of disk rotational speed at three disk diameters showing a direct relationship between the mass transfer of $\mathrm{H}^{+}$and disk diameter. Reprinted with permission from (Ivanshin et al. 2021).

Table IV-1 The different tests performed in the RDA and the results obtained.

Reprinted with permission from (Kotb et al. 2021). 
Table IV-2 Calculated diffusion coefficient using Newman's (1966) equation. The estimated diffusion coefficient and rection rate coefficient estimated from the model. Reprinted with permission from (Kotb et al. 2021)...........................89

Table IV-3 Comparison between the dissolved rock obtained using Newman's (1966) equation, the proposed model and the actual experimental value. Reprinted with permission from (Kotb et al. 2021). 


\section{CHAPTER I \\ INTRODUCTION ${ }^{* \dagger \dagger}$}

To improve oil and gas production, petroleum engineers perform well stimulation using well fracturing and well acidizing. This dissertation focuses on acid stimulation. Specifically, calculating the reaction kinetics using the rotating disk apparatus (RDA). Reaction kinetics provides insights on acid volume and injection rate to be used in the field.

The RDA was introduced in the oil and gas industry in the middle of the $20^{\text {th }}$ century, and the equation used to interpret the RDA has not been changed since Newman introduced it in 1966. This equation has inherent assumptions. The validity and the impacts of these assumptions have not been discussed in the literature. As a result, this dissertation is divided into three main chapters discussing the RDA. Chapter II provides insights on some assumptions in the mass transfer of different RDA fluid types. Chapter III dives deeper into these assumptions and studies the impact of disk radius on acid turbulence in the reactor. Finally, Chapter IV uses a Gaussian-based proxy model to develop the first

\footnotetext{
* Part of this chapter is reprinted with permission from "New Insights into Mass Transfer When Using the Rotating Disk Apparatus for Newtonian and Non-Newtonian Fluids" by Kotb, A. and Nasr-El-Din, H. A., 2020, SPE J, Copyright 2020 by Society of Petroleum Engineers.

${ }^{\dagger}$ Part of this chapter is reprinted with permission from "Turbulence Leads to Overestimation of the AcidDiffusion Coefficient at Typical Experimental Conditions using the Rotating Disk Apparatus" by Ivanshin, I., Kotb, A., and Nasr-El-Din 2021, Journal of Petroleum Science and Engineering 205. Copyright 2021 by Elsevier.

* Part of this chapter is reprinted with permission from "A Calibrated Computational Fluid Dynamics Model for Simulating the Rotating Disk Apparatus" by Kotb, A. et al. 2021, SPE J, Copyright 2021 by Society of Petroleum Engineers.
} 
calibrated computational fluid dynamics model to calculate the diffusion coefficient and reaction rate constant between hydrochloric acid and calcite rock. Literature for each chapter is presented below.

\section{Mass Transfer When Using the Rotating Disk Apparatus for Newtonian and Non- Newtonian Fluids}

Formation damage occurs in drilling, completion, and workover operations. This damage creates a zone near the wellbore with low permeability that negatively affects production. A common solution to this problem is matrix acidizing, in which acid is injected below the fracture pressure. In the case of carbonate reservoirs, the acid creates high permeability channels known as wormholes. These wormholes remediate permeability and restore production rates.

Understanding reaction kinetics between the rock and acid is necessary to optimize the acidizing process. The rotating disk apparatus (RDA) has been used to study reaction kinetics between rocks and Newtonian and non-Newtonian fluids (Hansford and Litt 1968; Boomer et al. 1972; Lund et al. 1973; Roberts and Guin 1975; Prakongpan et al. 1976; Anderson 1991; Fredd and Fogler 1998; Alkattan et al. 1998; Conway et al. 1999; Taylor and Nasr-El-Din 2009; Rabie et al. 2014; Li et al. 2016; Liao et al. 2017; Abdelgawad et al. 2018; Kotb et al. 2018; Sayed et al. 2020). However, no standard exists in the literature for the acid volume used in RDA experiments. The present discussion seeks to establish that standard beginning with values that have been reported previously. Table I-1 lists the acid volumes used in the RDA reported in the literature. 
Acid Volume Used in the

Reactor $\left(\mathrm{cm}^{3}\right)$

450

500

700

975

1,000

1,800
Reference

Kotb et al. 2018

Abdelgawad et al. 2018

Sayed et al. 2020

Boomer et al. 1972

Rashed et al. 2016

Alkattan et al. 1998

Table I-1 Acid volumes used in the RDA reported in the literature. Reprinted with permission from (Kotb and Nasr-El-Din 2020).

Newman (1966) developed Eq. 1, which is currently used to calculate the diffusion coefficient from the RDA for Newtonian fluids:

$J_{m t}=\frac{0.62048 S_{c}^{-\frac{2}{3}}(v \omega)^{0.5}}{1+0.298 S_{c}^{-\frac{1}{3}}+0.1451 S_{c}^{-\frac{2}{3}}}\left(C_{b}-C_{S}\right)$

where $J_{m t}$ is the rate of mass transfer of $\mathrm{H}^{+}$to the rotating disk, $S_{c}$ is Schmidt number, which is defined as the ratio between the kinematic viscosity and the diffusion coefficient of a fluid, $v$ is the kinematic viscosity of the fluid, $\omega$ is the rotational disk speed, $C_{b}$ is the bulk reactant concentration, and $C_{s}$ is the reactant concentration on the disk surface.

Eq. 1 is based on the following assumptions:

- The surface area of the disk and fluid properties (acid concentration, density, and viscosity) remain the same throughout the experiment.

- The flow is single-phase and laminar. The laminar flow is defined by a Reynolds number lower than $10^{5}$ (Levich 1962). 
- The reactor boundaries are assumed infinite so as not to impact the mass transfer of $\mathrm{H}^{+}$to the disk.

The most relevant assumption in this chapter is that the reactor dimension are large enough so as not to impact the mass transfer of $\mathrm{H}^{+}$to the surface of the disk.

Previous work studied the diffusion coefficient and the mass transfer rate in nonNewtonian fluids where viscosity is a function of shear rate (Hansford and Litt 1968; Mishra and Singh 1978; Rozieres et al. 1994). Hansford and Litt (1968) developed an equation to obtain the mass flux across a rotating disk in non-Newtonian fluids (powerlaw fluids):

$J_{m t}=\left[\frac{a^{\prime}}{3}\right]^{\frac{1}{3}} C_{b} D_{e}^{\frac{2}{3}}\left[\frac{1}{0.89}\right]\left[\frac{6 n^{\prime}+6}{5 n^{\prime}+7}\right]\left[\frac{K^{\prime}}{\rho}\right]^{\frac{-1}{3\left(\frac{1}{1+n^{\prime}}\right)}}\left[r^{\prime}\right]^{\frac{1}{3\left(\frac{1-n^{\prime}}{1+n^{\prime}}\right)}}[\omega]^{\frac{1}{1+n^{\prime}}}$

where

$a^{\prime}=a\left[1+\frac{1}{2}\left[\frac{1-n^{\prime}}{1+n^{\prime}}\right]\right]$

where $D_{e}$ is the diffusion coefficient, $a$ is the dimensionless radial velocity gradient at the disk surface, $n^{\prime}$ is the power-law index, $K^{\prime}$ is the power-law consistency index, $\rho$ is the fluid density, and $r$ ' is the radius of the disk. In their work, Hansford and Litt (1968) observed three flow regimes. These regimes are reverse flow, toroidal flow, and 
centrifugal flow. These flow regimes change as a function of shear rate, starting with reverse flow at the lowest shear and ending with centrifugal flow at the highest shear. It should be noted that none of the studies performed on non-Newtonian fluids accurately captured both the transport and surface reactions that take place during the RDA experiment. The hollow-core method reported by Gdanski and Norman (1986) is another tool used to study reaction kinetics. In the case of Non-Newtonian fluids, the hollow-core method does not require the fluid viscosity in calculating the rate of mass transfer. However, the rate of mass transfer calculation is dependent on position and utilizes an empirical approach to account for the effect of fluid leakoff on the observed acid flux.

Chang and Abbad (2011) simulated the fluid flow under a rotating disk using a commercial simulator. They showed that the flow is axisymmetric under the disk. However, they reported that the actual flow is more complex due to the time dependency of the fluid behavior in the corners of the reactor and close to the disk. Similar applications in the literature have discussed asymmetry in the velocity profile under a rotating disk (Duck 1986; Frueh and Read 1999; Vo et al. 2014; Vo et al. 2015). However, the apparatuses reported in the aforementioned works are not identical to the RDA. Duck (1986) studied the flow enclosed between two rotating disks and considered cases where one or both disks were rotating in the same and different directions. Duck's work assumed axisymmetric flow. 
Frueh and Read (1999) showed that a transition from axisymmetric flow to regular vortices occurs through a Hopf bifurcation. Frueh and Read (1999) also discussed transitions to modulated vortices, chaos, and highly irregular flow.

Lehmkuhl and Hudson (1971) investigated the impact of the reactor dimensions on the mass transfer of $\mathrm{H}^{+}$to the rotating disk where a stainless-steel disk and cinnamic acid were used. The disk thickness ranged from 0.38 to $1.02 \mathrm{~cm}$, and the diameter ranged from 5.59 to $14.22 \mathrm{~cm}$. Lehmkuhl and Hudson (1971) varied the ratio of the cylinder radius to the disk radius between 1.043 and 18.5, and the ratio between the axial gap, from the base of the disk to the bottom of the reactor, and the disk radius varied between 0.01 and 12.9. The tests were performed at rotational speeds ranging from 29 to $200 \mathrm{rpm}$. Lehmkuhl and Hudson (1971) concluded that the ratio between the cylinder radius and the disk radius does not affect the mass transfer of $\mathrm{H}^{+}$to the disk. These authors observed that only the axial distance between the base of the disk and the bottom of the reactor impact the mass transfer. However, in cases where $R_{\text {eh }}$ is larger than $50, \mathrm{H}^{+}$transfer to the disk is independent of the reactor dimensions. $R_{e h}$ is defined as:

$R_{e h}=\frac{\omega \mathrm{y}^{2}}{v}$

where $y$ is the axial distance between the base of the disk and the bottom of the reactor. To the best of the author's knowledge, the phenomenon of asymmetry in the RDA has not been studied yet. The effect of the reactor dimensions (diameter and axial gap) on the mass 
transfer of $\mathrm{H}^{+}$between calcite and hydrochloric acid $(\mathrm{HCl})$ has also not been investigated. As a result, this work aims to: (1) study flow regimes under the rotating disk for Newtonian and non-Newtonian fluids, (2) investigate the impact of the reactor boundaries on the mass transfer of $\mathrm{H}^{+}$to the disk in the RDA, and (3) determine dimensions of the reactor that minimize this impact.

\section{Turbulence in the Rotating Disk Apparatus}

In this chapter, I intentionally differentiate between the rotating-disk electrode (RDE) and the rotating-disk apparatus (RDA), although laboratory techniques for each are developed based on the theory of the mass transfer to a rotating disk (RD). The RDE is mainly used by electrochemists to study the processes associated with electron transfer. Generally, in RDE, (1) the metal alloy disks are highly polished until mirrorlike and are well centered, and (2) no considerable dissolution of disk or deposition of material on its surface occurs during the test. A discussion and the list of references are provided below.

Petroleum engineers use the RDA to study dissolution and corrosion processes. Contrary to studies involving the RDE, in most RDA studies, a substantial amount of a disk is dissolved during the experiment. Particularly, the RDA tests allow the determination of the acid-diffusion coefficient, a crucial parameter for designing a successful wellstimulation operation.

Levich (1962) derived Eq. 5 for the rate of mass transfer (j) to an RD as: 
$j=D^{2} / 3 v^{-1 / 6} \omega^{1 / 2} C_{b}$

where $D$ is the diffusion coefficient of the active species, $v$ is the kinematic viscosity of the fluid, $\omega$ is the angular velocity of the $\mathrm{RD}$, and $C_{b}$ is the concentration of the active species in the bulk of the solution. Eq. 5 was derived assuming:

- The fluid is Newtonian.

- The disk is sufficiently large so that edge effects are negligible.

- The laminar flow to the disk surface allows for uniform accessibility with an equal flux of the active species. As such, the concentration of the active species is a function only of the distance from the disk surface and is not a function of either radial or tangential position.

Later, Newman (1966) extended Levich's (1962) theory via the following equation:

$j=\frac{0.62048 S c^{-2 / 3}(v \omega)^{1 / 2}}{1+0.2980 S c^{-1 / 3}+0.1451 S c^{-2 / 3}}\left(C_{b}-C_{S}\right)$,

where $S c=v / D$ is the Schmidt number and $C_{S}$ is the concentration of the active species on the surface of the disk.

In order to calculate the diffusion coefficient using Eq. 6, the RD experiments should be performed in a mass-transfer limited regime such that the assumption of laminar flow to the disk surface is satisfied. The Reynolds number used to predict the flow regime to an $\mathrm{RD}$ is calculated using the following equation:

$R e=\frac{\omega r^{2}}{v}$, 
where $r$ is the radial distance from the center of rotation. The flow regime's transition from laminar to turbulent starts at a critical Reynolds number (hereafter $\mathrm{Re}_{\mathrm{cr}}$ ). Levich (1962) stated that the laminar flow was ordinarily sustained up to a $\operatorname{Re}_{\mathrm{cr}} \approx 10^{4}$, but with very well-polished and centered disks, the laminar flow was sustained up to a $\operatorname{Re}_{\mathrm{cr}} \approx 10^{5}$. Readers are referred to literature on fluid dynamics reporting the experimental and theoretical works on laminar-turbulent flow transition in RD boundary layer (Gregory et al. 1955; Kreith et al. 1959; Clarkson et al. 1980; Kobayashi et al. 1980; Malik et al. 1981; Kohama 1984; Wilkinson and Malik 1985; Malik 1986; Balachandar et al. 1992; Kohama and Sudaf 1992; Aubry 1998; Jarre et al. 1996; Lingwood 1996; Corke et al. 2007; Harris et al. 2012).

The theory of an RD was first applied to study the electrochemical processes using an RDE (Daguenet 1968; Ellison et al. 1971; Chin and Litt 1972; Nanis and Klein 1972; Vahdat and Newman 1973; Bruckenstein and Miller 1977; Mohr and Newman 1976; Albery and Bruckenstein 1983; Dong et al. 2008), and was later adopted to analyze the chemical dissolution reactions using RDA (Boomer et al. 1972; Lund et al. 1973, Lund et al. 1975; Fredd and Fogler 1998; Taylor et al. 2004; Alkhaldi et al. 2010; Rabie et al. 2014; Khalid et al. 2015; Aldakkan et al. 2018; Hall-Thompson et al. 2020; Sayed et al. 2020; Kotb et al. 2021). 
Although the early $\mathrm{RDE}$ studies reported a $\mathrm{Re}_{\mathrm{cr}}=1.7-3 \times 10^{5}$, the designers of the first RDA (Boomer et al. 1972) cite Levich's (1962) work and mention that for the flow in the vicinity of the disk to be laminar, the Reynolds number must not exceed $10^{4}-10^{5}$. Nevertheless, $\operatorname{Re}_{\mathrm{cr}}=3 \times 10^{5}$ was assumed in the following RDA studies. With such a high $\mathrm{Re}_{\mathrm{cr}}$ value, for 1.0 and 1.5 in. diameter disks rotating in Newtonian fluid of kinematic viscosity around $0.01 \mathrm{~cm}^{2} / \mathrm{s}$, the laminar flow should exist until the disk rotational speeds reach approximately 11,800 and 5,300 rpm, respectively. These values are well above 2,000 $\mathrm{rpm}$, the maximum disk-rotational speed most commercial RDAs are able to maintain. Thus, all published RDA studies to date were assumed to be performed in the laminar regime.

No study has been done to analyze the adequacy of assuming $\operatorname{Re}_{\mathrm{cr}}=3 \times 10^{5}$ in RDA setups. Unlike most electrochemical and fluid dynamics studies, the RDA experiments result in considerable dissolution of the RD. Also, there is no information on how well centered the disks are in commercial RDA configurations. Both eccentricity and dissolution of the disk can decrease the $\mathrm{Re}_{\mathrm{cr}}$. For $1.5 \mathrm{in}$. diameter disk rotating at 2,000 rpm in $\mathrm{HCl}$ solution with a kinematic viscosity of $0.0114 \mathrm{~cm}^{2} / \mathrm{s}$, the Reynolds number is $6.7 \times 10^{4}$. This value is within the range proposed by Levich (1962).

Running the RDA tests at transition or turbulent flow regimes increases the mass transfer to the surface of the RD and results in overestimation of the acid-diffusion coefficient. 
This work aims to (1) validate laminar flow assumption at typical experimental conditions, and (2) optimize the acid-diffusion coefficient measurements.

\section{Computational Fluid Dynamics Model for Simulating the Rotating Disk Apparatus}

Acidizing is a well-stimulation procedure whereby acid is injected downhole below the fracture pressure to improve oil and gas productivity. The objective of acidizing varies according to the formation type. In the case of sandstone reservoirs, the objective is to minimize formation damage, while in the case of carbonate reservoirs, the objective is to surpass formation damage and create high permeability channels known as wormholes. To fully realize these objectives, a clear understanding of (a) damage type, (b) formation temperature, (c) fluid properties in the reservoir, and (d) the fundamentals of the underlying reaction kinetics at different conditions is essential.

The rotating disk apparatus (RDA) has been typically used for studying reaction kinetics between different rocks and acid systems (Boomer et al. 1972; Lund et al. 1973a; Roberts and Guin 1975; Fredd and Fogler 1998; Conway et al. 1999; Taylor and Nasr-El-Din 2009; Rabie et al. 2014; Li et al. 2016; Liao et al. 2017; Abdelgawad et al. 2018; Kotb and NasrEl-Din 2020; Sayed et al. 2020; Ivanshin et al. 2021). In the seminal work by Levich (1942), the author studied the rate of mass transfer of $\mathrm{H}^{+}$in Newtonian fluids to a rotating disk in a reactor and developed the following equation to calculate it:

$\frac{i}{N F}=\frac{D_{e}}{1-t_{r}} \frac{d C_{b}}{d y}=\frac{\frac{D_{e}\left(C_{b}-C_{s}\right)}{\left(1-t_{r}\right)}}{\int_{0}^{\infty} \exp \left\{\int_{0}^{y V y} \frac{D_{e}}{D_{e}} d y\right\} d y}$ 
where

$$
V_{y}=\sqrt{\omega v}\left[-0.51023 \frac{\omega}{v} y^{2}+\frac{1}{3}\left(\frac{\omega}{v}\right)^{1.5} y^{3}-0.10265\left(\frac{\omega}{v}\right)^{2} y^{4}+\cdots\right],
$$

$i$ is the electric current density, $N$ is the number of electrons produced upon the reaction of one reactant ion or molecule, $F$ is Faraday's constant, $t_{r}$ is the ion transport number, $D_{e}$ is the diffusion coefficient of $\mathrm{H}^{+}, C_{b}$ is the bulk concentration of the reactant, $C_{s}$ is the reactant concentration on the disk surface, $\omega$ is the disk rotational speed, $v$ is the kinematic viscosity of the fluid, $V_{y}$ is the acid velocity in the axial direction, and $y$ is the axial distance from the disk to the point of the measurment of $V_{y}$.

Since it was difficult to find a closed-form analytical solution for Eq. 8, the author only considered the first term in Eq. 9 for Schmidt numbers higher than 100 and obtained:

$\frac{i\left(1-t_{r}\right)}{N F}=J_{m t}=0.6205 S_{c}^{-\frac{2}{3}}(v \omega)^{0.5}\left(C_{b}-C_{s}\right)$

where $J_{m t}$ is the rate of mass transfer of $\mathrm{H}^{+}$to the rotating disk, and $S_{c}$ is the Schmidt number. Schmidt number is defined as the ratio between the kinematic viscosity and the diffusion coefficient of a fluid. Levich's (1942) equation was then numerically solved by Gregory and Riddiford (1956), and the results were fitted empirically through the following expression: 
$J_{m t}=\frac{0.554 S_{c}^{-\frac{2}{3}}(v \omega)^{0.5}\left(C_{b}-C_{s}\right)}{0.8934+0.316 S_{c}^{-0.36}}$

Newman (1966) later expanded the exponential function in Eq. 8 for Schmidt numbers larger than 100 and solved it analytically to obtain:

$J_{m t}=\frac{0.62048 S_{c}^{-\frac{2}{3}}(v \omega)^{0.5}}{1+0.298 S_{c}^{-\frac{1}{3}}+0.1451 S_{c}^{-\frac{2}{3}}}\left(C_{b}-C_{S}\right)$

Newman (1966) compared the numerical solution of Eq. 8, which is still subject to error, with Eqs. 10 to 12. He reported that for fluids with a Schmidt number higher than 100, only Eqs. 11 and 12 yielded a relative error less than 5\% when compared to the numerical solution of Eq. 8.

The equation developed by Newman (Eq. 12) has since been used to estimate the diffusion coefficient for Newtonian fluids in the RDA. It is worth mentioning that the equation is based on the following assumptions:

- The surface area of the disk and fluid properties (acid concentration, density, and viscosity) remain the same throughout the experiment.

- The flow is single phase and laminar. Laminar flow is defined by a Reynolds number lower than $10^{5}$ (Levich 1962).

- The reactor boundaries are infinite so as not to impact the mass transfer of $\mathrm{H}^{+}$ to the disk. 
- The Schmidt number is higher than 100.

Kotb and Nasr-El-Din (2020) studied the impact of these assumptions on the mass transfer of $\mathrm{H}^{+}$to the disk and the diffusion coefficient calculations. They showed that some of these assumptions in Newman's equation are not met under practical scenarios and can lead to inaccuracies in calculating the acid diffusion coefficient. In fact, the equation has not been updated or revisited since its development in 1966. Consequently, there are challenges posed when performing carbonate formation acidizing and acid fracturing, both of which depend on an accurate estimation of the diffusion coefficient (Roberts and Guin 1974; Economides and Nolte 1989).

To obtain the mass transfer rate of $\mathrm{H}^{+}$to the disk, Lund et al. (1973b) described the relationship between the reaction rate coefficient and the rate of mass transfer of hydrochloric acid $(\mathrm{HCl})$ to a rotating disk as:

$J_{m t}=\left[k C_{s}^{n}\right]$

where $k$ is the reaction rate coefficient and $n$ is the reaction order. However, the reaction of $\mathrm{HCl}$ with carbonates is assumed to be mass transfer limited. As a result, $C_{s}$ is assumed to be zero, which makes it difficult to estimate the reaction rate coefficient. Lund et al. (1975) calculated a value for $k=1.34 \times 10^{-6} \mathrm{~cm} / \mathrm{s}$ at $-4^{\circ} \mathrm{F}$, where the reaction of $\mathrm{HCl}$ with carbonates is reaction limited. Unfortunately, this is not a practical temperature for 
operational purposes, which necessitates the need for a method to estimate the reaction rate coefficient at temperatures higher than $150^{\circ} \mathrm{F}$.

One method used for obtaining the reaction rate coefficient is the Arrhenius equation (McNaught and Alan 1997), which requires the activation energy of the reaction. However, there is no agreement in the literature on the correct value of activation energy for the reaction of $\mathrm{HCl}$ with carbonate rocks. The value in the literature varies between 20 and 60 KJ/mol (Lund et al. 1975; Sjöberg 1976; Sjöberg and Rickard 1984; Arakaki and Mucci 1995; Gutjahr et al. 1996; Gledhill and Morse 2006; Finneran and Morse 2009; Peng et al. 2015). This wide range is an outcome of different conditions and methods for calculation. Lund et al. (1975) used the RDA at $77^{\circ} \mathrm{F}$ to obtain an activation energy value for carbonate dissolution of $60 \mathrm{KJ} / \mathrm{mol}$. According to Sjöberg (1976), work was performed at a $\mathrm{pH}$ of $8-10$ and temperatures between 41 and $122^{\circ} \mathrm{F}$ for carbonate dissolution and obtained $35 \mathrm{KJ} / \mathrm{mol}$ for the activation energy. Sjöberg and Rickard (1984) later studied the activation energy for carbonate dissolution at $8.4 \mathrm{pH}$ at temperatures between 34 and $143^{\circ} \mathrm{F}$ and obtained an activation energy of $46 \pm 4 \mathrm{KJ} / \mathrm{mol}$. Gutjahr et al. (1996) used a stirred vessel at neutral to alkaline $\mathrm{pH}$ to obtain a $35 \mathrm{KJ} / \mathrm{mol}$ activation energy value. Gledhill and Morse (2006) and Finneran and Morse (2009) studied crushed samples at a pH larger than 5.4 and reported an activation energy of around $20 \mathrm{KJ} / \mathrm{mol}$.

This lack of consensus dictates the need to develop a new method for accurately estimating the diffusion coefficient and the reaction rate coefficient from the RDA. Understanding 
the mechanisms of acid-rock interaction is essential to performing a successful formation acidizing procedure in the field. The objectives of the current work are to: (a) develop a calibrated computational fluid dynamics (CFD) model and a proxy model that simulate the reaction between $\mathrm{HCl}$ and carbonate rocks in the RDA and (b) use these models to estimate, for the first time, the diffusion coefficient and the reaction rate coefficient of the reaction in the RDA, which takes into account the impact of changing porosity of the rock, changing the concentration of the acid and the impact of the container boundaries. 


\title{
CHAPTER II
}

NEW INSIGHTS INTO MASS TRANSFER WHEN USING THE ROTATING DISK APPARATUS FOR NEWTONIAN AND NON-NEWTONIAN FLUIDS*

\author{
Method \\ Experimental Studies
}

In order to study flow regimes under a rotating disk, a non-Newtonian fluid was prepared by using an anionic polyacrylamide-based friction reducer. Four solutions were prepared with concentrations ranging from 0.05 to $0.4 \mathrm{wt} \%$. Viscosity for these solutions was measured using an M3600 viscometer at shear rates ranging from 0.01 to $1,000 \mathrm{~s}^{-1}$ at room pressure and temperature. The solution was then added to a glass beaker, and a Hastelloy disk was rotated in the solution using an overhead blender at room temperature and pressure. A petroleum-based color was added to the solution to visualize the flow under the disk. The rotational speeds tested ranged from 50 to1,800 rpm.

Three RDA experiments were performed. Fig. II-1 shows the schematic of the RDA used (Rabie et al. 2014; Kotb et al. 2018). The reservoir and the reactor have $550 \mathrm{~cm}^{3}$ of volume with $450 \mathrm{~cm}^{3}$ of acid used. The diameter of the reactor is $8 \mathrm{~cm}$, the disk diameter is 3.8 $\mathrm{cm}$, the disk thickness is $1.3 \mathrm{~cm}$, and the axial distance between the base of the disk and the bottom of the reactor is $3.2 \mathrm{~cm}$. In the experiments, $15 \mathrm{wt} \% \mathrm{HCl}$ with $1 \mathrm{wt} \%$ corrosion

\footnotetext{
* Part of this chapter is reprinted with permission from "New Insights into Mass Transfer When Using the Rotating Disk Apparatus for Newtonian and Non-Newtonian Fluids" by Kotb, A. and Nasr-El-Din, H. A., 2020, SPE J, Copyright 2020 by Society of Petroleum Engineers.
} 
inhibitor was used at $150^{\circ} \mathrm{F}$ at rotational speeds of 132,584 , and $1,172 \mathrm{rpm}$. The procedure outlined in Fredd (1997) was followed for running tests. A marble disk with 1.5 in. diameter and 0.5 in. thickness was heated to $220^{\circ} \mathrm{F}$ to evaporate water and was then weighed. Next, sandpaper was used to smooth the sides of the disk. The disk was further soaked in $0.1 \mathrm{M} \mathrm{HCl}$ for 30 minutes to remove fines and then cleaned with deionized water. It was then laminated with shrinkable Teflon was applied such that only the base of the disk was exposed to the acid. The disk was then placed in the reactor and was connected to a magnetic drive for rotation. $\mathrm{HCl}$ acid $\left(450 \mathrm{~cm}^{3}\right.$ volume $)$ was heated and pressurized to $1,500 \mathrm{psi}$ in the reservoir. The acid was then transported to the reactor - a process that takes 10-12 s after which the reservoir was pressurized to $1,500 \mathrm{psi}$, and disk rotation started. The pressure keeps $\mathrm{CO}_{2}$ in solution and maintains a single-phase fluid (Welton and Van Domelen 2008; Nasr-El-Din et al. 2008; Taylor and Nasr-El-Din 2009). Acid samples were taken from the reactor at 1-minute intervals over the first 5-minute test duration. Calcium concentration in these samples was measured using inductively coupled plasma (ICP). This concentration, coupled with the initial porosity and surface area of the disk that is exposed to the acid, was used to calculate the dissolution rate. 


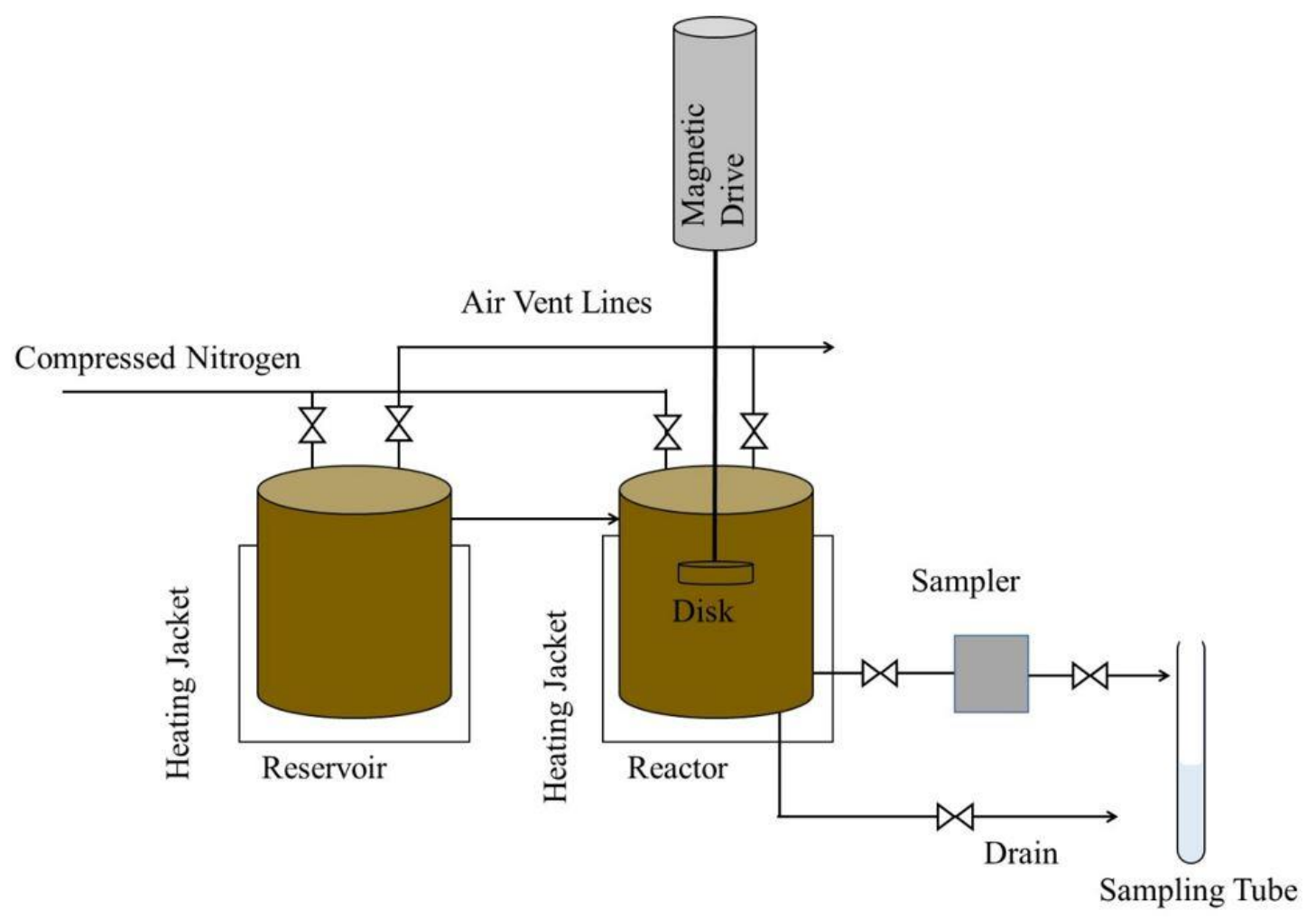

Fig. II-1 A schematic diagram for the rotating disk. Reprinted with permission from (Kotb and Nasr-El-Din 2020).

\section{Simulation Studies}

Five reactors were simulated (Table II-1) to study the impact of the reactor dimensions on the velocity profile and the mass transfer of $\mathrm{H}^{+}$to the disk. Reactor A's dimensions match the reactor used in the experiments. 


\begin{tabular}{cccccc} 
Case & $\begin{array}{c}\text { Reactor } \\
\text { Diameter } \\
(\mathrm{cm})\end{array}$ & $\begin{array}{c}\text { Reactor } \\
\text { Height }(\mathrm{cm})\end{array}$ & $\begin{array}{c}\text { Axial Distance } \\
\text { Between the Disk } \\
\text { and the Bottom } \\
\text { Reactor Boundary } \\
(\mathrm{cm})\end{array}$ & $\begin{array}{c}\text { Reactor } \\
\text { Volume } \\
\left(\mathrm{cm}^{3}\right)\end{array}$ & $\begin{array}{c}\text { Number of } \\
\text { Cells } \\
\text { (Million) }\end{array}$ \\
\hline A & 8 & 9.3 & 3.2 & 450 & 1.2 \\
B & 11.4 & 9.3 & 3.2 & 950 & 1.4 \\
C & 8 & 13.4 & 7.3 & 673 & 2.1 \\
D & 11.4 & 13.4 & 7.3 & 1367 & 5.3 \\
E & 15.2 & 17.8 & 11.7 & 3229 & 7.1 \\
\hline
\end{tabular}

Table II-1 Dimensions of the simulated reactors and the number of cells. Experiments were performed in Reactor A. Reprinted with permission from (Kotb and Nasr-El-Din 2020).

In order to simulate the reaction in the RDA, the two-scale (averaged-continuum) approach was used. This model has been used previously by Zhang et al. (2014) and Ali and Nasr-El-Din (2019). The calculations in the model are divided into Darcy-scale equations presented by Navier-Stokes and pore-scale equations. The commercial software ANSYS Fluent was used to solve the mass-continuity, momentum, and transport equations using the finite volume method. The conservation of mass equation is written as:

$\frac{\partial \rho}{\partial t}+\nabla \cdot(\rho \boldsymbol{u})=S_{m}$

where $t$ is time, $\boldsymbol{u}$ is the velocity vector, and $S_{m}$ is the source mass added to the continuity equation. The conservation of momentum is expressed as:

$\frac{\partial(\rho u)}{\partial t}+\nabla \cdot(\rho \boldsymbol{u} \boldsymbol{u})=-\nabla p+\nabla \cdot(\tau)+\rho g+f, \ldots$ 
where $p$ is pressure, $\tau$ is the stress tensor, $\rho g$ is the gravitational body force, and $f$ is the sum of external body forces. The transport equations are user-defined codes in the computational fluid dynamics (CFD) software that use $\mathrm{C}$ programming language. The workflow of the equations used to solve the model is discussed in Ali et al. (2019).

Simulation results are a function of mesh size and type. To keep results consistent, all developed models used the tetrahedron elements. Tetrahedron elements were selected because they allow results closer to theoretical ones over hexahedral elements (Ramos and Simoes 2006), and the CPU time in both methods is similar (Cifuentes and Kalbag 1992). Skewness, orthogonality, and aspect ratio had to be optimized to maintain mesh stability, prevent divergence, and minimize computational time.

In order to determine the optimum number of cells for each reactor, the five reactors were run under the same conditions discussed in Table II-2 at 1,000 rpm with varying the number of cells. Once the relative change in the amount of rock dissolved dropped below $5 \%$, that mesh was selected. This threshold was chosen because a relative difference that low can be attributed to computational error and can be neglected. Fig. II-2 shows the impact of changing the number of cells on the disk dissolved. Table II-1 includes the number of cells for each reactor used in this study. The rate of mass transfer of $\mathrm{Ca}^{+2}$ was then calculated using the following equation: 
$J_{m t}=\frac{C_{r} \alpha^{\prime} \omega^{0.5}}{t A_{d}}$

where $C_{r}$ is the total calcium ion concentration in the disk, $\alpha^{\prime}$ is the amount of rock dissolved, $t$ is the test duration, and $A_{d}$ is the initial area of the disk exposed to the acid calculated using:

$A_{d}=\pi\left(r^{\prime}\right)^{2}$

where $r$ ' is the radius of the disk. The porosity is ignored in Eq. 4 because marble has 0 porosity. The results were then used to study the impact of the reactor dimensions on the mass transfer of $\mathrm{H}^{+}$to the disk. 
Test Purpose

\begin{tabular}{cccc}
\hline Parameters & $\begin{array}{c}\text { Mass Transfer } \\
\text { Rate and } \\
\text { Maximum Radial } \\
\text { Velocity }\end{array}$ & $\begin{array}{c}\text { Flow } \\
\text { Asymmetry }\end{array}$ & $\begin{array}{c}\text { Matching } \\
\text { Arslan et al. } \\
(2017)\end{array}$ \\
\hline Acid concentration, wt\% & 15 & $15-36$ & 18.76 \\
Disk rotational speed, rpm & $10-1,000$ & $100-1,000$ & $200-1,200$ \\
Temperature, ${ }^{\circ} \mathrm{F}$ & 150 & $70-150$ & 100 \\
Acid density, $\mathrm{g} / \mathrm{cm}^{3}$ & 1.06 & & 1.075 \\
Acid viscosity, $\mathrm{cp}$ & 0.5 & 0.89 \\
Diffusion coefficient, $\mathrm{m}^{2} / \mathrm{s}$ & $3.34 \mathrm{E}-09$ & $3.13 \mathrm{E}-09$ \\
Reaction rate coefficient, & 0.3 & 0.077 \\
$\mathrm{~m} / \mathrm{s}$ & 0.1 & 0.1 \\
Initial porosity, vol\% & 5 & 5 \\
Test duration, $\mathrm{min}$ & 5 & & 5 \\
\hline
\end{tabular}

Table II-2 Parameters for the simulation run for the different tests performed in this work. For the mass transfer rate and maximum radial velocity, the acid density was measured using a density meter. The acid viscosity was obtained from

Nishikata et al. (1981), the diffusion coefficient was obtained from Qiu et al. (2015), and the reaction rate coefficient was obtained from Peng et al. (2015). For flow asymmetry, fluid density and viscosity were obtained from Nishikata et al. (1981).

For Matching Arslan et al. (2017), the temperature, acid concentration, acid density, acid viscosity, and diffusion coefficient were reported by Arslan et al. (2017). The reaction rate coefficient was calculated based on the activation energy reported by Arslan et al. (2017) and the reaction rate constant reported by Peng et al. (2015). Reprinted with permission from (Kotb and Nasr-El-Din 2020). 


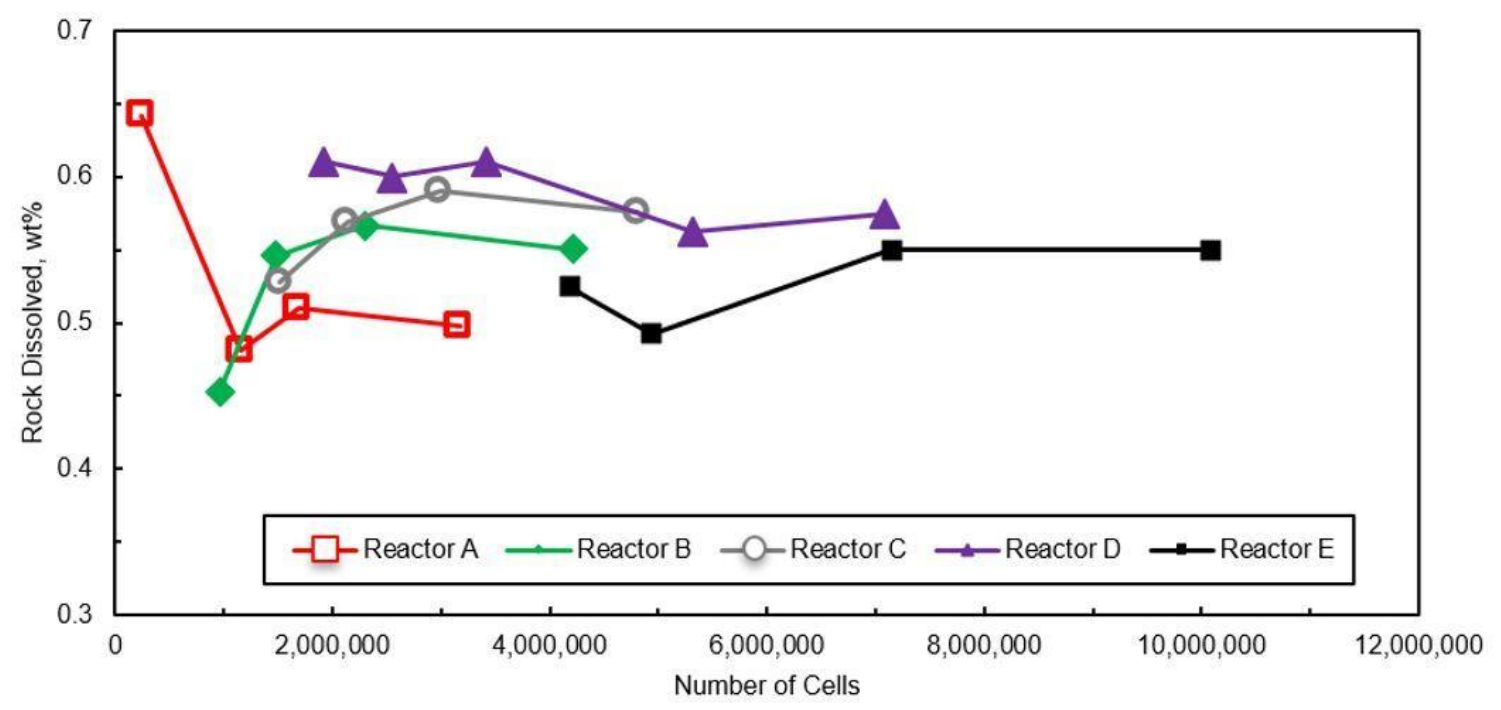

Fig. II-2 Effect of the number of cells in the five reactors simulated on the rock dissolved for five minutes. Reprinted with permission from (Kotb and Nasr-El-Din 2020).

After the five reactors were modeled, the simulation results were compared to the experimental results. Finally, the model was validated by comparing the models' performance against the results obtained by Arslan et al. (2017). Arslan et el. (2017) performed four RDA experiments at $100^{\circ} \mathrm{F}$ using $18.76 \mathrm{wt} \% \mathrm{HCl}$ and $1 \mathrm{wt} \%$ corrosion inhibitor at 200,600,800, and 1,200 rpm.

\section{Results and Discussion}

\section{Non-Newtonian Fluid Properties and Velocity Profile}

Fig. II-3 shows the viscosity profile for the non-Newtonian fluid at different polymer concentrations. Viscosity ranges between $19,000 \mathrm{cp}$ for the $0.4 \mathrm{wt} \%$ at $0.1 \mathrm{~s}^{-1}$ shear rate and $11 \mathrm{cp}$ in the case of $0.05 \mathrm{wt} \%$ at $1,000 \mathrm{~s}^{-1}$ shear rate. A Hastelloy disk attached to an overhead mixer was placed in each of the four solutions. In the case of 0.4 and $0.2 \mathrm{wt} \%$, 
the disk did not create enough shear to cause significant motion under the disk. However, in the case of 0.1 and $0.05 \mathrm{wt} \%$, the fluid showed reverse flow for rotational speeds up to 1,800 rpm (Fig. II-4).

Impact of Reactor Dimensions on the Fluid Flow in Newtonian Fluids

Levich (1962) reported that the flow below a rotating disk is axisymmetric. The reactors in Table II-1 were run at different conditions discussed in Table II-2 to investigate the findings of Levich (1962). Fig. II-5 to Fig. II-9 show the impact of reactor dimensions, disk rotational speed, and viscosity on the velocity profile in the RDA. Only case A with $2 \mathrm{cp}$ at $100 \mathrm{rpm}\left(36 \mathrm{wt} \% \mathrm{HCl}\right.$ at $70^{\circ} \mathrm{F}$ Nishikata et al. 1981) showed axisymmetric flow. Other conditions showed a wide variety of non-axisymmetric velocity profiles. This asymmetry agrees with the literature in similar applications (Frueh and Read 1999; Vo et al. 2014; Vo et al. 2015). From the results, it can be concluded that a transition from axisymmetric flow can be observed for $\mathrm{R}_{\mathrm{ehr}}$ larger than 8,000 . $\mathrm{R}_{\mathrm{ehr}}$ is defined as:

$R_{e h r}=\frac{\omega \mathrm{yr}_{r}}{v}$

where $r_{r}$ is the radius of the reactor, $\omega$ is the disk rotational speed, and $y$ is the axial distance between the disk and the reactor boundary. Table II-3 summarizes the flow regimes and the $\mathrm{R}_{\mathrm{ehr}}$ values for the different simulations performed in this work. 


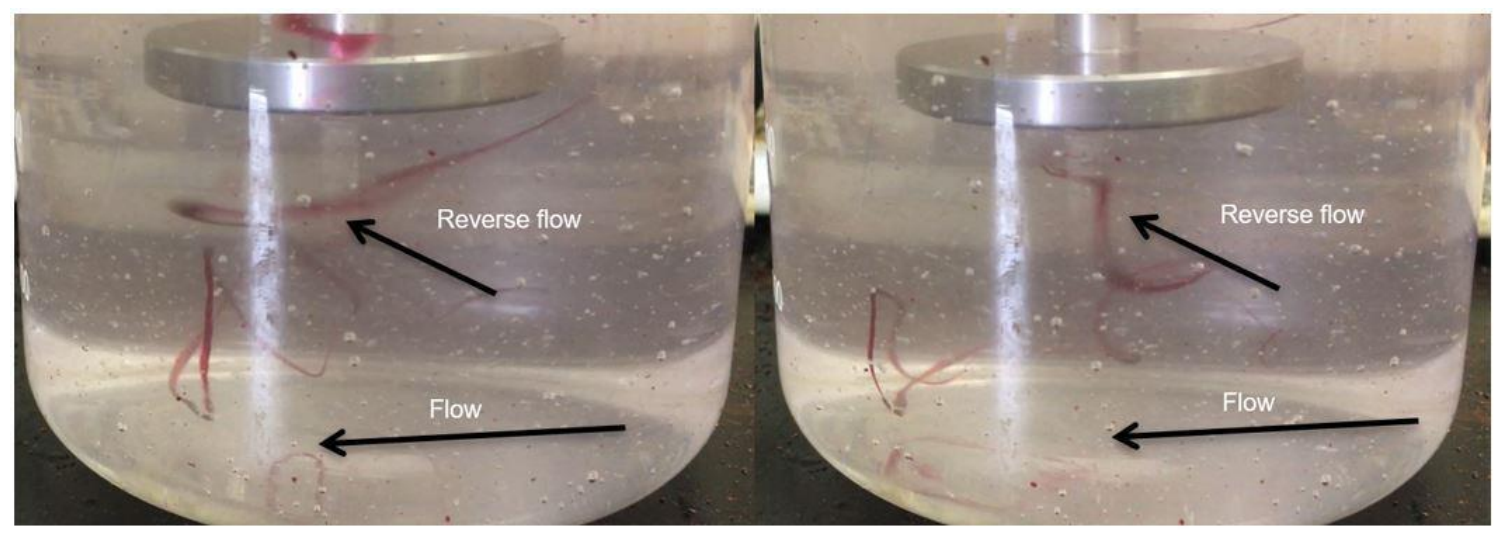

Fig. II-3 Effect of polymer concentration on the viscosity of polymer solutions. Reprinted with permission from (Kotb and Nasr-El-Din 2020).

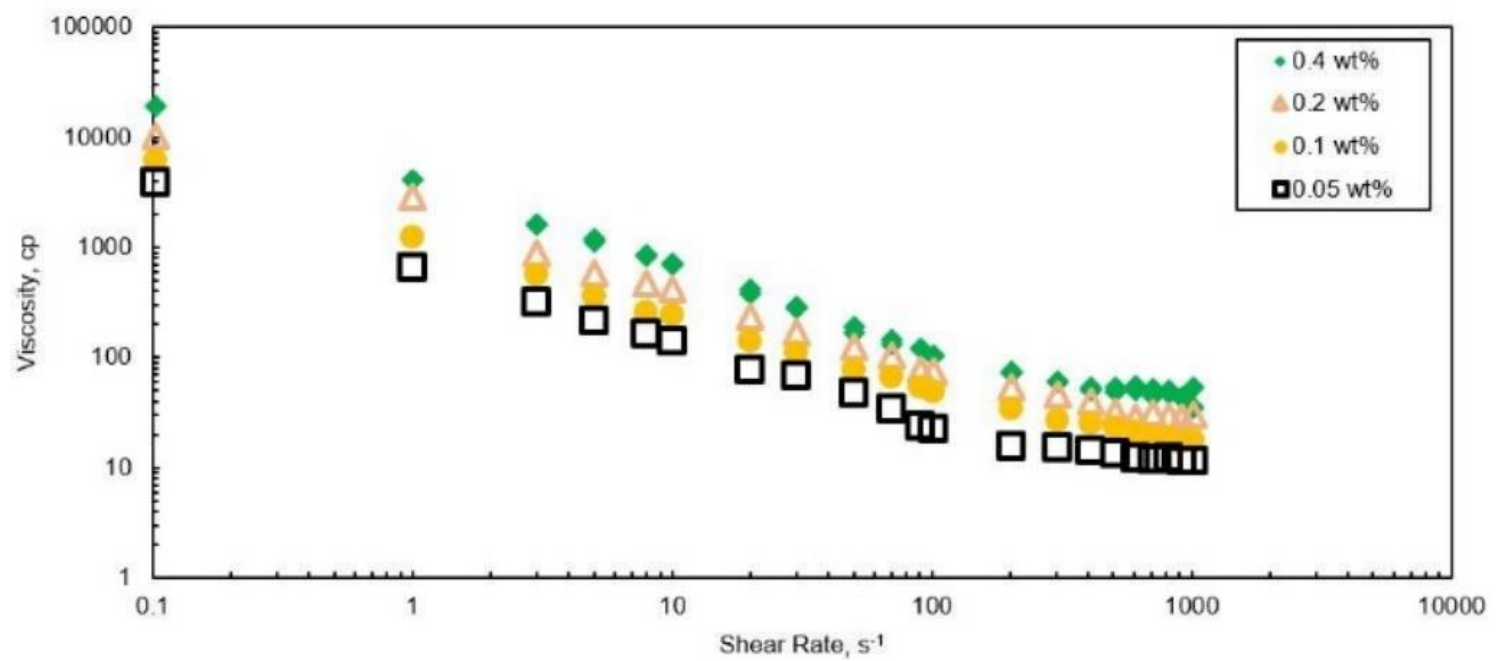

Fig. II-4 Petroleum-based dye (red) showing reverse flow under the disk for 0.1 wt \% polymer solution at $1,800 \mathrm{rpm}$. Reprinted with permission from (Kotb and Nasr-El-Din 2020). 

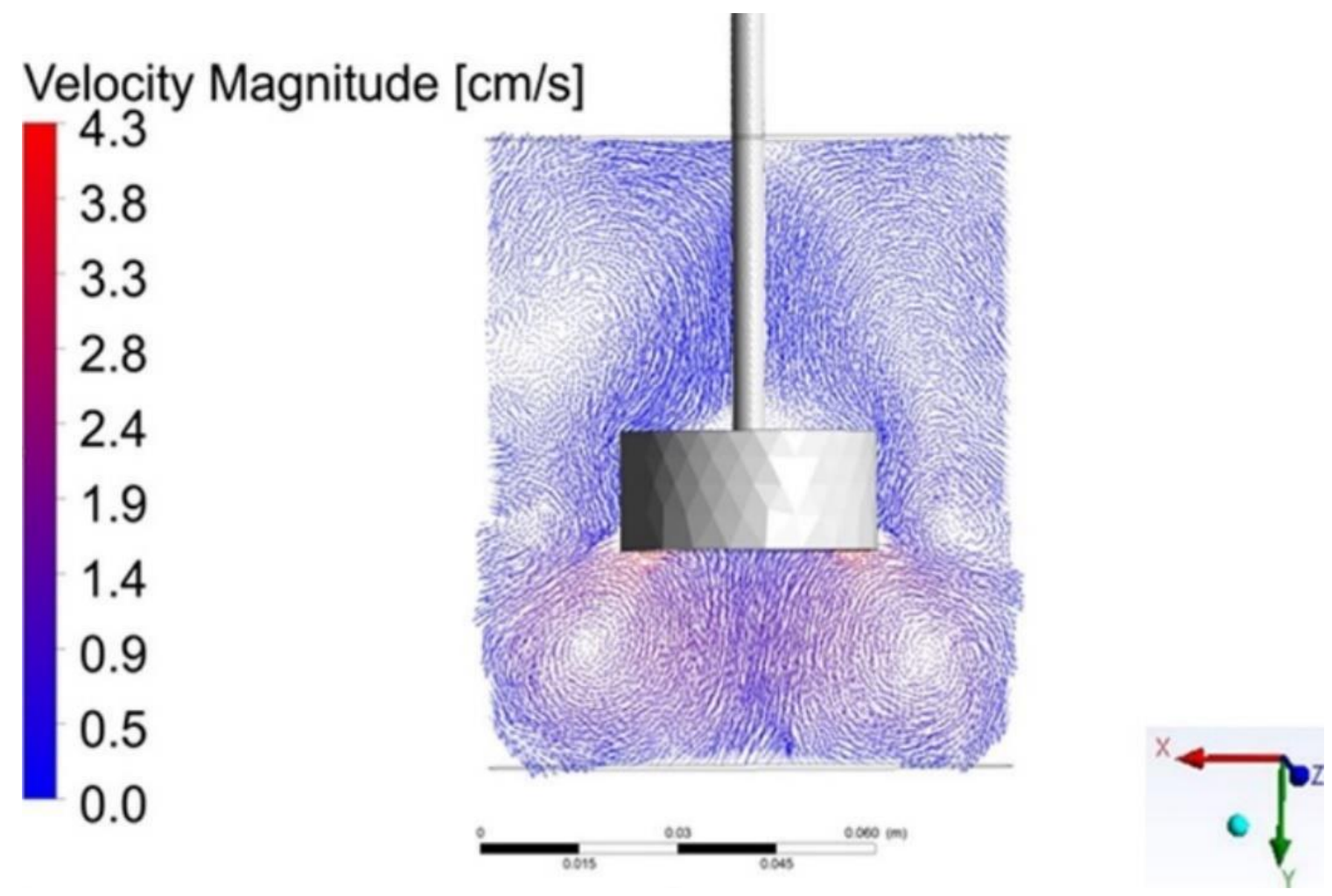

Fig. II-5 Velocity profile for reactor A with 2 cp HCl $\left(36\right.$ wt \% at $70^{\circ} \mathrm{F}$ Nishikata et al. 1981) at $300 \mathrm{~s}$ with $100 \mathrm{rpm}$. Reactor height is $9.3 \mathrm{~cm}$ and reactor diameter is 8 $\mathrm{cm}$. Axial distance between the base of the disk and the bottom of the reactor is 3.2 cm. Axisymmetric flow is observed. Reprinted with permission from (Kotb and Nasr-El-Din 2020). 

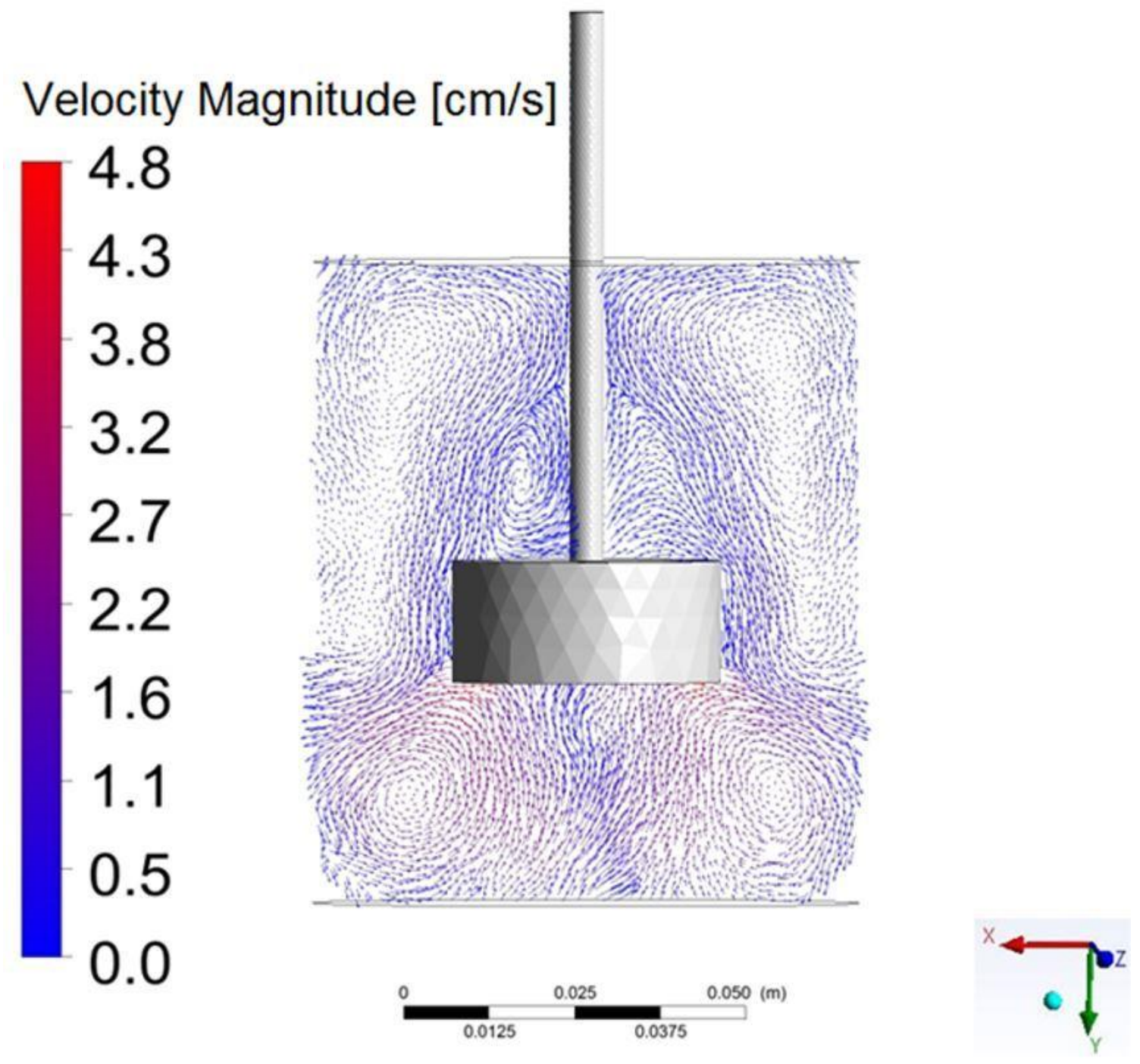

Fig. II-6 Velocity profile for reactor A with $1 \mathrm{cp} \mathrm{HCl} \mathrm{(15} \mathrm{wt \%} \mathrm{at} \mathrm{86} \mathrm{F}$ Nishikata et al. 1981) at $300 \mathrm{~s}$ with $100 \mathrm{rpm}$. Reactor height is $9.3 \mathrm{~cm}$ and reactor diameter is 8 $\mathrm{cm}$. Axial distance between the base of the disk and the bottom of the reactor is 3.2 cm. Transition from axisymmetric flow is observed. Reprinted with permission from (Kotb and Nasr-El-Din 2020). 


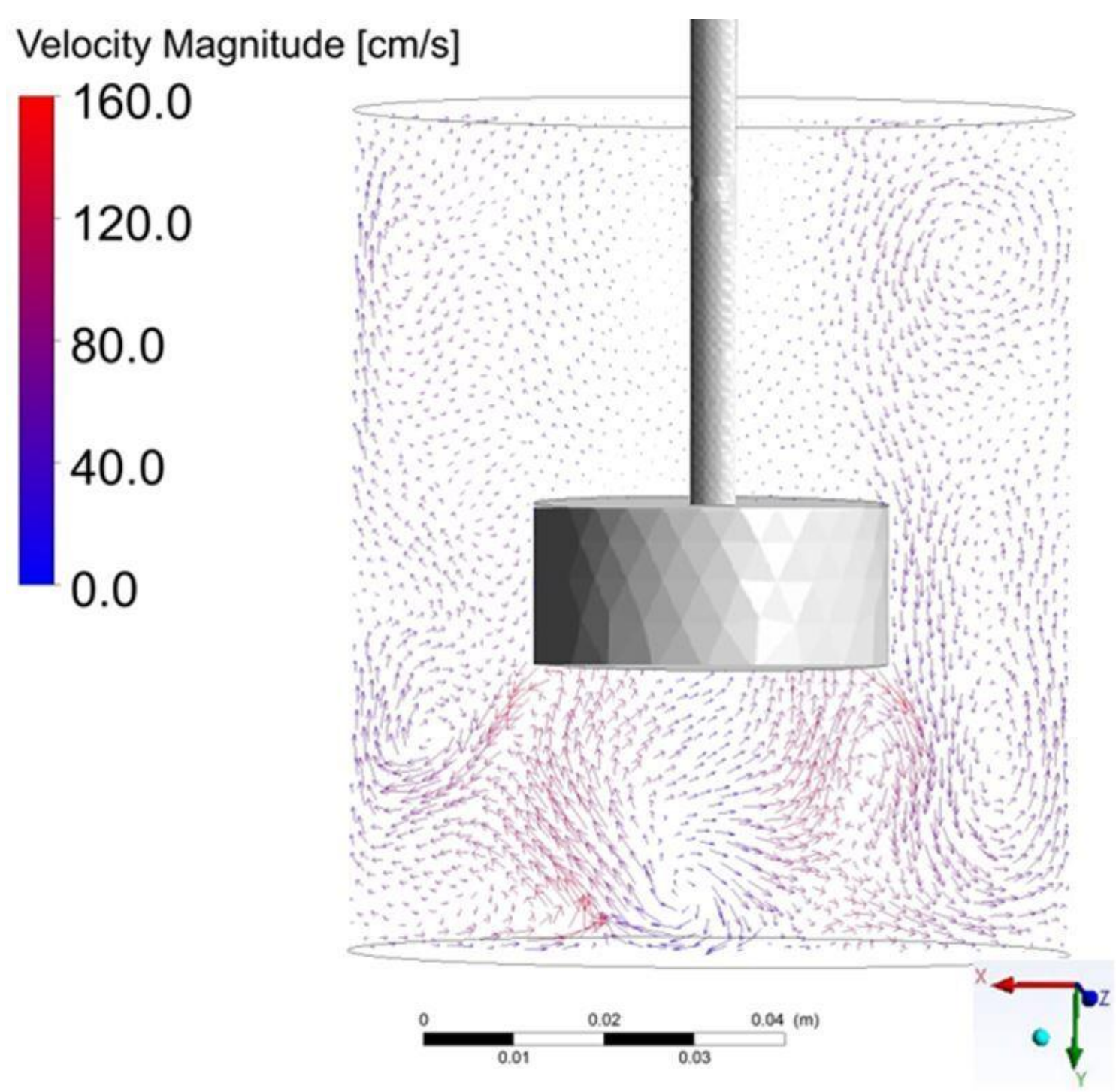

Fig. II-7 Velocity profile for reactor A with $0.5 \mathrm{cp} \mathrm{HCl}\left(15 \mathrm{wt} \%\right.$ at $150^{\circ} \mathrm{F}$ Nishikata et al. 1981) at $300 \mathrm{~s}$ with 1,000 rpm. Reactor height is $9.3 \mathrm{~cm}$ and reactor diameter is $8 \mathrm{~cm}$. Axial distance between the base of the disk and the bottom of the reactor is $3.2 \mathrm{~cm}$. Asymmetric flow is observed. Reprinted with permission from (Kotb and Nasr-El-Din 2020). 


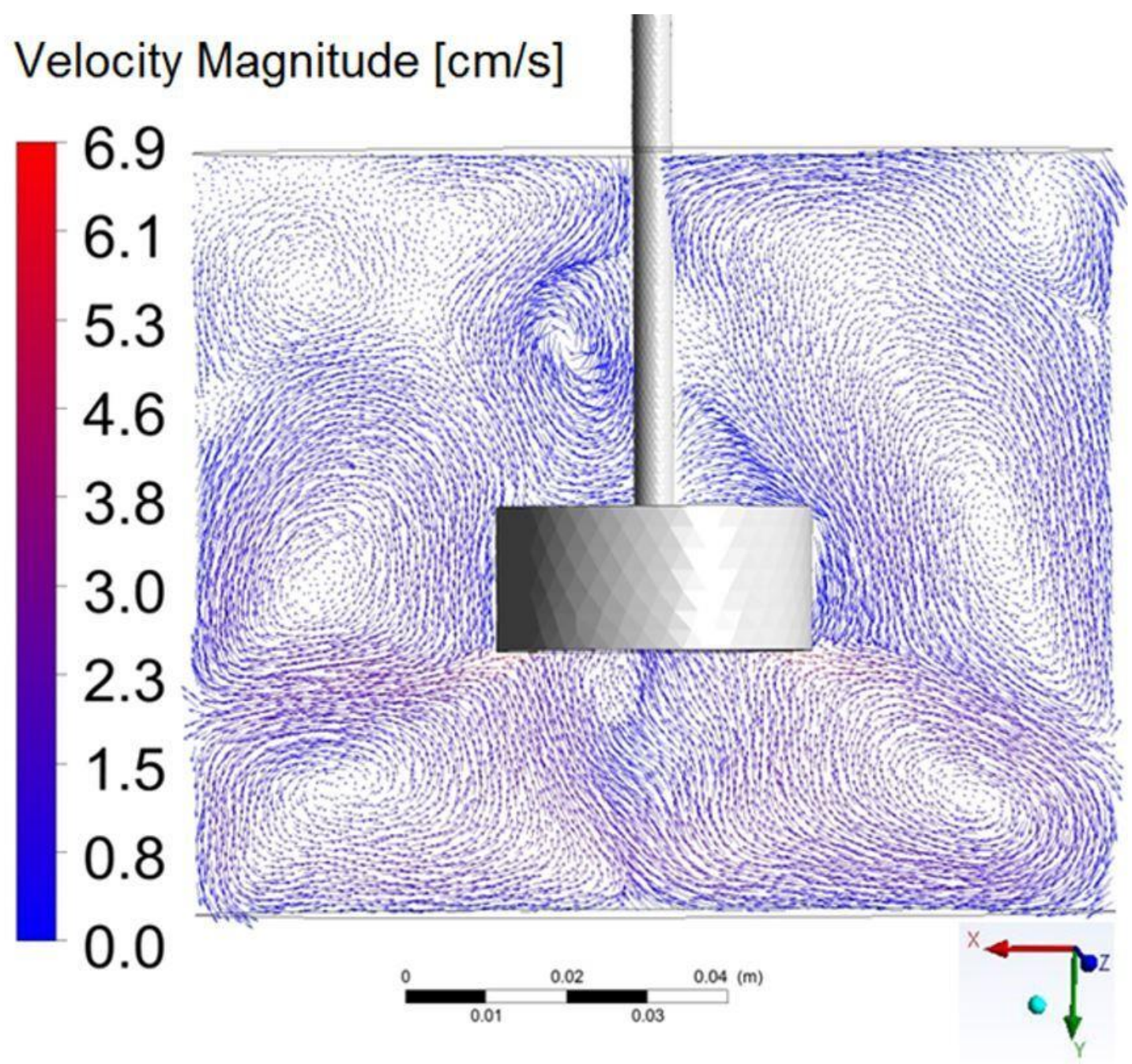

Fig. II-8 Velocity profile for reactor B with $1 \mathrm{cp} \mathrm{HCl}\left(15 \mathrm{wt} \%\right.$ at $86^{\circ} \mathrm{F}$ Nishikata et al. 1981) at $300 \mathrm{~s}$ with $100 \mathrm{rpm}$. Reactor height is $9.3 \mathrm{~cm}$ and reactor diameter is $11.4 \mathrm{~cm}$. Axial distance between the base of the disk and the bottom of the reactor is $3.2 \mathrm{~cm}$. Asymmetric flow is observed. Reprinted with permission from (Kotb and Nasr-El-Din 2020). 

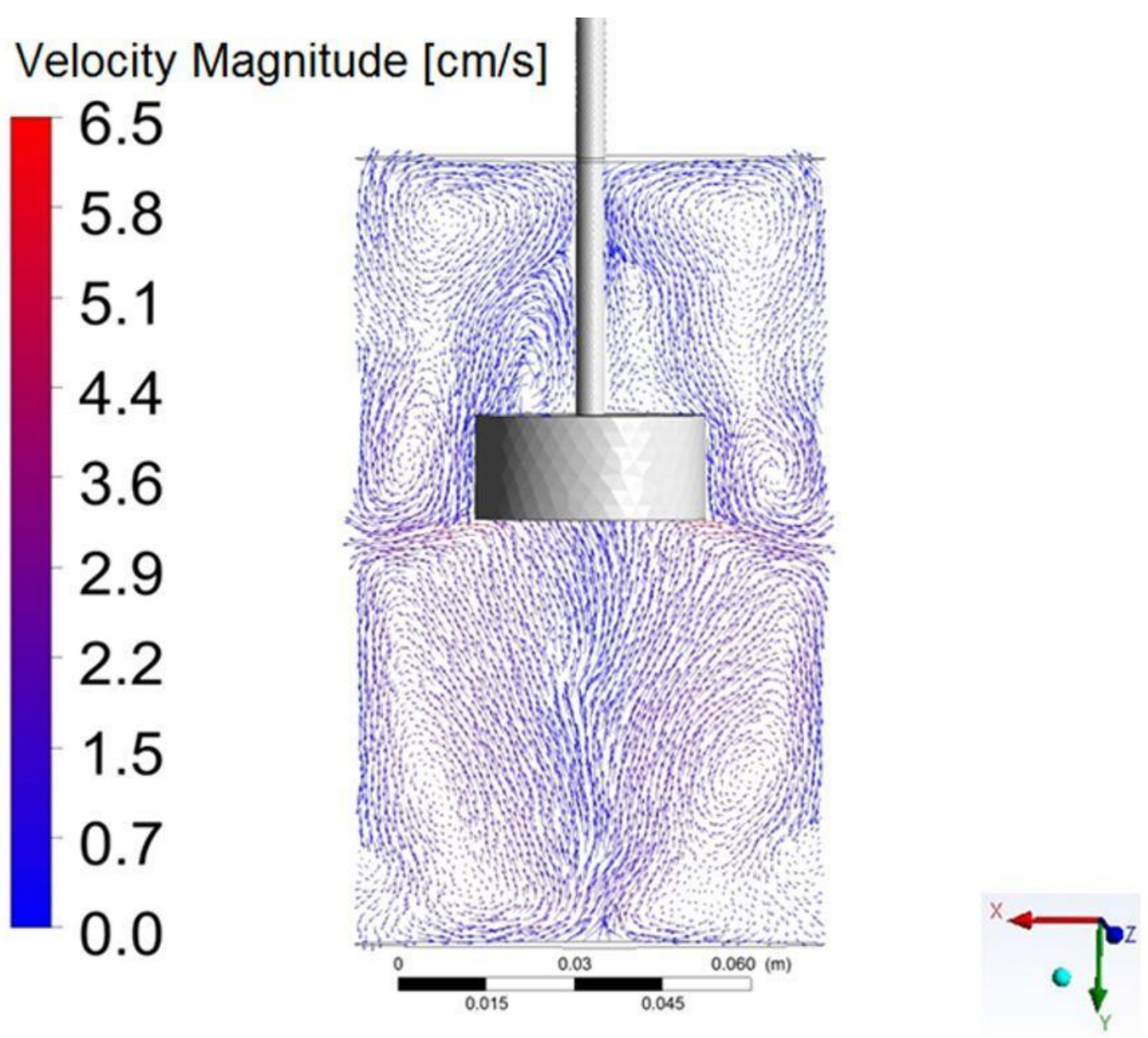

Fig. II-9 Velocity profile for reactor C with 1 cp $\mathrm{HCl}\left(15 \mathrm{wt} \%\right.$ at $86^{\circ} \mathrm{F}$ Nishikata et al. 1981) at $300 \mathrm{~s}$ with $100 \mathrm{rpm}$. Reactor height is $13.4 \mathrm{~cm}$ and reactor diameter is 8 cm. Axial distance between the base of the disk and the bottom of the reactor is 7.3 cm. Asymmetric flow is observed. Reprinted with permission from (Kotb and NasrEl-Din 2020). 
Rotational Speed (rpm)

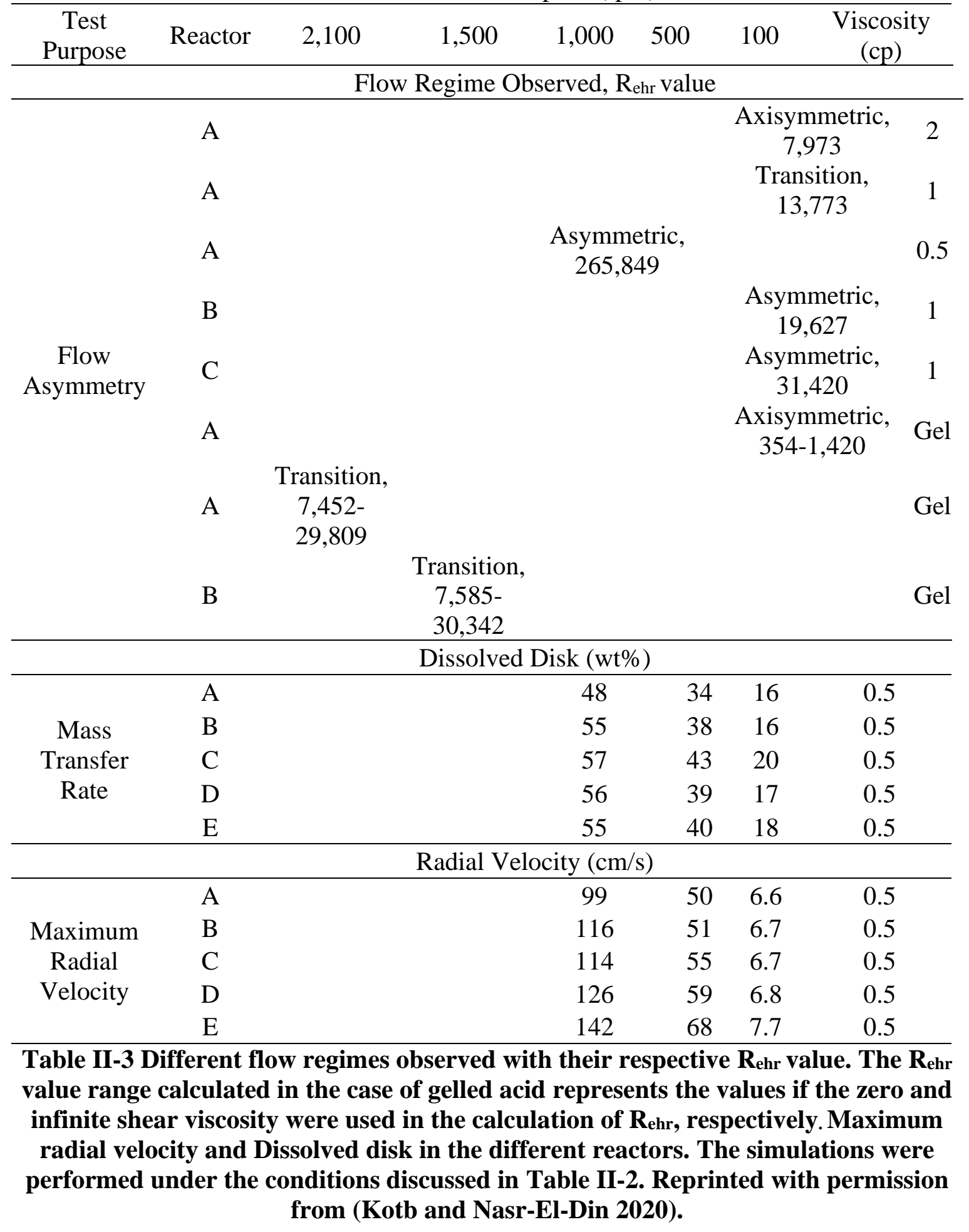


Impact of Reactor Dimensions on the Fluid Flow in Non-Newtonian Fluids.

Hansford and Litt's (1968) equation for non-Newtonian fluids assumes a constant shear and strain rate on the surface of the disk. Hansford and Litt (1968) also observed axisymmetric flow below the rotating disk. Gelled acid (1.5 wt $\%$ polymer concentration and $5 \mathrm{wt} \% \mathrm{HCl}$ concentration) was simulated to study asymmetry in non-Newtonian fluids. The properties of the gelled acid at $122^{\circ} \mathrm{F}$ were obtained from Nasr-El-Din et al. (2008). In order to model the viscosity of the non-Newtonian fluid, the Carreau model (Bird and Hassager 1987) was used with a zero-shear viscosity value of $40 \mathrm{cp}$, an infinite shear viscosity of $10 \mathrm{cp}$, a time constant of $0.01667 \mathrm{~s}$, and a power-law index of 0.587 . Fig. II-10 shows the relationship between viscosity and shear rate for the gelled acid (NasrEl-Din et al. 2008).

Fig. II-11 and Fig. II-12 show the velocity and strain profiles, respectively, in reactor A for $100 \mathrm{rpm}$. The velocity profile under the rotating disk in Fig. II-11 is exhibiting axisymmetric flow because $\mathrm{R}_{\mathrm{ehr}}$ is smaller than 8,000 if the finite-shear or zero-shear viscosity value is used in the $\mathrm{R}_{\mathrm{ehr}}$ calculation. Fig. II-12 shows an unequal distribution of strain of the fluid on the surface of the disk ranging between 27 to $0 \mathrm{~s}^{-1}$. This finding contradicts the assumption in Hansford and Litt's (1968) equation and can explain the anomalies in the mass transfer curves observed in their work at rotational speeds lower than $100 \mathrm{rpm}$. A transition from axisymmetric velocity profiles can be observed for $\mathrm{R}_{\mathrm{ehr}}$ larger than 8,000, as shown in Fig. II-13 and Fig. II-14. 


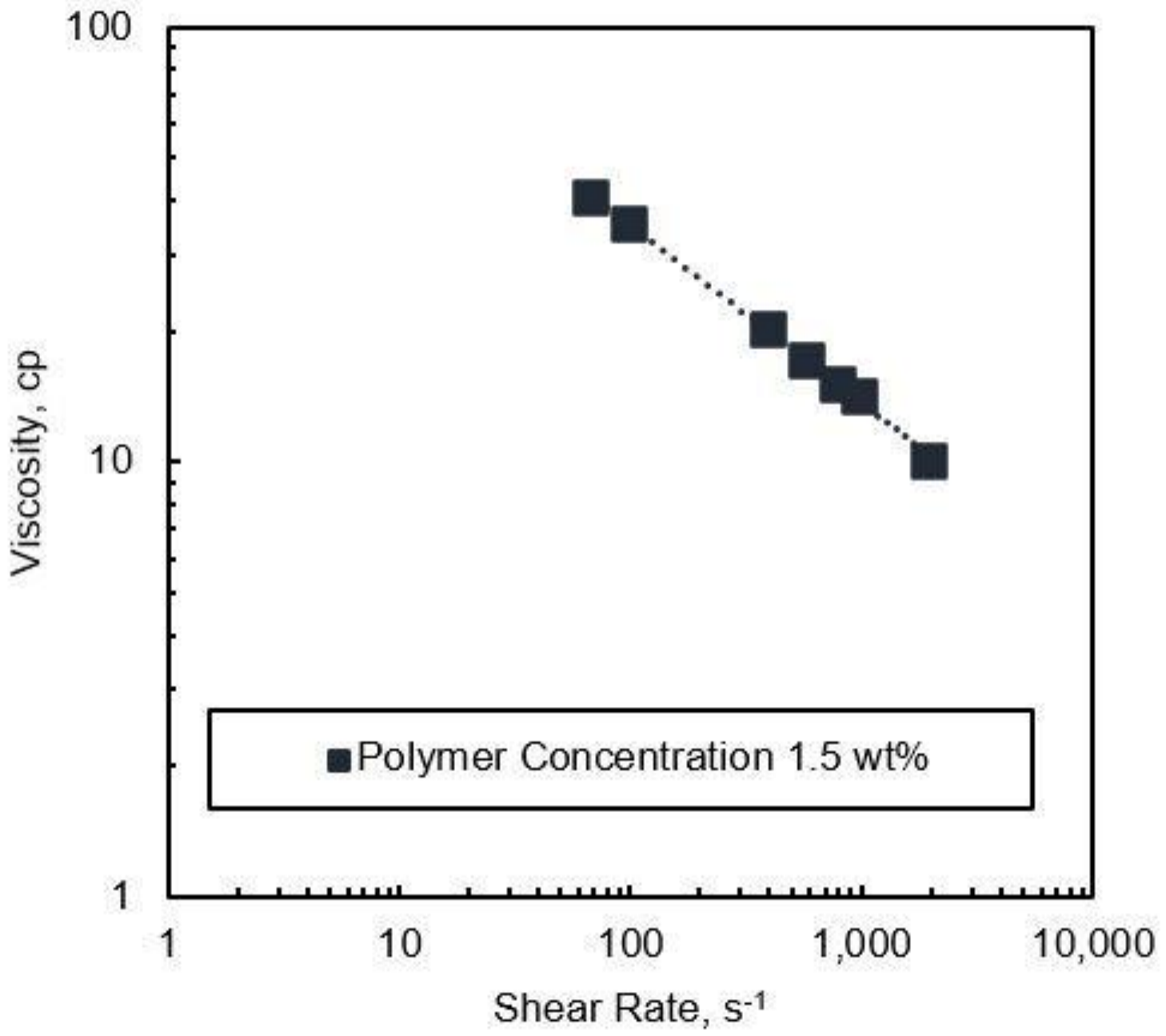

Fig. II-10 Effect of shear rate on the apparent viscosity of gelled acid (1.5 wt\% polymer concentration and $5 \mathrm{wt} \% \mathrm{HCl}$ ) at $1^{2}{ }^{\circ} \mathrm{F}$ (Nasr-El-Din et al. 2008). Reprinted with permission from (Kotb and Nasr-El-Din 2020). 


\section{Velocity Magnitude $[\mathrm{cm} / \mathrm{s}]$}

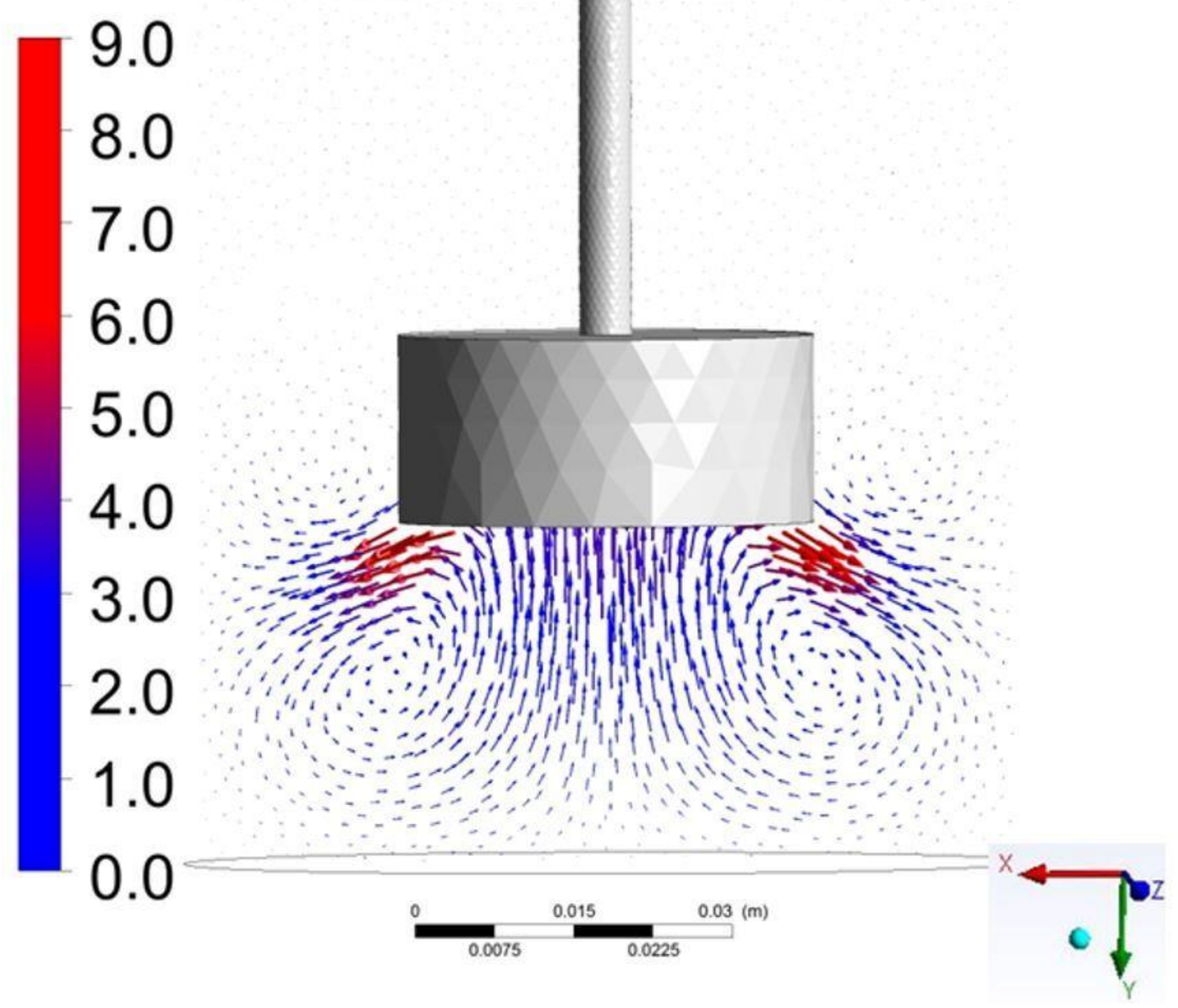

Fig. II-11 Velocity profile for reactor A with gelled acid (1.5 wt\% polymer concentration and $5 \mathrm{wt} \% \mathrm{HCl}$ at $1^{\circ} \mathrm{F}$ Nasr-El-Din et al. 2008) at $300 \mathrm{~s}$ with 100 rpm. Reactor height is $9.3 \mathrm{~cm}$ and reactor diameter is $8 \mathrm{~cm}$. Axial distance between the base of the disk and the bottom of the reactor is $3.2 \mathrm{~cm}$. Axisymmetric flow is observed. Reprinted with permission from (Kotb and Nasr-El-Din 2020). 

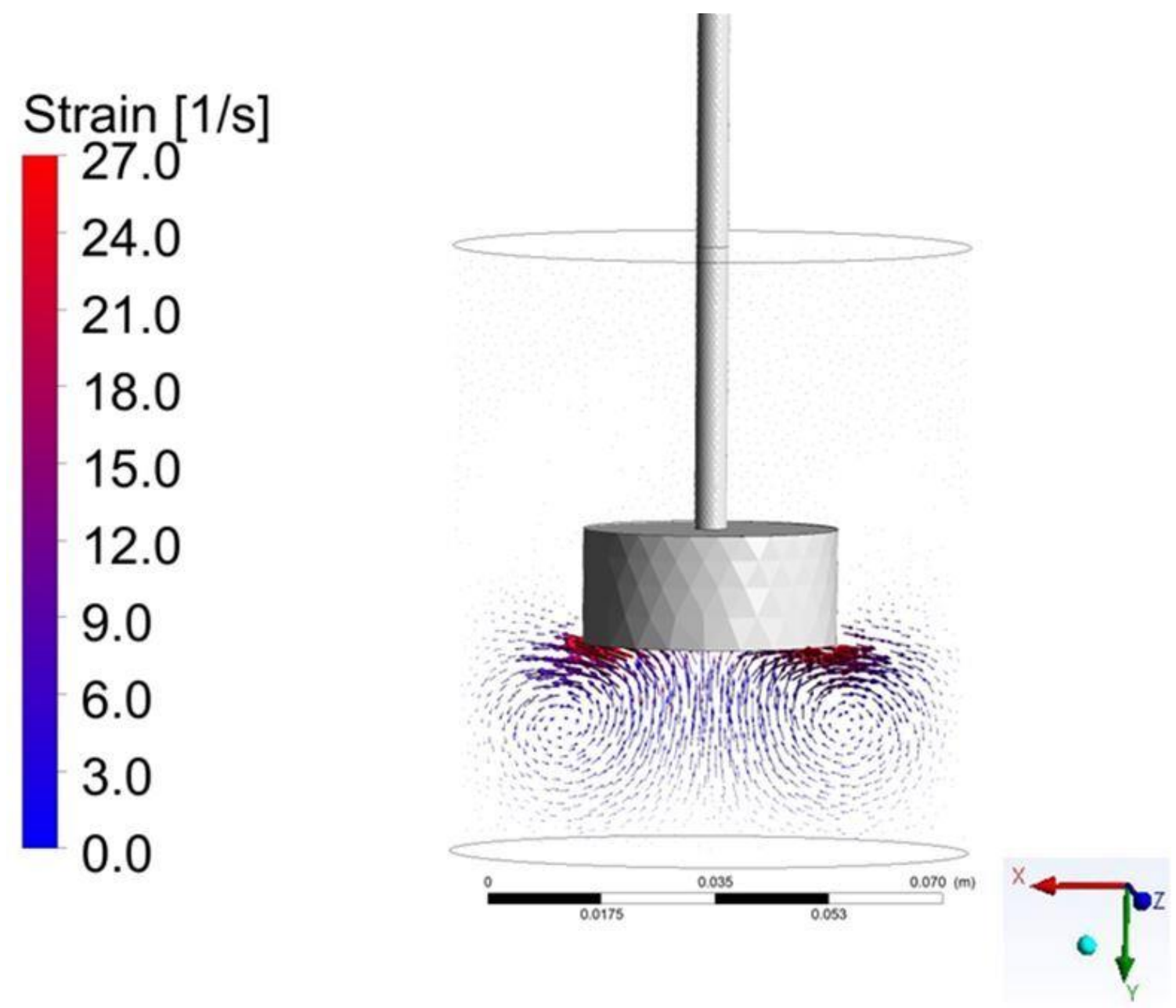

Fig. II-12 Strain profile for gelled acid $(1.5 \mathrm{wt} \%$ polymer concentration and $5 \mathrm{wt} \%$ $\mathrm{HCl}$ at $122^{\circ} \mathrm{F}$ Nasr-El-Din et al. 2008) in reactor A at $300 \mathrm{~s}$ with $100 \mathrm{rpm}$. Reactor height is $9.3 \mathrm{~cm}$ and reactor diameter is $8 \mathrm{~cm}$. Axial distance between the base of the disk and the bottom of the reactor is $3.2 \mathrm{~cm}$. Reprinted with permission from (Kotb and Nasr-El-Din 2020). 

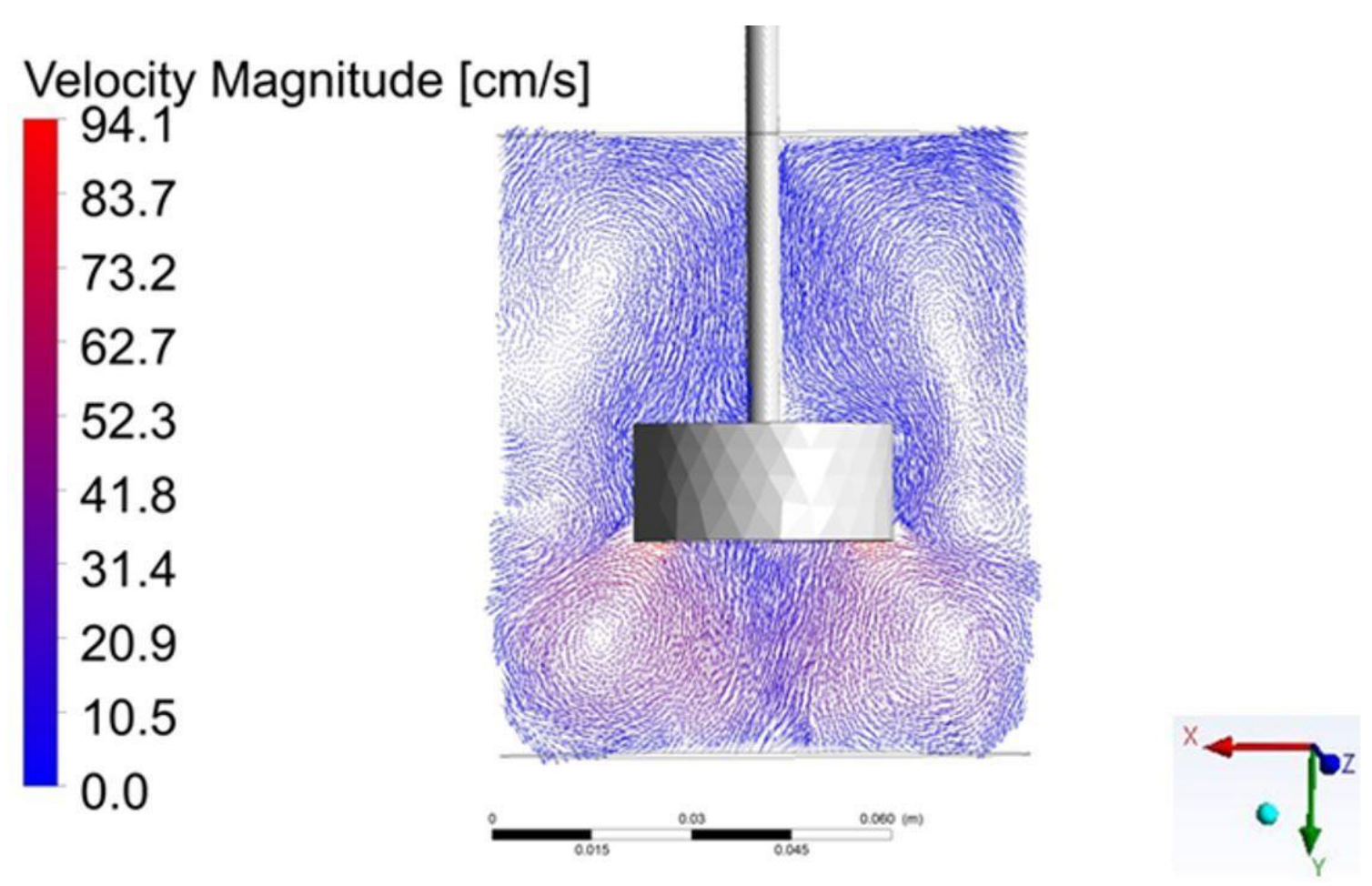

Fig. II-13 Velocity profile for reactor $A$ with gelled acid (1.5 wt\% polymer concentration and $5 \mathrm{wt} \% \mathrm{HCl}$ at $1^{\circ}{ }^{\circ} \mathrm{F}$ Nasr-El-Din et al. 2008) at $300 \mathrm{~s}$ with 2,100 rpm. Reactor height is $9.3 \mathrm{~cm}$ and reactor diameter is $8 \mathrm{~cm}$. Axial distance between the base of the disk and the bottom of the reactor is $3.2 \mathrm{~cm}$. Transition from axisymmetric flow is observed. Reprinted with permission from (Kotb and Nasr-ElDin 2020). 

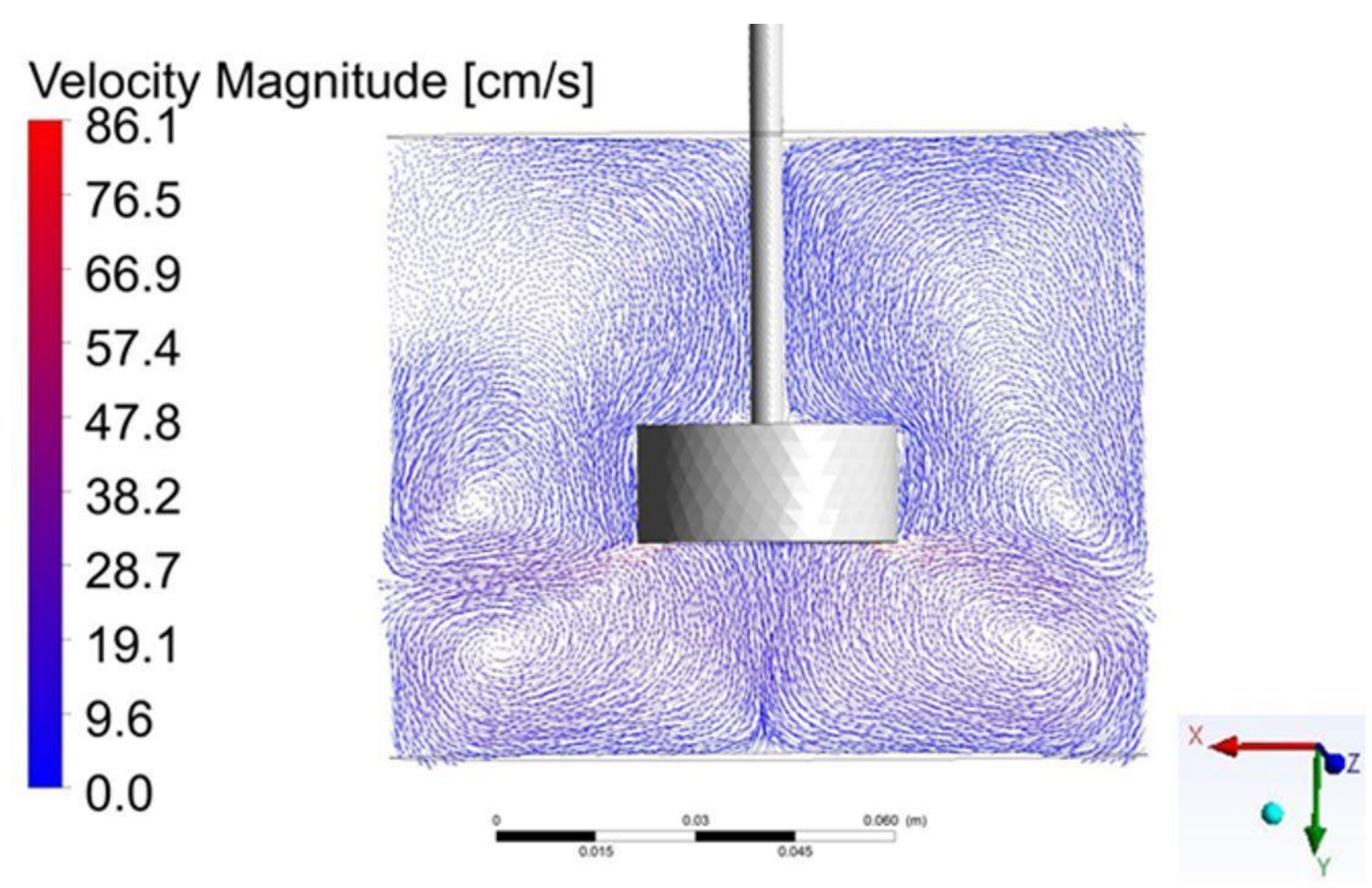

Fig. II-14 Velocity profile for case B with gelled acid (1.5 wt\% polymer concentration and $5 \mathrm{wt} \% \mathrm{HCl}$ at $1^{\circ 2} \mathrm{~F}$ Nasr-El-Din et al. 2008) at $300 \mathrm{~s}$ with 1,500 rpm. Reactor height is $9.3 \mathrm{~cm}$ and reactor diameter is $11.4 \mathrm{~cm}$. Axial distance between the base of the disk and the bottom of the reactor is $3.2 \mathrm{~cm}$. Transition from axisymmetric flow is observed. Reprinted with permission from (Kotb and Nasr-El-Din 2020).

Impact of Reactor Dimensions on the Mass Transfer of $\mathrm{H}^{+}$to the Disk in Newtonian

\section{Fluids}

Newman's (1966) equation assumes that the dimensions of the reactor compared to the disk are large enough for the fluid not to be impacted by the boundaries of the reactor. However, Lehmkuhl and Hudson (1971) reported that only the axial distance between the base of the disk and the bottom of the reactor impacts the $\mathrm{H}^{+}$transfer to the disk when tested with cinnamic acid and stainless-steel disk. In order to investigate these assumptions, the disks dissolved in the reactors Table II-1 were compared under the same 
conditions (Table II-2) at rotational speeds 1,000, 500, and $100 \mathrm{rpm}$. The comparison between the amount of disk dissolved in the five reactors is reported in Table II-3. At 100 $\mathrm{rpm}$, the results are almost identical for reactors A and B having the minimum rock dissolved at $16 \mathrm{wt} \%$. At 500 and 1,000 rpm, the disk in reactor A dissolved the least, with the other reactors having higher values. The results from the mass-transfer calculation support these findings (Fig. II-15) with the slope of the rate of disk dissolution ranging between $3.9 \mathrm{E}-6$ and $5.4 \mathrm{E}-6 \mathrm{gmol} / \mathrm{cm}^{2} \bullet$ s for reactors A and E, respectively. The relative difference in the rate of disk dissolution ranged between $28 \%$ between reactors $\mathrm{A}$ and $\mathrm{E}$ to $5 \%$ between reactors $\mathrm{D}$ and $\mathrm{E}$. As a result, the reactor that minimizes the impact of the boundaries on the mass transfer of $\mathrm{H}^{+}$to the disk is $\mathrm{D}$ because increasing the dimensions only increases the rate of mass transfer by $5 \%$, which can be attributed to computational error and can be neglected. In order to understand these findings, the radial velocities were observed because the maximum radial velocity of the fluid exposed to the disk will impact the rate of disk dissolution. Litt and Serad (1964) also reported that the dimensions of the reactor in a rotating disk application impact the radial and tangential velocity of the fluid. Table II-3 shows the maximum radial velocity of the fluid in the five reactors at the different rotational speeds. At $100 \mathrm{rpm}$, the relative difference in velocity reached a maximum of $10 \%$, while at $1,000 \mathrm{rpm}$ the relative difference in velocities reached a maximum of $30 \%$ between the different reactors. This can explain the different rates of mass transfer of $\mathrm{H}^{+}$to the disks in the different reactors. These results partially agree with Lehmkuhl and Hudson (1971), as they tested disk rotational speeds up to $200 \mathrm{rpm}$. The results show that at low rotational speeds and dissolution rates, only the axial distance 
between the base of the disk and the bottom of the reactor influences the mass transfer of $\mathrm{H}^{+}$to the disk. However, the current results also show that at larger rotational speeds and dissolution rates, the diameter of the reactor impacts the reaction. This can be explained by the increasing relative difference in radial velocity as the reactor size increases. Fig. II-15 also shows the impact of the flow regime on the rate of disk dissolution. In Reactor A, the simulation was performed at rotational speeds ranging between 10 and 1,000 rpm. $\mathrm{R}_{\text {ehr }}$ is lower than 8,000 only in the 10 -rpm case, indicating axisymmetric flow. However, there is no inflection observed in the slope of the rate of disk dissolution between the 10rpm case and the remaining cases.

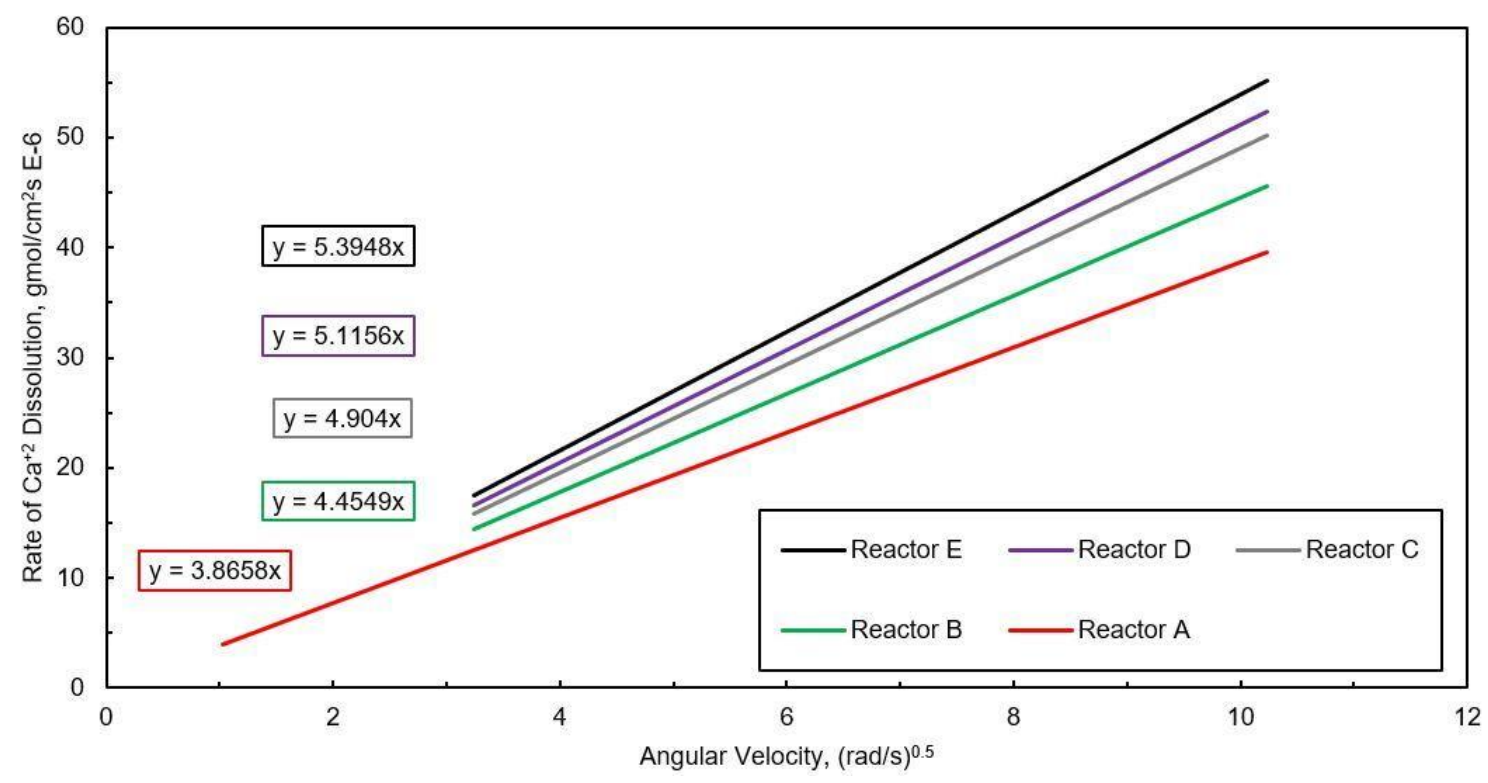

Fig. II-15 Rate of dissolution of the disk inside $15 \mathrm{wt} \% \mathrm{HCl}$ in the five reactors. Parameters discussed in Table II-2. Reprinted with permission from (Kotb and Nasr-El-Din 2020).

\section{Model Validation}


Fig. II-16 shows the three marble disks after the RDA experiments were performed at $150^{\circ} \mathrm{F}$ using $15 \mathrm{wt} \% \mathrm{HCl}$. The grooves on the disks, especially at higher disk rotational speeds, further confirm the unequal distribution of shear of the acid on the surface of the disk and can explain the shape of the disks. Results from the ICP measurement for the experiments performed are reported in Fig. II-17. The rate of $\mathrm{Ca}^{+2}$ dissolution was then calculated and plotted against the results obtained from the simulation performed at the same conditions (Fig. II-18). The experimental results match the simulation results performed in reactor $\mathrm{C}$. This difference between reactor A simulation and the experiment can be a result of the diffusion coefficient calculation that ignores the impact of the boundaries of the mass transfer of $\mathrm{H}^{+}$to the disk. Simulation tests were then performed on the testing conditions in Arslan et al. (2017). Table II-2 summarizes the conditions under which the simulations were performed. Fig. II-19 shows the comparison between the results obtained by Arslan et al. (2017) and the simulation results performed in the same conditions as discussed in Table II-2. The results reported by Arslan et al. (2017) (performed in reactor A dimensions) matched the simulated results in reactor D. The relative difference in the rate of disk dissolution ranged between $10 \%$ between reactors $\mathrm{A}$ and $\mathrm{E}$ to $2 \%$ between reactors $\mathrm{D}$ and $\mathrm{E}$. This decrease in relative difference between the five reactors for the conditions discussed in Arslan et al. (2017) from the conditions discussed in Table II-2 under mass transfer rate can be attributed to the overall decrease in the reactivity of the acid at the lower temperature. As the reactivity decrease, the change in the area of the disk throughout the experiment decreases. 


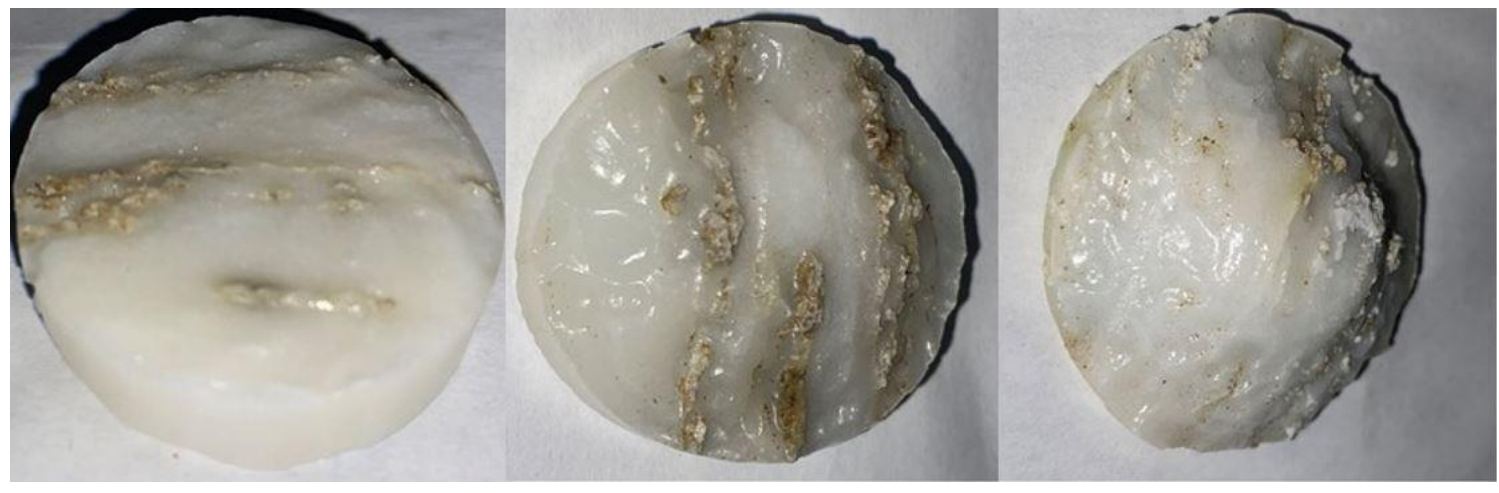

Fig. II-16 Marble disks from RDA experiments performed at $150^{\circ} \mathrm{F}$ using $15 \mathrm{wt} \%$ $\mathrm{HCl}$ and $1 \mathrm{wt} \%$ corrosion inhibitor. The disks rotational speeds from left to right were 132, 584, and 1,172 rpm. Reprinted with permission from (Kotb and Nasr-El-

Din 2020).

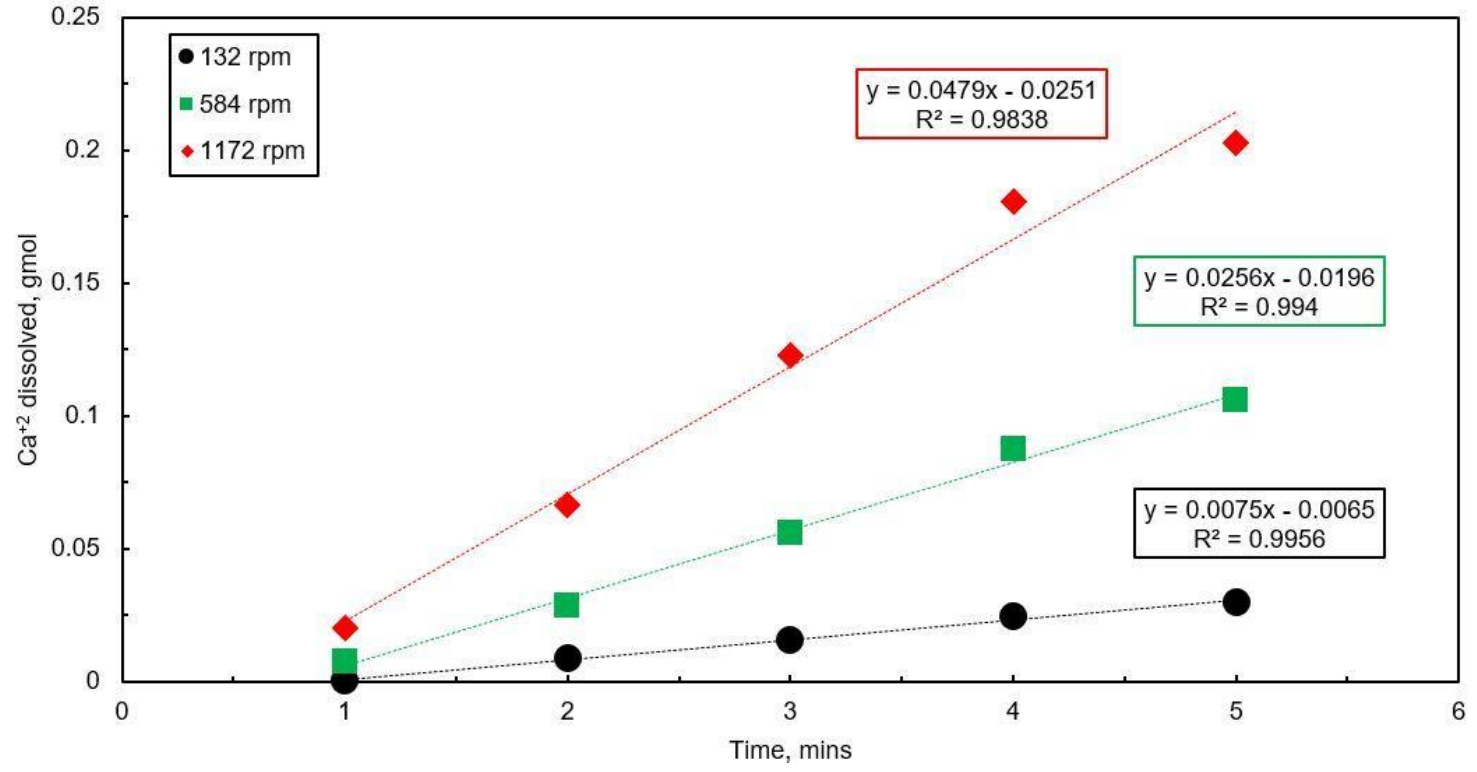

Fig. II-17 $\mathrm{Ca}^{+2}$ dissolved for the RDA experiment performed at $150^{\circ} \mathrm{F}$ using $15 \mathrm{wt} \%$ $\mathrm{HCl}$ and $1 \mathrm{wt} \%$ corrosion inhibitor. Results obtained from ICP measurements. Reprinted with permission from (Kotb and Nasr-El-Din 2020). 


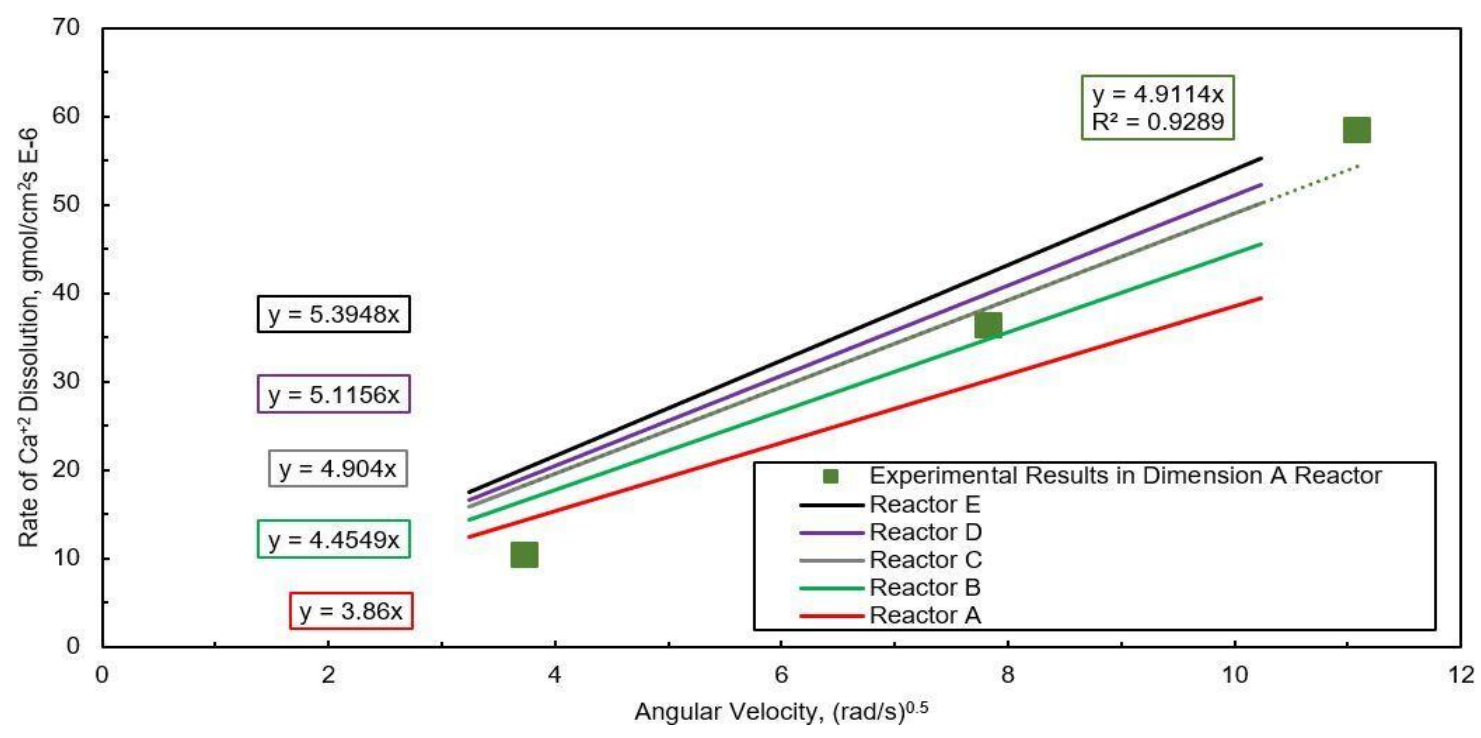

Fig. II-18 Comparison between the rate of dissolution of the disk inside $15 \mathrm{wt} \%$ $\mathrm{HCl}$ in the five reactors (parameters discussed in Table II-2) and the experimental results performed at the same conditions with reactor A dimensions. Reprinted with permission from (Kotb and Nasr-El-Din 2020).

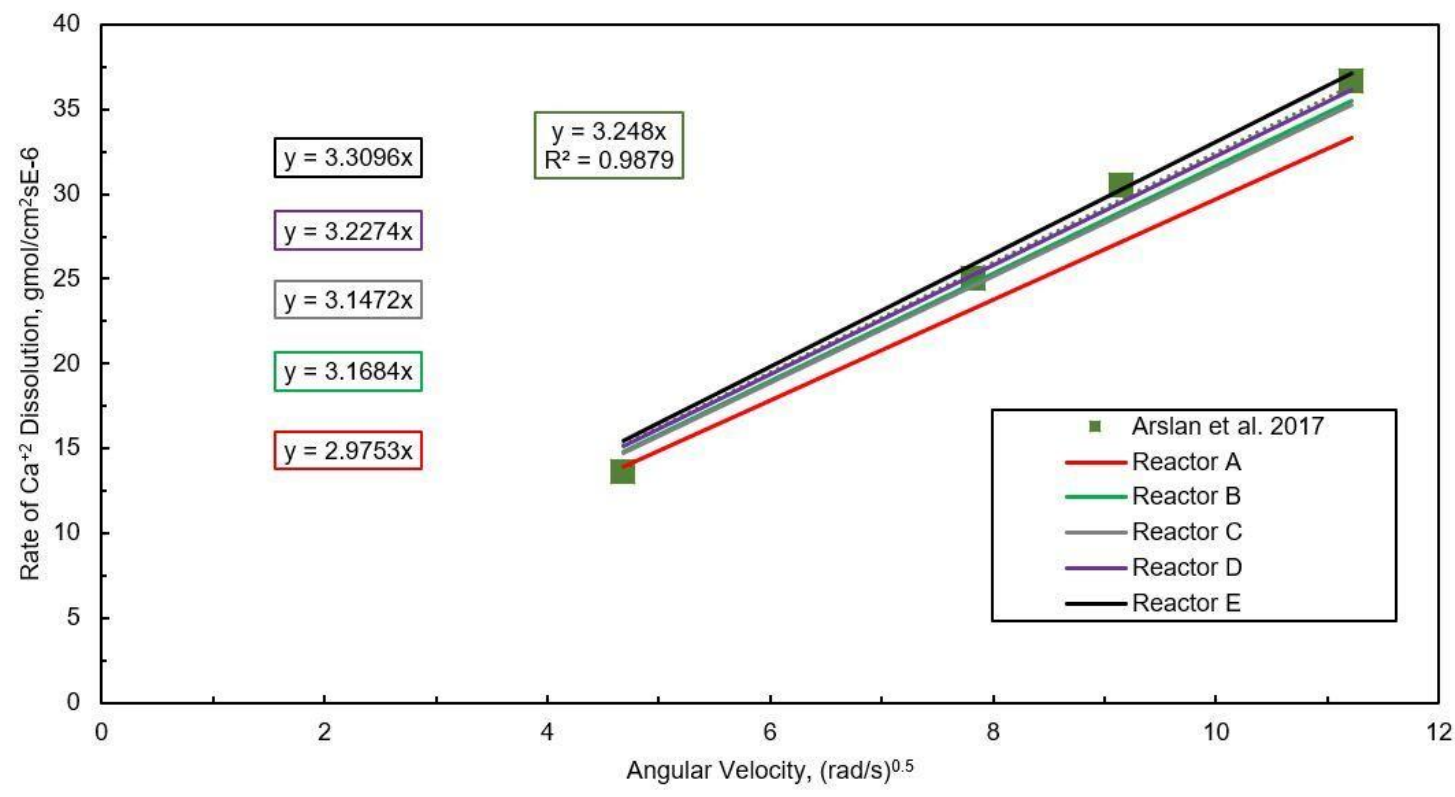

Fig. II-19 Comparison between the rate of dissolution of the disk inside $18.76 \mathrm{wt} \%$ $\mathrm{HCl}$ in the five reactors (parameters discussed in Table II-2) and the experimental results obtained by Arslan et al. (2017) with reactor A dimensions. Reprinted with permission from (Kotb and Nasr-El-Din 2020). 
CHAPTER III

TURBULENCE LEADS TO OVERESTIMATION OF THE ACID-DIFFUSION

COEFFICIENT AT TYPICAL EXPERIMENTAL CONDITIONS USING THE

ROTATING DISK APPARATUS*

Materials and Methods

Experimental Studies

Disks were cut from a slab of calcite marble, the essentially white solid rock with 8-10 in. spaced subparallel, about 0.2 in. thick gray streaks. Special care was taken to sample from the white areas composed of $>99.0 \mathrm{wt} \% \mathrm{CaCO}_{3}$, as confirmed by: (1) X-ray diffraction mineral composition analysis using Bruker D8 ADVANCE Eco diffractometer, and (2) microscopic examination using a TESCAN VEGA3 scanning electron microscope equipped with an energy-dispersive X-ray spectroscopy detector (SEM/EDS). To identify and quantify the mineral phases, all the recorded X-ray diffractograms were analyzed using Bruker EVA and TOPAS software programs, respectively. SEM/EDS analysis revealed that the samples of calcite marble were composed of grains with a maximum size of approximately 1000 and $150 \mu \mathrm{m}$.

Deionized (DI) water with a resistivity of $18.2 \mathrm{M} \Omega-\mathrm{cm}$ at room temperature was used in this study. Solutions of $1 \mathrm{~N}$ and $15 \mathrm{wt} \% \mathrm{HCl}$ were prepared from concentrated hydrochloric

\footnotetext{
* Part of this chapter is reprinted with permission from "Turbulence Leads to Overestimation of the AcidDiffusion Coefficient at Typical Experimental Conditions using the Rotating Disk Apparatus" by Ivanshin, I., Kotb, A., and Nasr-El-Din 2021, Journal of Petroleum Science and Engineering 205. Copyright 2021 by Elsevier.
} 
acid (ACS reagent grade) and DI water. 1 vol\% of corrosion inhibitor was added to the 15 wt $\% \mathrm{HCl}$ solution. The acid concentration was measured using a Metrohm 907 Titrando auto-titrator with $0.1 \mathrm{M}$ sodium hydroxide solution as a titrant. The kinematic viscosity of the acid solution was measured using a capillary viscometer.

Disks of around 0.5 in. thickness and three diameters $-0.72,1.11$, and 1.46 in. - were cut and prepared according to the sample preparation procedure of Fredd and Fogler (1998). Disks were successively polished using $120,200,400$ mesh sandpaper, then soaked in $0.1 \mathrm{~N} \mathrm{HCl}$ for 30 minutes, and thoroughly rinsed in DI water.

The dissolution experiments were performed using an RDA CRS-100 manufactured by CoreLab Instruments ltd. A description of this equipment and its operation appears in Taylor et al. (2004). A schematic of the RDA used in this work is shown in Fig. III-1. Disks were attached to the rotating shaft using a heat-shrink tube such that only the bottom face of the disk was exposed to the acid solutions at the following conditions:

- $15 \mathrm{wt} \% \mathrm{HCl}$ at $100^{\circ} \mathrm{F}$ and disk rotational speeds of $207,380,587,829,1,175$, and $1,555 \mathrm{rpm}$.

- $\mathrm{HCl}$ at $73.4^{\circ} \mathrm{F}$ (ambient temperature) and disk rotational speeds of 518 and 1,175 rpm.

Experiments were performed for 5 minutes while maintaining a nitrogen pressure of $1,100-1,150$ psig in the reaction vessel to keep $\mathrm{CO}_{2}$ in solution and maintain a singlephase fluid (Welton and Domelen 2008; Nasr-El-Din et al. 2008; Taylor and Nasr-El-Din 2009). Samples of acid $\left(3 \mathrm{~cm}^{3}\right)$ were withdrawn from the reaction vessel every minute, and the sampling line was purged with air after each sample was withdrawn to prevent 
sample contamination. After the final acid sample was withdrawn at the five-minute mark of the RDA experiment, it took 80-90 seconds to depressurize the reservoir and reactor vessels, drain the acid solution left in the reactor vessel and remove the reacted disk. Calcium ion concentrations in the withdrawn acid samples were determined using a PerkinElmer Optima 7000 DV Inductively Coupled Plasma-Optical Emission Spectrometer (ICP-OES, hereafter ICP). The mass-transfer $\left(\mathrm{H}^{+}\right)$rates and acid-diffusion coefficients were calculated based on the calcium ion concentrations in the first four withdrawn acid samples using Newman's equation (1966). Correlation coefficients of $\geq$ 0.994 were obtained for all experiments to provide accurate mass-transfer rate data. Certain experiments were performed in duplicate to assess the reproducibility of the results. 


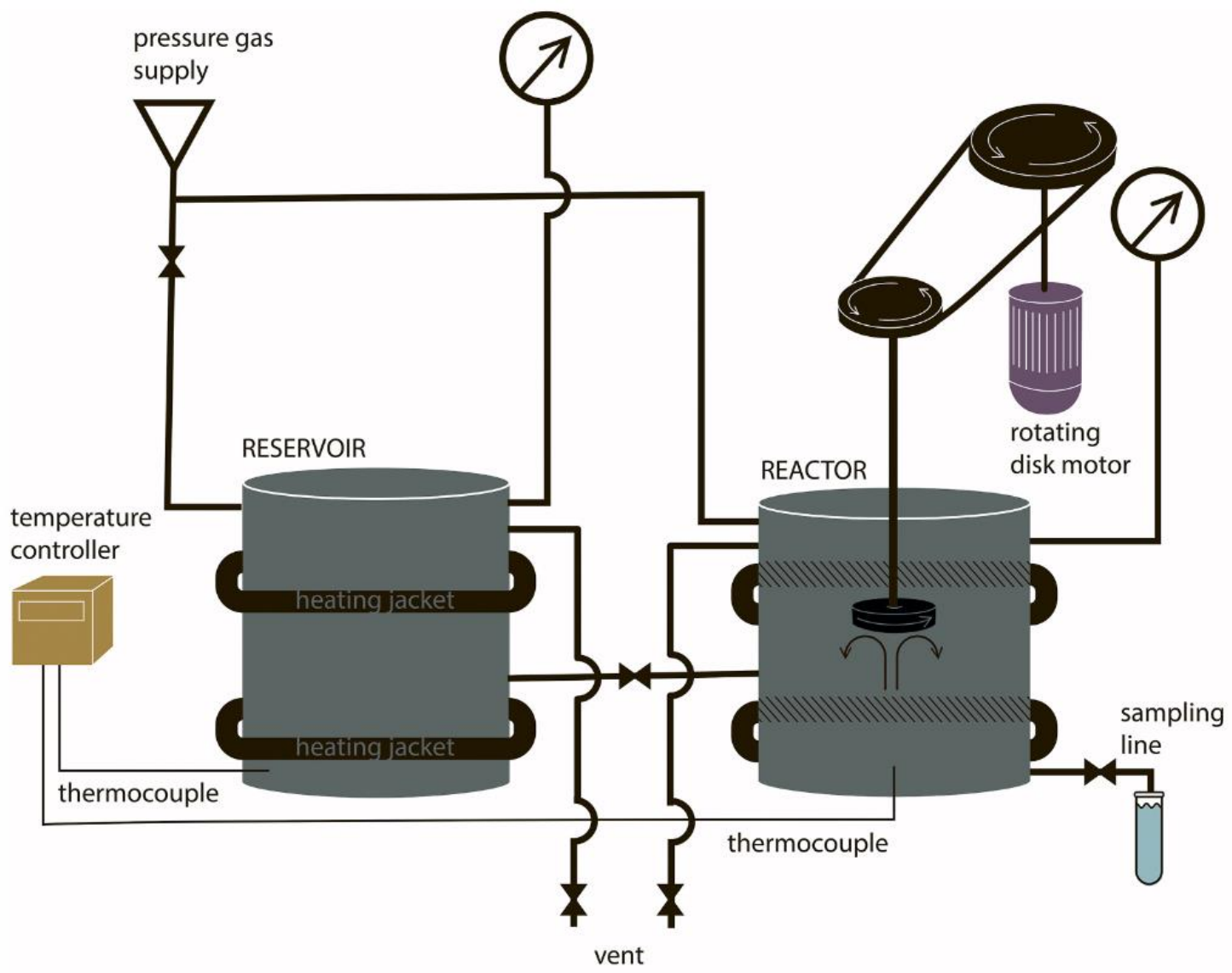

Fig. III-1 A schematic diagram for the rotating disk. Reprinted with permission from (Ivanshin et al. 2021).

\section{Simulation Studies}

Disks of three diameters were simulated (Table III-1) to study the effect of disk dimensions on the velocity profile and the resulting mass transfer of $\mathrm{H}^{+}$at the disk surface. Reactors simulated dimension match the reactor used in the experiment with $8 \mathrm{~cm}$ diameter, $9.5 \mathrm{~cm}$ height, and $5 \mathrm{~cm}$ axial distance between the bottom of the disk and the bottom of the reactor. 


\begin{tabular}{cc} 
Disk Diameter (in.) & $\begin{array}{c}\text { Number of Cells } \\
\text { (Million) }\end{array}$ \\
\hline 1.5 & 1.2 \\
1 & 4 \\
0.75 & 4.5 \\
\hline
\end{tabular}

\section{Table III-1 Number of cells used for the different disk sizes. Reprinted with permission from (Ivanshin et al. 2021).}

The steps followed to develop the computational fluid-dynamics model in this work are discussed by Zhang et al. (2014), Ali et al. (2019), Kotb and Nasr-El-Din (2020), and Kotb et al. (2021). The two-scale (average-continuum) approach was applied to simulate the disk dissolution during the RDA experiment. ANSYS ${ }^{\circledR}$ Fluent modeling software (ANSYS, Inc, Canonsburg, Pennsylvania, USA) was used to solve the mass-continuity, momentum, and transport equations using the finite-volume method. The simulation was performed at experimental conditions discussed previously. Since the diffusion coefficient and reaction-rate coefficient are needed to run the simulation, but are not available before running the experiment, the values were obtained from the literature (Peng et al. 2015; Kotb and Nasr-El-Din 2020) as $3.13 \times 10^{-5} \mathrm{~cm}^{2} / \mathrm{s}$ and $7.7 \mathrm{~cm} / \mathrm{s}$, respectively. In order to determine the optimum number of cells for the meshes at different disk sizes, the simulations were run at different mesh sizes for each disk size. Once the relative change in the amount of rock dissolved between two successive mesh sizes dropped to less than $5 \%$, that mesh was selected. A relative difference that small can be attributed to computational error and can be neglected. Information about the reactor sizing, grid independence test, and model development is discussed in Kotb and Nasr-El-Din (2020). Boundary conditions in the model were set to the interior of the disk and acid with a no- 
slip boundary condition on the disk surface and were set to the wall for the reactor boundaries and disk holder boundaries. The pressure velocity coupling used was the semiimplicit method for the pressure-linked equations, which is recommended for steady-state cases with some complexities as present in this case (Jang et al. 1986). The software then checks for convergence or performs additional iterations before moving to the next timestep. Convergence is achieved when the residual from continuity and velocity drops below 0.001 . This procedure continues until the end of the test duration. The simulations are run on a high-performance computing facility, which provides parallel processing capabilities that divide the model into different parts that are solved simultaneously. The computational time can take up to 3 days.

Table III-1 summarizes the number of cells used for simulating the reaction at each disk size. The number of elements in the mesh should be decreasing with decreasing disk size. However, for the 1 and 0.75 in. disks, the smaller element number resulted in a calculation error that stopped the simulation from converging. As a result, larger element numbers were chosen to prevent divergence of the simulation. The simulation results were then used to obtain the dissolution rate and visualize the velocity vectors under the disk. The rate of mass transfer of $\mathrm{Ca}^{+2}$ was then calculated using the following equation:

$$
J_{m t}=\frac{C_{r} \alpha^{\prime} \omega^{0.5}}{t A_{d}},
$$


where $C_{r}$ is the total calcium ion concentration in the disk, $\alpha$ is the amount of rock dissolved, $t$ is the test duration, and $A_{d}$ is the initial area of the disk exposed to the acid calculated using:

$A_{d}=\pi\left(r^{\prime}\right)^{2}$

where $r^{\prime}$ is the radius of the disk. The rate of mass transfer of $\mathrm{Ca}^{+2}$ was then multiplied by two to get the mass transfer of $\mathrm{H}^{+}$to the disk since two molecules of $\mathrm{HCl}$ are needed to react with one molecule of $\mathrm{CaCO}_{3}$. The results were then used to study the impact of the rock diameter on the mass transfer of $\mathrm{H}^{+}$to the disk.

\section{Results and Discussion}

\section{Experimental Dissolution in $15 \mathrm{wt} \% \mathrm{HCl}$ at $100^{\circ} \mathrm{F}$}

For flow at the surface of the $1.46 \mathrm{in}$. disks rotating at $1,555 \mathrm{rpm}$, the $\operatorname{Re}=4.9 \times 10^{4}$. This value is almost one order of magnitude lower than the commonly assumed $\operatorname{Re}_{\mathrm{cr}}=3 \times 10^{5}$, indicating that flow in all the RDA experiments should be in the laminar regime. According to Newman's equation (1966), the experimental data plotted as the masstransfer rate over the square root of the disk rotational speed should fall on the straight line passing through the origin. Fig. III-2 shows these graphs for the disks of three diameters. The data points are well fitted with straight lines, but their slope increases with the disk diameter. Because of that, the calculated values of the diffusion coefficient are $6.71 \times 10^{-5}, 5.01 \times 10^{-5}$, and $3.08 \times 10^{-5} \mathrm{~cm}^{2} / \mathrm{s}$ for $1.46,1.11$, and $0.72 \mathrm{in}$. disks, respectively. These results contradict the theoretical assumption of uniform accessibility of the disk 
surface at $\operatorname{Re}<\operatorname{Re}_{c r}$. Because the rate of mass transfer is calculated per unit area, its value should not depend on the disk diameter in the entire range of the rotational speeds tested.

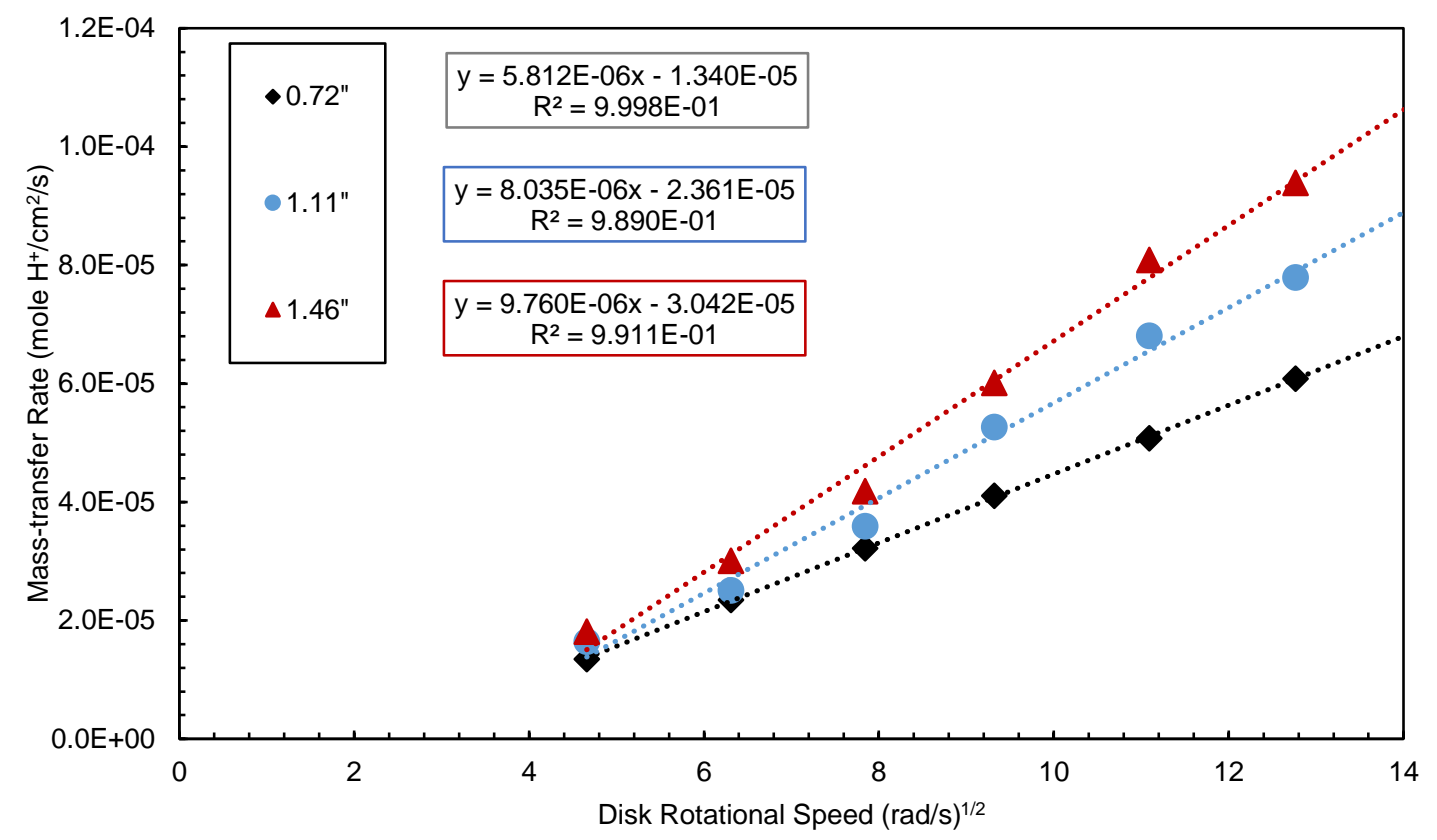

Fig. III-2 Mass-transfer rate to the disks of calcite marble as a function of the disk rotational speed in $15 \mathrm{wt} \% \mathrm{HCl}$ at $100^{\circ} \mathrm{F}$. Reprinted with permission from (Ivanshin et al. 2021).

The appearance of the disks after the dissolution tests (Fig. III-3 through Fig. III-6) can explain these inconsistencies. At low disk rotational speeds of 207 and $380 \mathrm{rpm}$, the surfaces of the disks of all diameters are fairly smooth with shallow curved etch lines that originate close to the center and propagate to the edge of the disk. This pattern is in accordance with the theoretical and experimental distribution of streamlines (Fig. III-4a and Fig. III-4b) in the laminar flow regime presented by Levich (1962). As the disk rotational speed increases, cavities appear initially close to the edge of the disks (Fig. 
III-5a), and then occupy a larger area on the surface propagating toward the center (Fig. III-5b). At the same time, cavities decrease in size, and a hump of less-dissolved rock becomes evident at the centers of the disks, where the laminar flow regime is sustained. This phenomenon starts at a lower rotational speed and is more evident on the disks that have larger diameter. Cavities appear close to the edge of 1.46 and 1.11 in. disks at rotational speeds of 587 and $829 \mathrm{rpm}$, or Reynolds numbers of $1.9 \times 10^{4}$ and $1.5 \times 10^{4}$, respectively. Contrary to the established theory, starting from these experimental conditions, the surface of the disk becomes non-uniformly accessible.

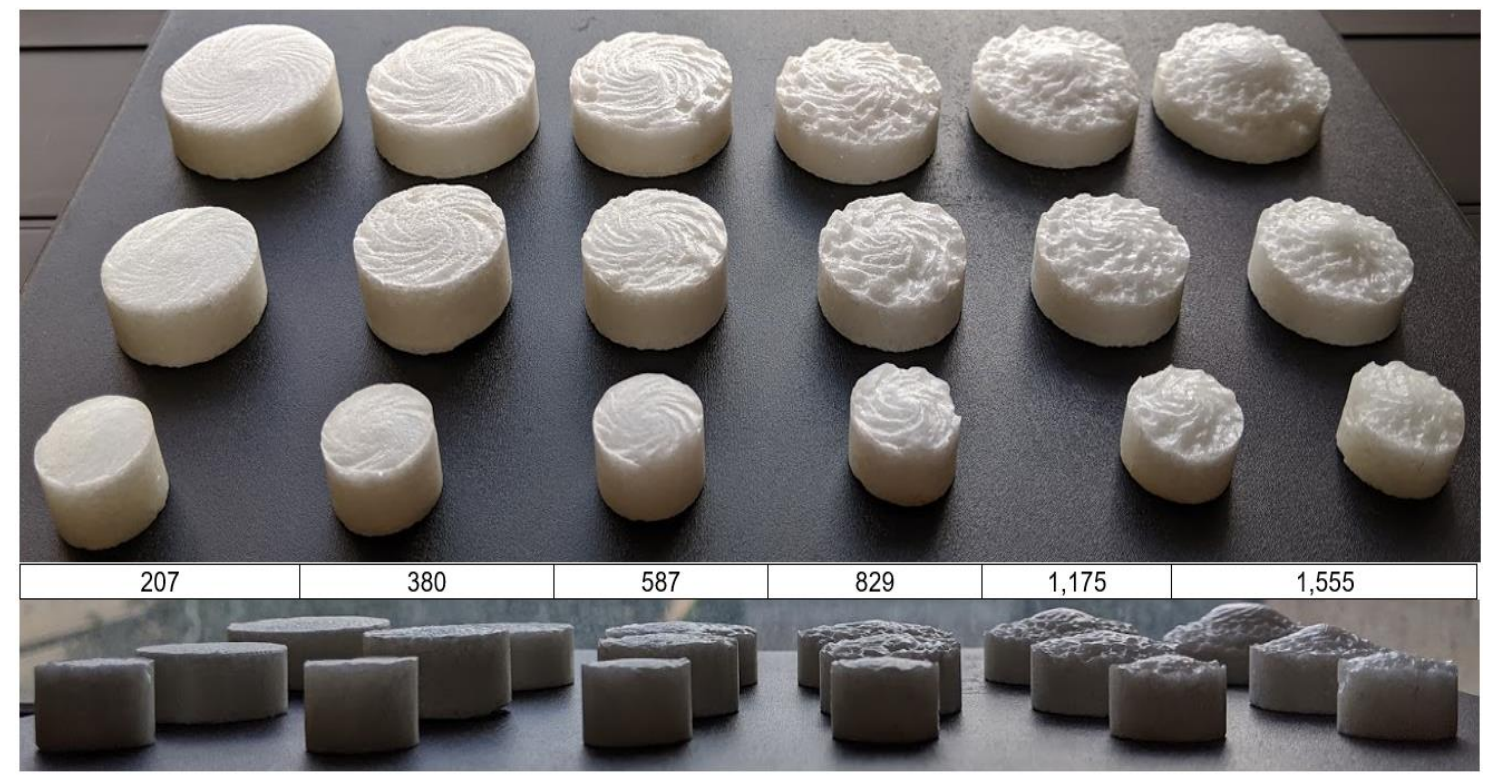

Fig. III-3 Disks of calcite marble after the dissolution experiments. Moving forward from back - disks of 1.46, 1.11, and 0.72 in. diameter. Moving from left to right disk rotational speed of $207,380,587,829,1,175$, and $1,555 \mathrm{rpm}$. Reprinted with permission from (Ivanshin et al. 2021). 


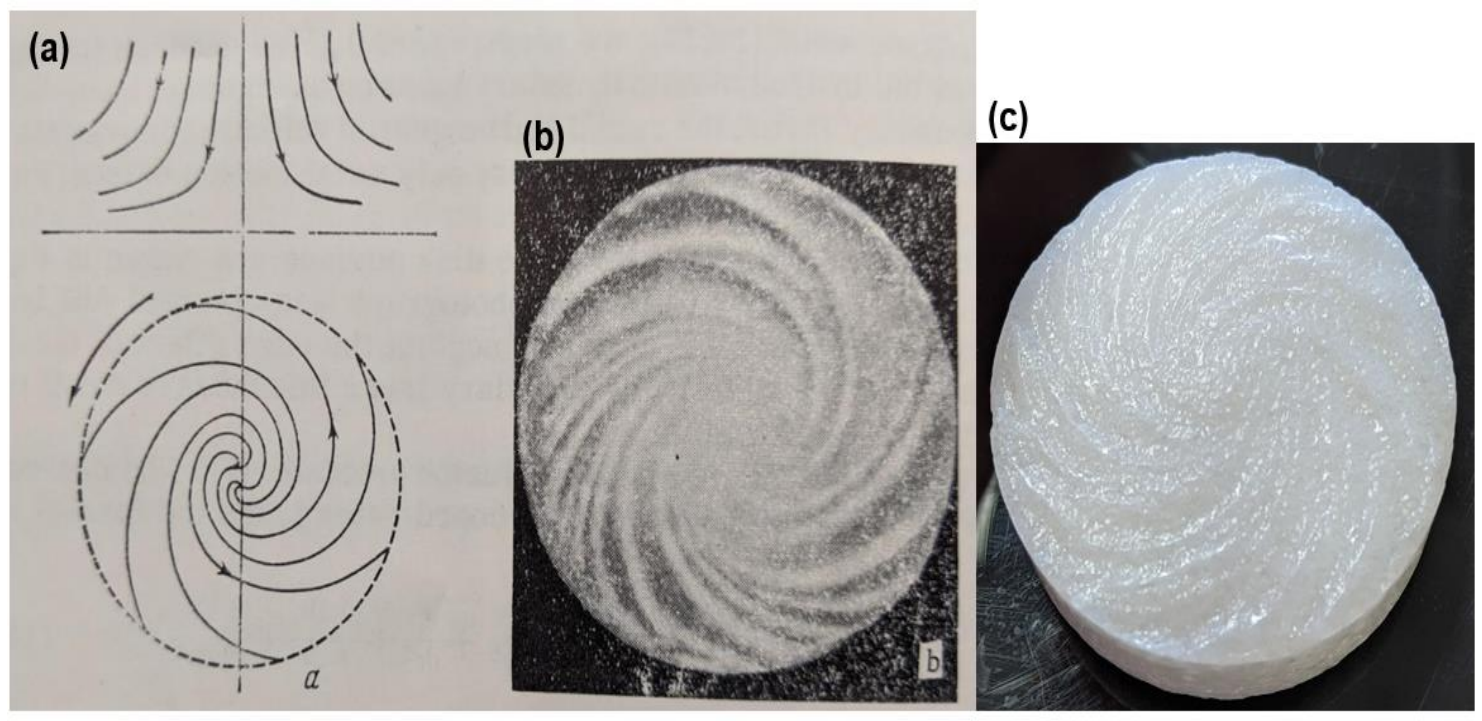

Fig. III-4 Distribution of streamlines at the surface of the RD: (a) schematic; (b) etched lines on the RD (after Levich 1962); (c) 1.46 in. disk after RDA test at 380 rpm (present study). Reprinted with permission from (Ivanshin et al. 2021).

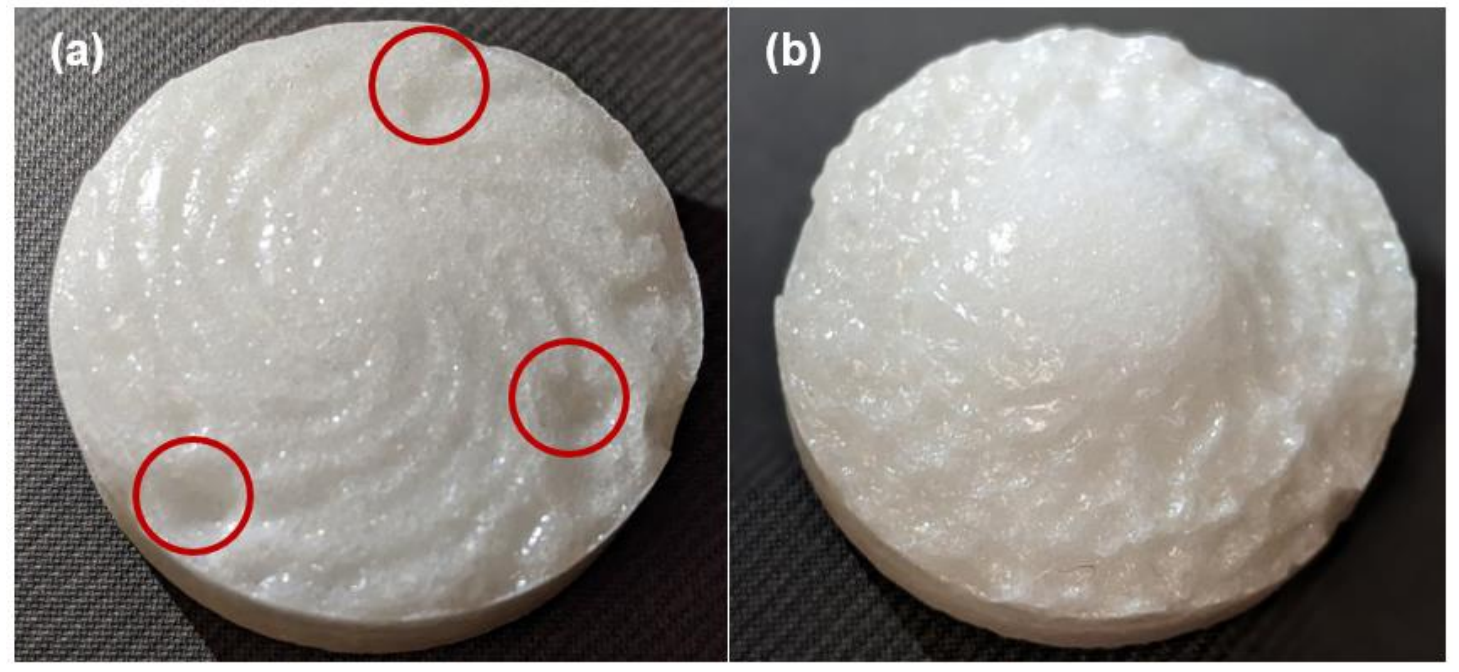

Fig. III-5 Progressive loss of uniform accessibility of the surface of 1.46 in. disk during RDA tests at $587 \mathrm{rpm}$ (a) and 1,555 rpm (b). Note several comparably large cavities at the outer region of the disk in (a), and smaller size cavities covering the most of the disk surface with smooth hump at the center of the disk in (b).

Reprinted with permission from (Ivanshin et al. 2021). 


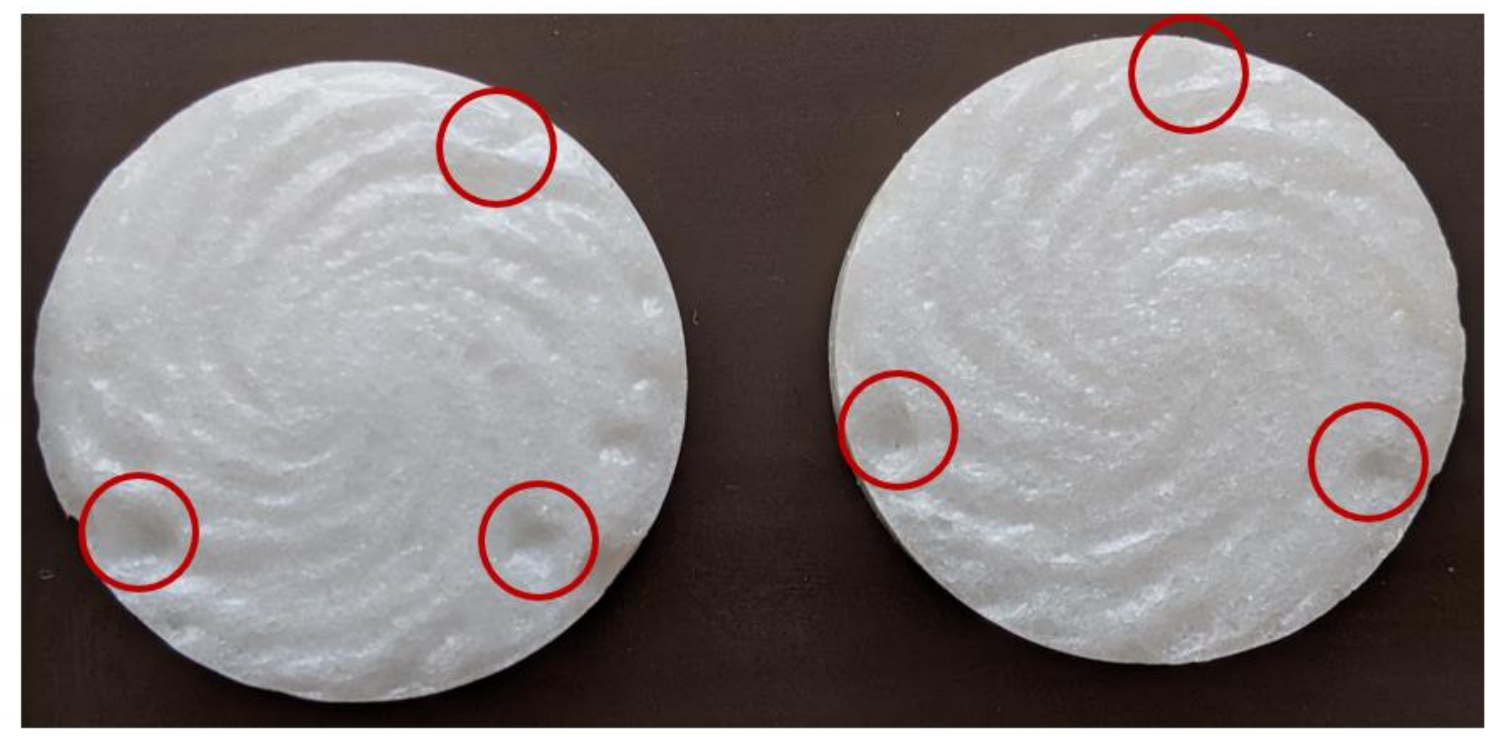

Fig. III-6 1.46 in. disks after duplicate RDA tests at 587 rpm. Reprinted with permission from (Ivanshin et al. 2021).

Fig. III-6 shows two 1.46 in. diameter disks after duplicate tests at $587 \mathrm{rpm}$. The cavities on the outer region of both disks are located at an equal radial distance from the center of rotation and arranged in a similar pattern.

Previous studies on the hydrodynamic boundary layer of an RD are mostly limited to flow transition over smooth disks, with no dissolution of their surface. Nevertheless, the features similar to those observed in the present study are reported. The flow instabilities with stationary spiral patterns (vortices) appear on the outer region of the disk surface as soon as the transition from laminar to turbulent regimes starts. An important feature of the spiral vortices is that they are fixed relative to the surface of the disk, i.e., have no phase velocity. Kohama (1984) observed the formation of ring-like vortices on the surface of spiral vortices just before the transition to the turbulent regime (Fig. III-7a). Although in Kohama's tests the disk surface was not dissolving, one can mention similarities between 
the visualized boundary layer flow (Kohama, 1984, Fig. III-7a) and the appearance of the disks after the RDA tests (Fig. III-3 and Fig. III-8). In both cases, the smooth central region of laminar flow, thin ring-like area of transition flow occupied by spiral vortices, and outer region of turbulent flow with an irregular pattern are present.
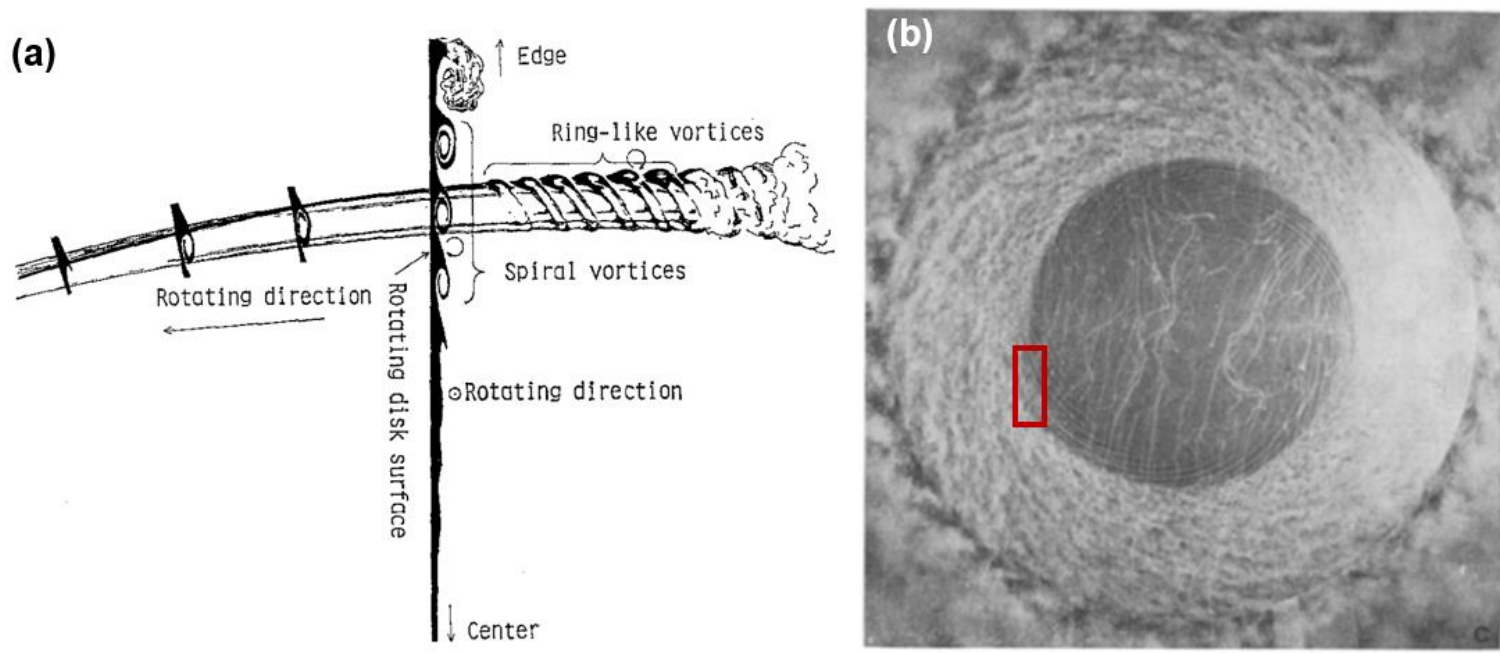

Fig. III-7 Transition from laminar to turbulent regime in boundary layer flow: (a) schematic structure of the transition regime with spiral and ring-like vortices, (b) the photograph showing three regions on the surface of the disk - laminar in the center, transition with many spiral vortices, and turbulent with irregular flow pattern (after Kohama 1984). Reprinted with permission from (Ivanshin et al. 2021). 


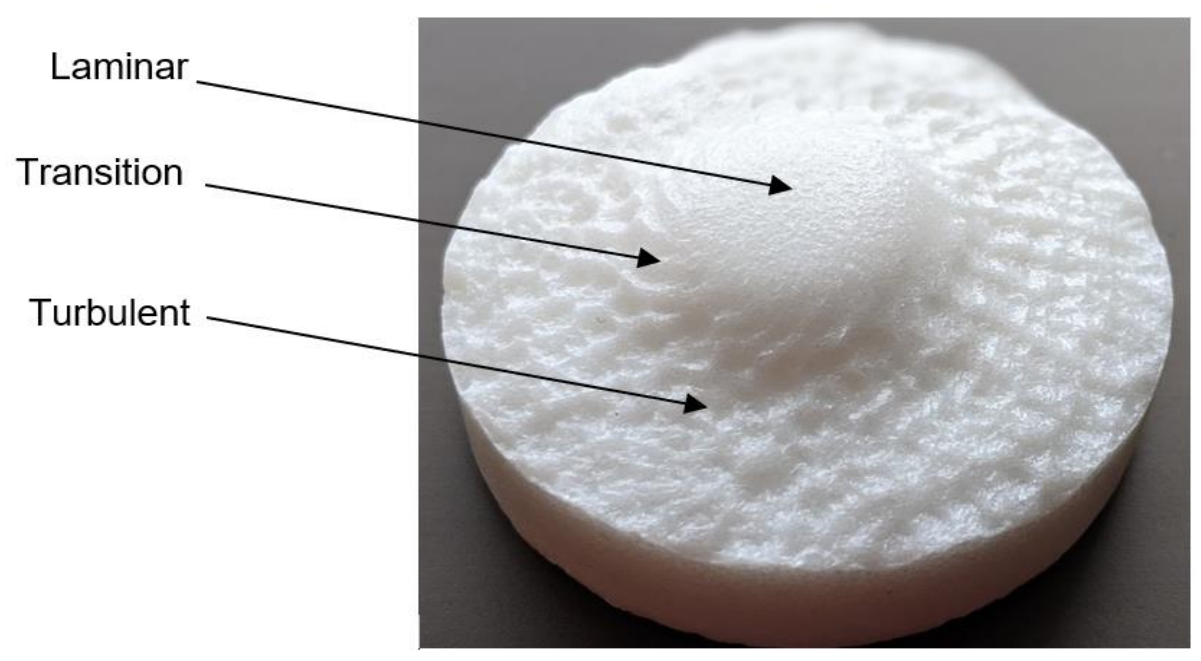

Fig. III-8 1.46 in. disk after the dissolution test showing the regions of laminar, transition, and turbulent flow regimes in the boundary layer. Note the similarity with Fig. 6(b). Reprinted with permission from (Ivanshin et al. 2021).

It is suggested in the present work that cavities are formed on the surfaces of the disks because of an increase in the local mass transfer during the transition to turbulent flow in the boundary layer. The mechanism can be similar to that reported by Kohama (1984), namely the formation and breakdown of the ring-like vortices on the surface of the spiral vortices. Once a certain value of the Reynolds number is passed, these hydrodynamic instabilities make the disk surface non-uniformly accessible.

Although turbulence progresses with dissolution, the flow instabilities increase the local mass transfer to the surface of the disk from the beginning of the tests where cavities are observed. The representative data in Fig. III-9 confirms this statement. Even for the test at 1,555 rpm-with the most dissolution-the straight line with a correlation coefficient of 0.994 fits the data points 1-4 used in calculations. Moreover, calcium ion concentration 
in the fifth acid sample is just slightly above the trendline. Therefore, it was concluded that the evolution of the surface area because of the formation of the irregular etched pattern has a subordinate effect on the overall increase of the mass-transfer rate.

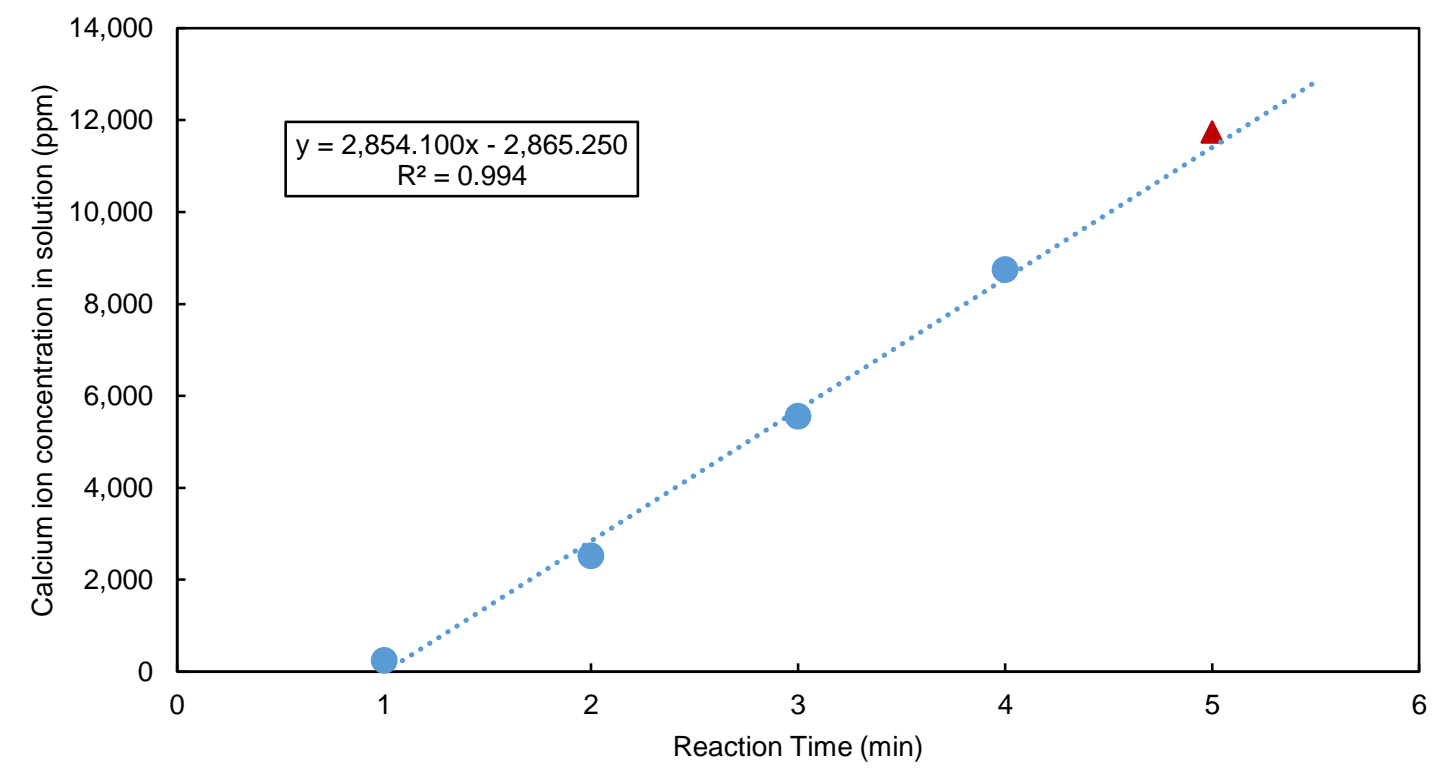

Fig. III-9 Concentration of calcium ion in the fluid samples withdrawn during the RDA test at $1,555 \mathrm{rpm}$ using the disk of 1.46 in. diameter. The first four data points were used to calculate the mass-transfer rate to the disk surface. Reprinted with permission from (Ivanshin et al. 2021).

The trendlines presented on Fig. III-2 are then replotted using only the data points from the experiments that do not result in the formation of cavities on the surface of the disks (Fig. III-10, filled markers). The trendlines for 0.72 and 1.11 in. disks become almost parallel, while the trendline for 1.46 in. disk still slightly deviates. Recalculated diffusion coefficients become equal $3.32 \times 10^{-5}$ and $4.30 \times 10^{-5} \mathrm{~cm}^{2} / \mathrm{s}$ for 1.11 , and 1.46 in. disks, respectively. The former value being close to $3.08 \times 10^{-5} \mathrm{~cm}^{2} / \mathrm{s}$ calculated earlier using the data from the tests with 0.72 in. disks. 


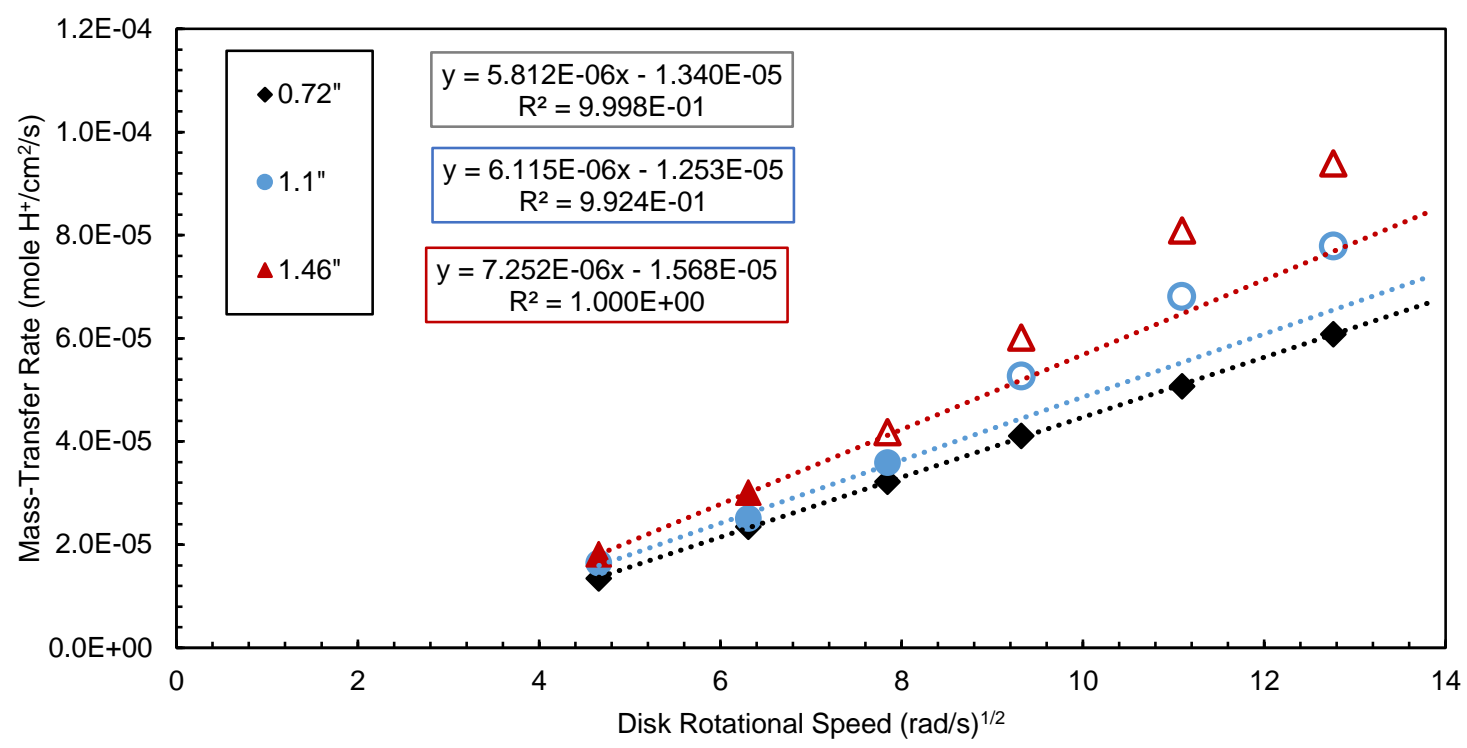

Fig. III-10 Mass-transfer rate to the disks of calcite marble as a function of the disk rotational speed in $15 \mathrm{wt} \% \mathrm{HCl}$ at $100^{\circ} \mathrm{F}$. Trendlines including only the filled markers, i.e. the data points in laminar flow regime. Reprinted with permission from (Ivanshin et al. 2021).

In order to mimic the reservoir conditions, RDA tests are commonly run at temperatures higher than $100^{\circ} \mathrm{F}$. Although not reported here, it was observed that the non-uniform accessibility of the surface of 1.46 in. diameter disks when dissolving them in lower reactivity acid systems. For example, calcites of sedimentary origin in:

- Organic acids at $176^{\circ} \mathrm{F}$ and disk rotational speeds starting from $587 \mathrm{rpm}$. Calculation including the data points in the turbulent regime would overestimate the acid-diffusion coefficient 88 times.

- Emulsified $15 \mathrm{wt} \% \mathrm{HCl}$ at $250-275^{\circ} \mathrm{F}$ and disk rotational speeds starting from $1,200 \mathrm{rpm}$.

- Gelled $15 \mathrm{wt} \% \mathrm{HCl}$ at $275^{\circ} \mathrm{F}$ and disk rotational speeds starting from $480 \mathrm{rpm}$.

Also, dolomites dissolve in $15 \mathrm{wt} \% \mathrm{HCl}$ at $180^{\circ} \mathrm{F}$ and disk rotational speeds starting from $311 \mathrm{rpm}$. 
In recent publications reporting RDA tests, calcite disks of 1.5 in. diameter were dissolved at temperatures up to $300^{\circ} \mathrm{F}$ and rotational speeds up to $1,800 \mathrm{rpm}$. The slope of the presented plots of the dissolution rate over the square root of the disk rotational speed increases, usually at 400-800 rpm. As such, the calculations using all the experimental data points result in overestimation of the acid-diffusion coefficient.

The spiral patterns on the surface of the disk have been reported from the study of the electrochemical dissolution processes of metals using RDE. Baune (2002) was dissolving disks of $10 \mathrm{~mm}$ diameter in $3.5 \mathrm{M} \mathrm{FeCl}_{3}$ solution while maintaining a rotational speed of up to $5,000 \mathrm{rpm}$, or $\mathrm{Re} \leq 1.2 \times 10^{4}$. The coupling of the hydrodynamic (spiral vortices) and dissolution processes led to the formation of a pattern on the surface of the RD (Baune 2002, their Figs. 5.3 and 5.4) that is similar to observations in the present study at moderate rotational speeds. Increasing rotational speed forced a shift and an intensification of convection vortices, leading to a higher dissolution rate, stronger pattern formation and the onset of spiral patterns closer to the center. No formation of cavities on the surfaces of disks was reported. However, compared to the present study, Baune (2002) did not reach $\mathrm{Re}_{\mathrm{cr}}$, and disk electrodes were dissolving more slowly than calcite disks in the present study. Thus, it was decided to perform the RDA tests at lower dissolution rates by decreasing the concentration of acid solution and experiment temperature.

\section{Experimental Dissolution in $1 \mathrm{~N} \mathrm{HCl}$ at $73.4^{\circ} \mathrm{F}$}

The experiments presented in this section are regarded as a "model experiment." The reason being is that usually: (1) reservoir temperatures are higher than $73.4^{\circ} \mathrm{F}$, and (2) 
more reactive acids are used for stimulation. These experiments are aimed to determine if the uniform disk accessibility is maintained:

1. At lower dissolution rates, since the development of an etching pattern or generation of $\mathrm{CO}_{2}$ can promote turbulence.

2. In the rocks with smaller grains, since sedimentary rocks are ordinarily composed of $<1000 \mu \mathrm{m}$ grains.

Two important regions close to the surface of the RD are the hydrodynamic and diffusion boundary layers. Within the hydrodynamic boundary layer, the radial and tangential velocity components are not zero, while beyond that layer, only axial fluid motion exists. The diffusion boundary layer is the region close to the solid-liquid interface where the acid concentration changes rapidly and the concentration gradient is at a maximum. While the diffusion boundary layer has no clearly defined boundary, according to Levich (1962) its thickness for the usual values of diffusion coefficient $\mathrm{D} \sim 10^{-5} \mathrm{~cm}^{2} / \mathrm{s}$ in water and for $v$ $\sim 10^{-2} \mathrm{~cm}^{2} / \mathrm{s}$ constitutes only $5 \%$ of the thickness of the hydrodynamic boundary layer. Eqs. 3 and 4 (Levich 1962) show that the thickness of the hydrodynamic boundary layer depends on the physical properties and the velocity of the solution, while the thickness of the diffusion layer also depends on the diffusion coefficient:

$$
\begin{aligned}
& \delta_{0}=3.6 \sqrt{\frac{v}{\omega}}, \ldots \ldots \ldots \ldots \ldots \ldots \ldots \ldots \ldots \ldots \ldots \ldots \ldots \ldots \\
& \delta^{\prime}=\frac{D C_{b}}{j}=1.61\left(\frac{D}{v}\right)^{1 / 3} \sqrt{\frac{v}{\omega}} \approx 0.5\left(\frac{D}{v}\right)^{\frac{1}{3}} \delta_{0}
\end{aligned}
$$


where $\delta_{0}$ is the thickness of the hydrodynamic boundary layer, and $\delta^{\prime}$ is the thickness of the diffusion boundary layer.

Table III-2 shows the thicknesses of the hydrodynamic and diffusion boundary layers calculated using Eqs. 3 and 4 and the diffusion coefficient of $3.08 \times 10^{-5} \mathrm{~cm}^{2} / \mathrm{s}$. If $1000 \mu \mathrm{m}$ grains composing calcite marble are half-protruding from its surface is assumed, then at low disk rotational speeds, the hydrodynamic boundary layer extends above the grains. As the hydrodynamic boundary layer thins out with the increase of the disk rotational speed, the presence of grains progressively affects the radial and tangential motion of fluid in the vicinity of the disk, promoting a transition to turbulence. The effect is especially pronounced at the highest disk rotational speed, where the hydrodynamic boundary layer is almost twice thinner than the half-grain (Table III-2).

\begin{tabular}{cccc}
\hline \multicolumn{2}{c}{ Rotational Speed } & \multicolumn{2}{c}{$\begin{array}{c}\text { Thickness of Boundary } \\
\text { Layer, } \mu \mathrm{m}\end{array}$} \\
$\mathrm{rpm}$ & $\mathrm{rad} / \mathrm{s}$ & Hydrodynamic & Diffusion \\
\hline 207 & 21.7 & 823.2 & 57.4 \\
380 & 39.8 & 608.0 & 42.4 \\
588 & 61.5 & 489.1 & 34.1 \\
829 & 86.9 & 411.6 & 28.7 \\
1,175 & 123.0 & 345.8 & 24.1 \\
1,555 & 162.9 & 300.6 & 21.0 \\
\hline
\end{tabular}

Table III-2 Thicknesses of the hydrodynamic and diffusion boundary layers. Reprinted with permission from (Ivanshin et al. 2021). 
The 1.46 in. diameter disks were cut from two blocks of calcite marble. One-used in the previous tests-was composed of grains with a maximum size of approximately $1000 \mu \mathrm{m}$, while the other contained grains of $150 \mu \mathrm{m}$. In the latter case, the hydrodynamic boundary layer will be entirely covering the grains even at the highest disk rotational speed. The dissolution tests were run at 518 and 1,175 rpm. The lower value was chosen as at this disk rotational speed, the laminar flow was maintained in the tests described in the previous section, and the thickness of the hydrodynamic boundary layer is close to the half-size of the $1000 \mu \mathrm{m}$ grains (Table III-2).

Fig. III-11 and Fig. III-12 show the well-defined etched lines on the disks of both grain sizes after the dissolution at $518 \mathrm{rpm}$. After the tests at 1,175 rpm, the etched lines are easier to distinguish on the disks composed of smaller grains. Nevertheless, they disappear close to the edge, where the surface is covered with small cavities. Although the uniform accessibility of the surface is lost at high disk rotational speed, the rate of mass transfer is in good agreement with Levich's (1962) theory (Fig. III-13). The data points for both rocks fall on the straight line passing through the origin. The mass-transfer rate to the disks composed of smaller grains is slightly higher, which is explained by the larger reactive surface area. The calculated diffusion coefficients are $4.28 \times 10^{-5}$ and $4.69 \times 10^{-5} \mathrm{~cm}^{2} / \mathrm{s}$ for the disks composed of 1000 and $150 \mu \mathrm{m}$ grains, respectively. These values are in good agreement with $3.97 \times 10^{-5} \mathrm{~cm}^{2} / \mathrm{s}$ obtained while dissolving the disks of calcite marble composed of the maximum crystal size of $2000 \mu \mathrm{m}$ at similar conditions (Lund et al. 1975). 


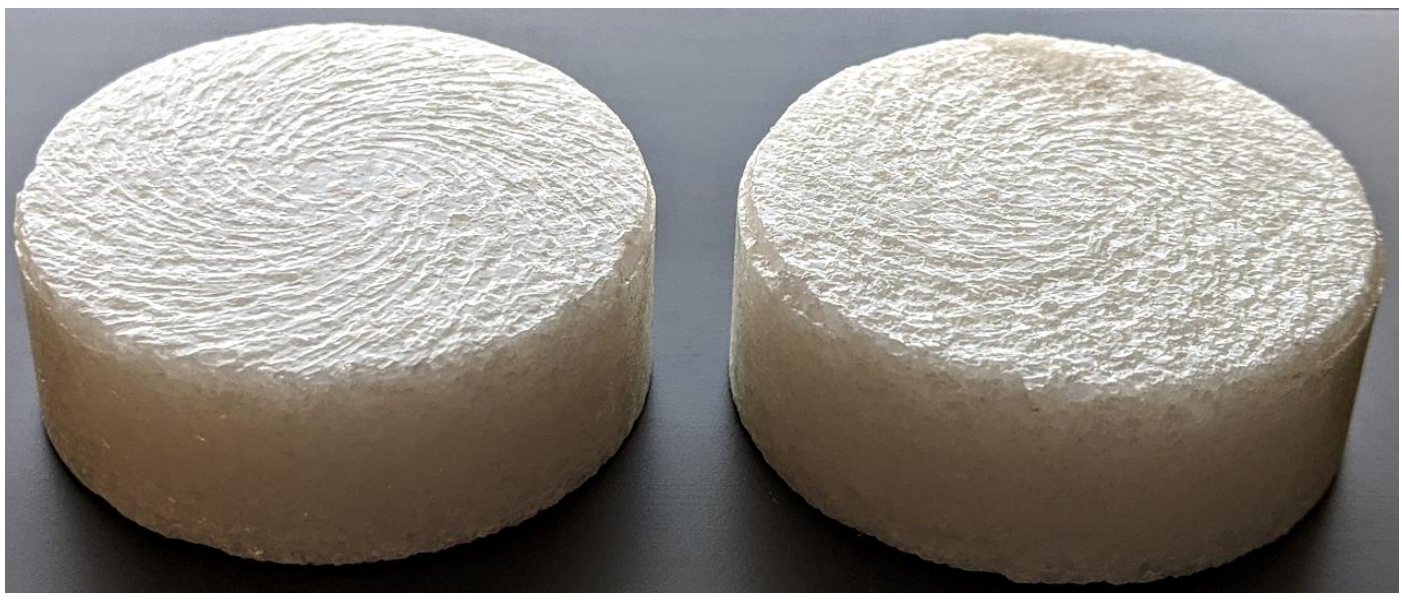

Fig. III-11 1.46 in. disks composed of grains of maximum size of $1000 \mu \mathrm{m}$ after dissolution in $1 \mathrm{~N} \mathrm{HCl}$ at $73.4^{\circ} \mathrm{F}$, and disk rotational speeds of 518 (left) and 1,175 (right) rpm. Reprinted with permission from (Ivanshin et al. 2021).

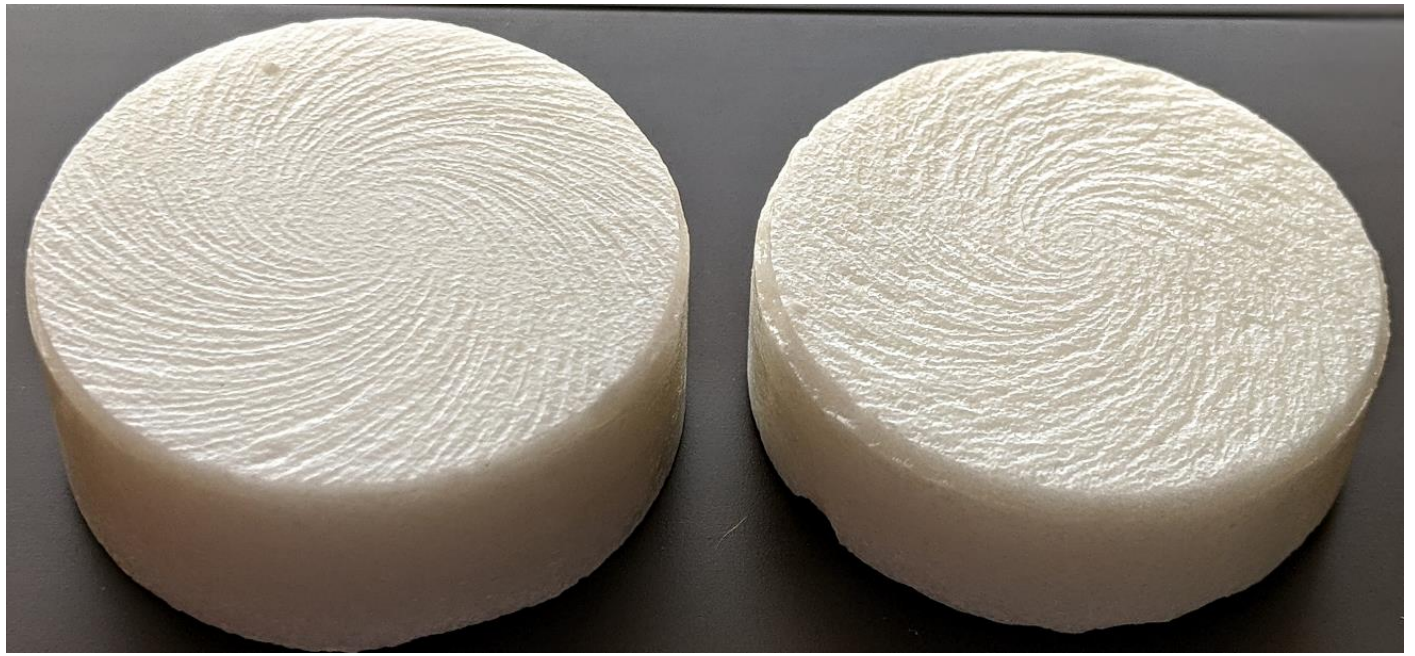

Fig. III-12 1.46 in. disks composed of grains of maximum size of $150 \mu \mathrm{m}$ after dissolution in $1 \mathrm{~N} \mathrm{HCl}$ at $73.4^{\circ} \mathrm{F}$, and disk rotational speeds of 518 (left) and 1,175 (right) rpm. Reprinted with permission from (Ivanshin et al. 2021). 


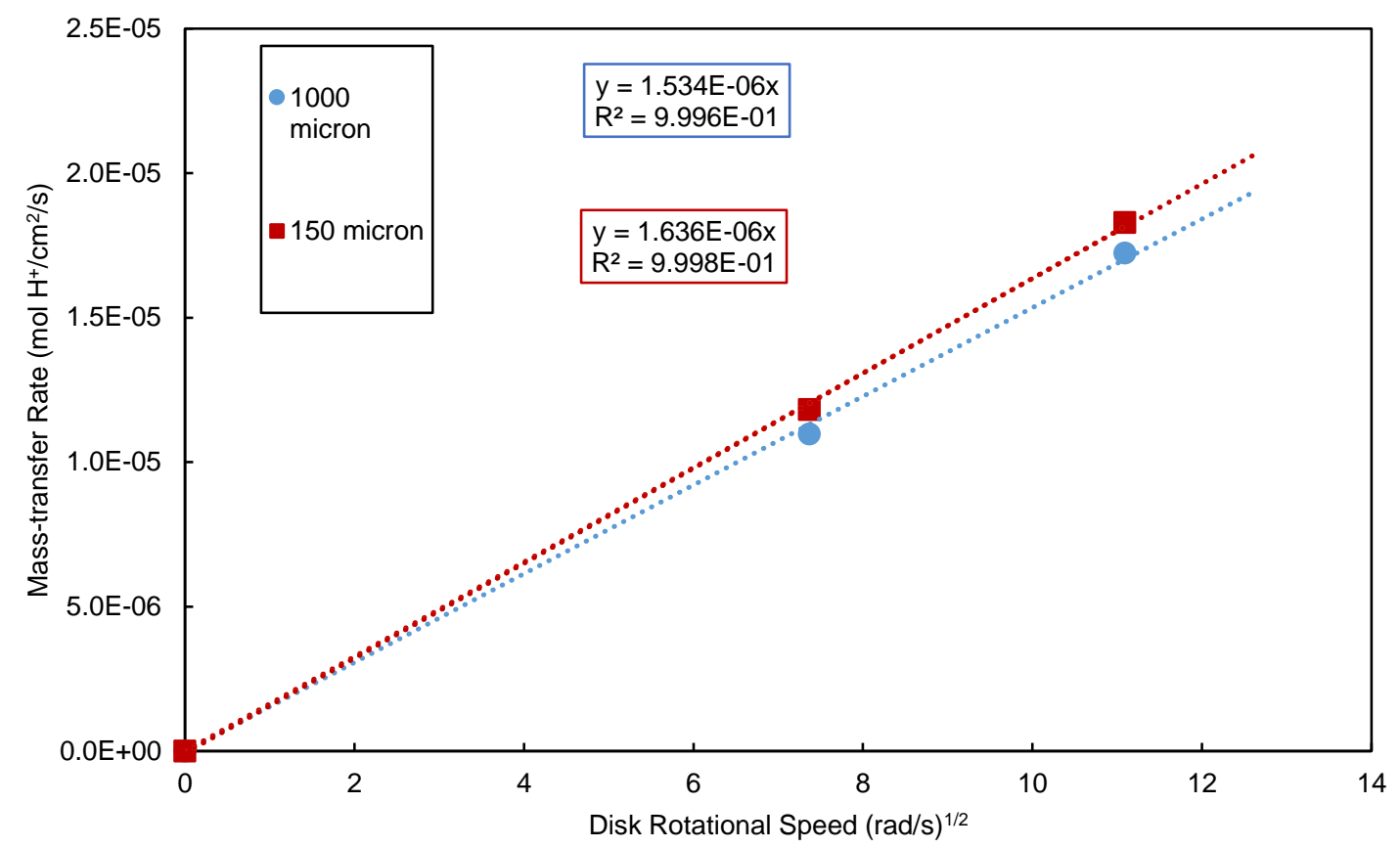

Fig. III-13 Mass-transfer rate to the disks of calcite marble as a function of the disk rotation speed in $1 \mathrm{~N} \mathrm{HCl}$ at $73.4^{\circ} \mathrm{F}$. Reprinted with permission from (Ivanshin et al. 2021).

Performed tests confirm the conclusion of Rogers and Taylor (1963): at certain experimental conditions, Levich's (1962) theory can be applied even when the disk surface becomes non-uniformly accessible, i.e., when the assumption of laminar flow is not satisfied. Although not reported here, similar results were observed when dissolving calcite marble in $25 \mathrm{wt} \%$ glutamic acid diacetic acid (GLDA) solution of $\mathrm{pH} 3.8$ at $176^{\circ} \mathrm{F}$ and the disk rotational speeds up to $1,555 \mathrm{rpm}$.

Comparing the plots on Fig. III-2 and Fig. III-13 at equal disk rotational speeds, one can note around 4.5 times higher mass-transfer rate during the tests with $15 \mathrm{wt} \% \mathrm{HCl}$ at $100^{\circ} \mathrm{F}$. 
Thus, these "certain experimental conditions" for RDA tests are low reactivity systems such as dissolution of calcite in $1 \mathrm{~N} \mathrm{HCl}$ at $73.4^{\circ} \mathrm{F}$ or $25 \mathrm{wt} \%$ GLDA at $176^{\circ} \mathrm{F}$. In the systems with the high dissolution rate, large density gradients in the hydrodynamic boundary layer-the density of the bulk solution is smaller than the density of solution at the solid-liquid interface-induce natural convection. The coupling of natural and forced convection flows promotes the transition to turbulence and increases mass transport.

In RDA experiments, the mass transfer to the surface of the disk depends on fluid flow, diffusion of the reactive species, and dissolution of the solid. The contribution of each process depends on the particular experimental conditions: temperature, properties of acid solution and rock, etc. Thus, no universal recommendations on the measurement of the acid-diffusion coefficient can be drawn from this work. The appearance of the disk after the dissolution tests should serve as a first indication of the flow regime at the disk surface. The increase in the slope of the data points on the plot of the mass-transfer rate over the square root of the disk rotational speed can provide further evidence of the loss of uniform accessibility to the disk surface.

Simulation: Impact of Disk size on the Disk Dissolution Rate and Velocity Profile

Table III-3 summarizes the slopes of the plots of the mass transfer of $\mathrm{H}^{+}$as a function of the square root of the disk rotational speed-similar to the slopes reported in Fig. III-2 and Fig. III-10-to the three simulated disks. As observed in the experiments, the slope increases with disk diameter ranging between $3.03 \times 10^{-6}$ to $4.53 \times 10^{-6} \mathrm{~mol} / \mathrm{cm}^{2} \mathrm{~s}^{1 / 2}$ for the 0.75 and 1.5 in. disks, respectively. These values are smaller than the experimental values 
obtained which can be explained by the homogeneity of the simulated disks that is not present in reality.

\begin{tabular}{cc} 
Disk Diameter (in.) & Slope $\left(\mathrm{mol} \mathrm{H}^{+} / \mathrm{cm}^{2} / \mathrm{s}^{1 / 2}\right) \times 10^{-6}$ \\
\hline 1.5 & 4.53 \\
1 & 3.70 \\
0.75 & 3.03 \\
\hline
\end{tabular}

Table III-3 Slope of the plot of rate of mass transfer of $\mathrm{H}^{+}$as a function of square root of disk rotational speed at three disk diameters showing a direct relationship between the mass transfer of $\mathrm{H}^{+}$and disk diameter. Reprinted with permission from (Ivanshin et al. 2021).

In order to explain the increase in slope with increasing disk diameter, the velocity profiles under the disks were studied. Fig. III-14 shows the velocity profiles below the disks of the three diameters at 209 and 1,200 rpm at five minutes. As the disk size increases, the velocity magnitude increases, which results in an increased disk dissolution rate. The maximum velocity magnitude at 1,200 rpm ranges between 117 and $230 \mathrm{~cm} / \mathrm{s}$ on the 0.75 and 1.5 in. disks, respectively. For the 0.75 in. disk, axisymmetric flow can be observed under the disk for the two rotational speeds for the full duration of the test, indicating laminar flow. At 1,200 rpm, the velocity under the 0.75 in. disk can be seen transitioning from axisymmetric flow as more vortices are generated in the region between the side of the disk and the boundary of the reactor. A similar velocity pattern is observed in the $1 \mathrm{in}$. disk case at $209 \mathrm{rpm}$. However, at 1,200 rpm for the $1 \mathrm{in}$. disk and at both rotational speeds for the 1.5 in. disks, the velocity profile is asymmetric. Although not reported here, the velocity profile for these cases is changing with time. It can also be observed that at the 
extreme condition of 1,200 rpm and 1.5 in. disk, the fluid is creating more vortices under the disk.

These result show that turbulence starts at a lower Reynolds number in contrast to what is commonly assumed in the literature and that the disk is non-uniformly accessible by the acid.

A. 0.75 in. disk at 209 rpm

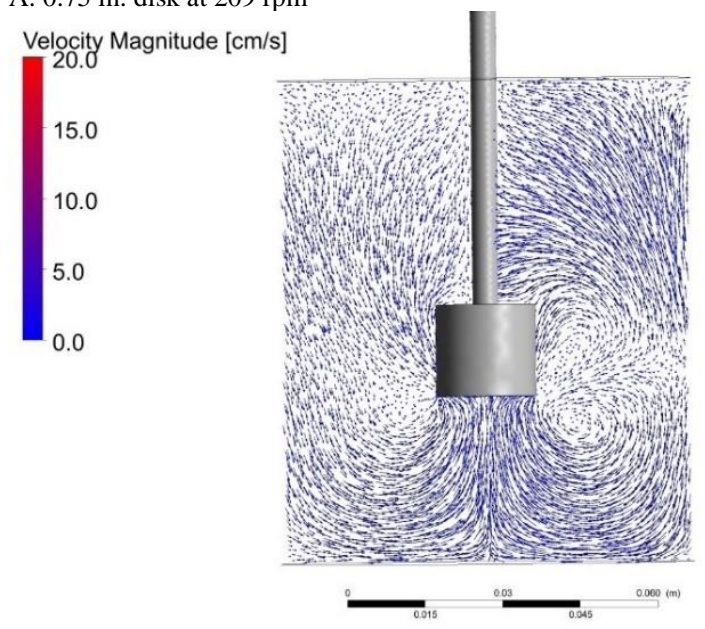

C. 1 in. disk at $209 \mathrm{rpm}$ Velocity Magnitude $[\mathrm{cm} / \mathrm{s}]$ 20.3 13.5 6.8 0.0

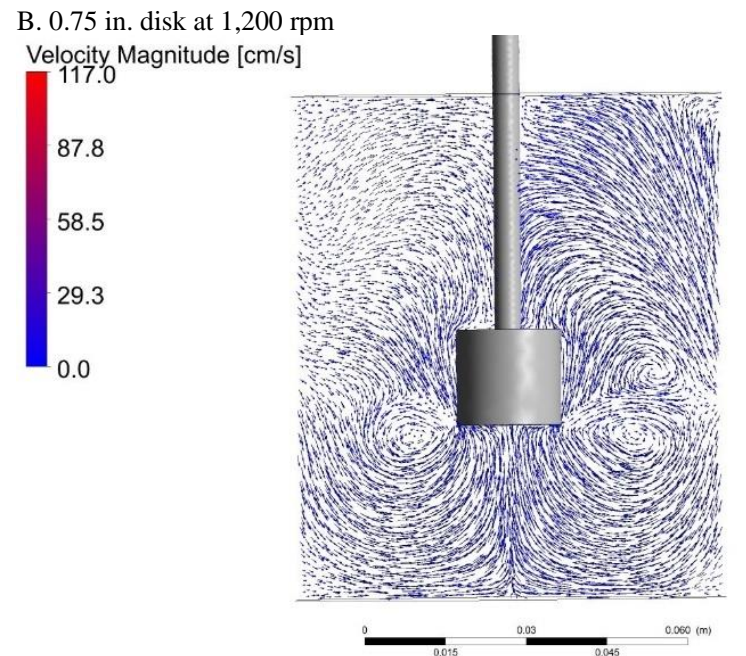

D. 1 in. disk at $1,200 \mathrm{rpm}$

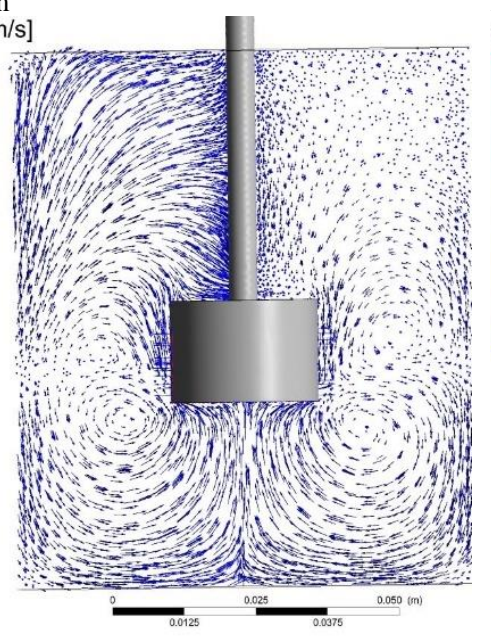
Velocity Magnitude $[\mathrm{cm} / \mathrm{s}]$

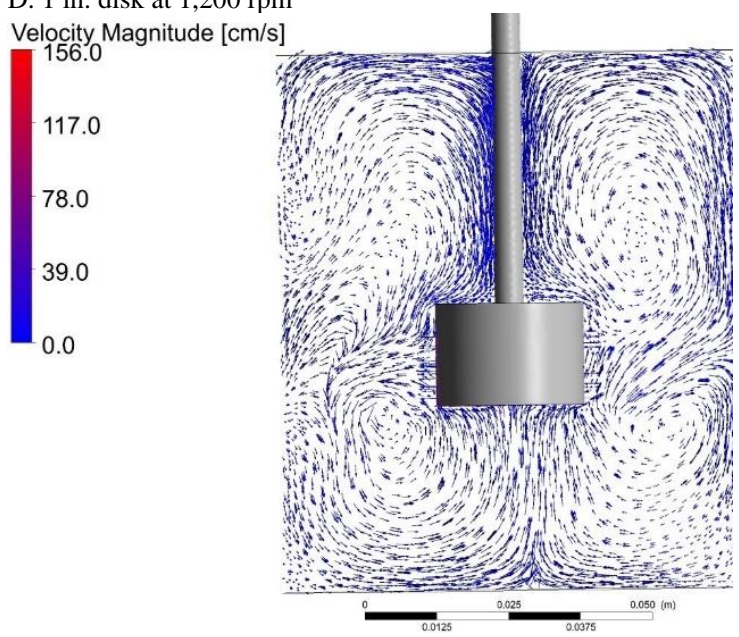

Fig. III-14 Side view of the disks comparing the velocity profiles at different disk size and rotational speed at five minutes. Dissolution in $15 \mathrm{wt} \% \mathrm{HCl}$ at $100^{\circ} \mathrm{F}$ was simulated. Reprinted with permission from (Ivanshin et al. 2021). 
E. 1.5 in. disk at $209 \mathrm{rpm}$

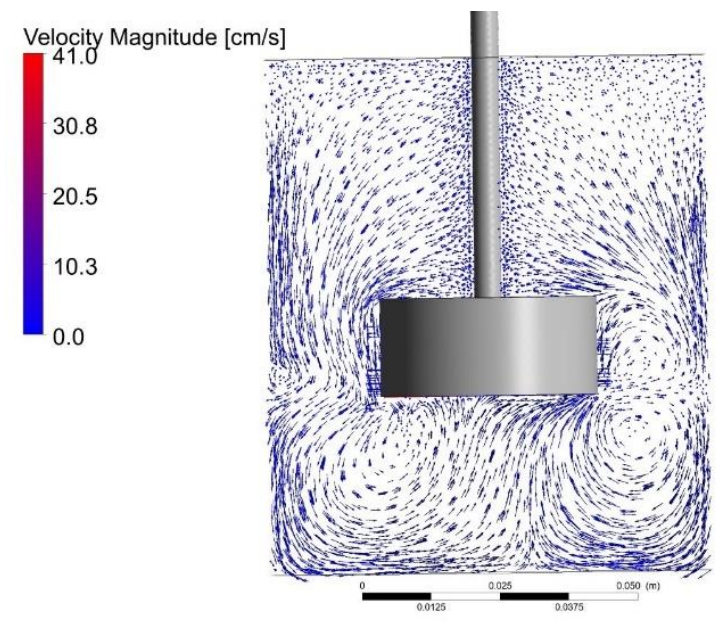

F. 1.5 in. disk at 1,200 rpm

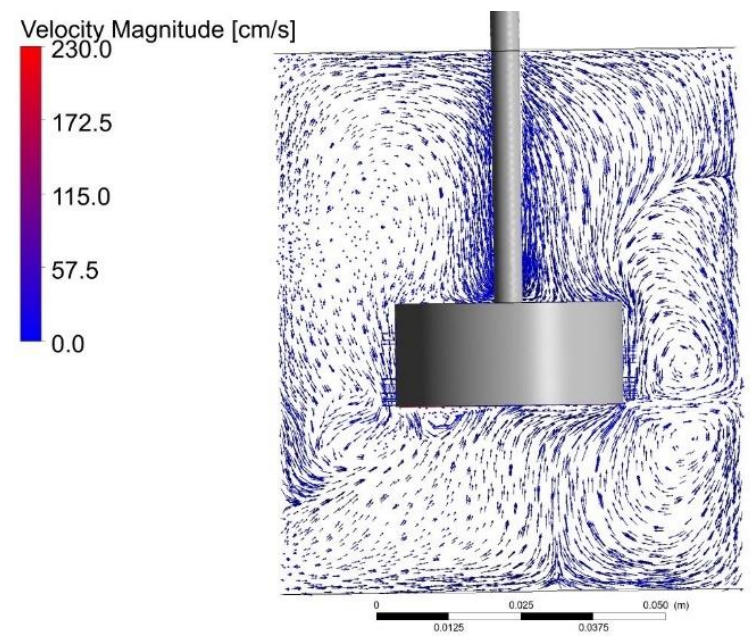

Fig. III 14 Continued. 


\section{CHAPTER IV}

\section{A CALIBRATED COMPUTATIONAL FLUID DYNAMICS MODEL FOR SIMULATING THE ROTATING DISK APPARATUS*}

\section{Method}

\section{Experimental Studies}

The RDA used in this work is shown in Fig. IV-1. Acid was mixed with 1 wt $\%$ corrosion inhibitor using a magnetic stirrer. Acid density and viscosity were measured at temperatures up to $185^{\circ} \mathrm{F}$ using a density meter and a capillary viscometer, respectively. The values of density and viscosity at higher temperatures were extrapolated from these values. Fig. IV-2 and Fig. IV-3 show the density and viscosity results, respectively, for the $\mathrm{HCl}$ used in this work.

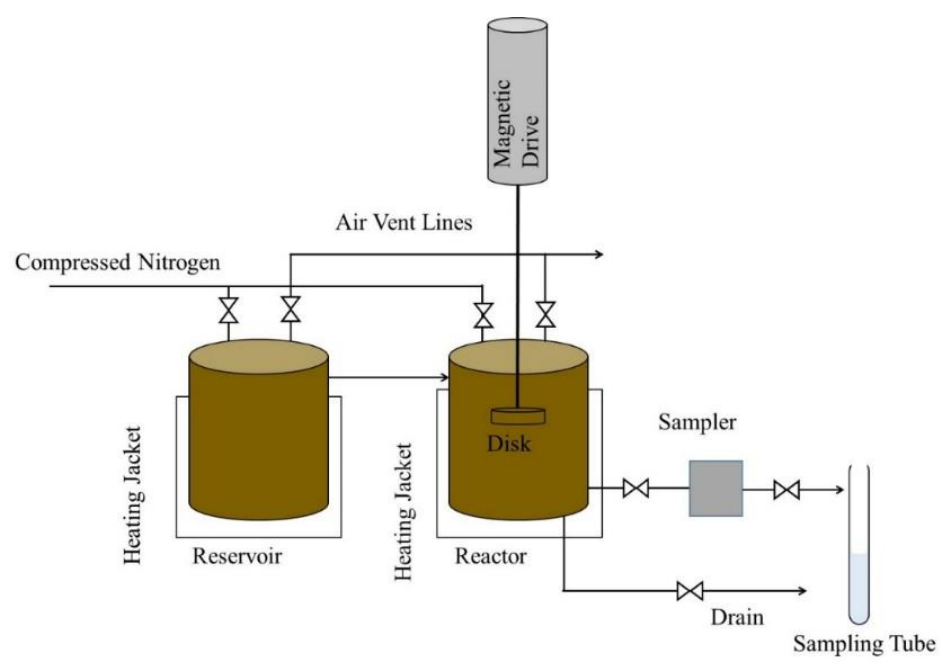

Fig. IV-1 Schematic for the rotating disk apparatus. Reprinted with permission from (Kotb et al. 2021).

\footnotetext{
* Part of this chapter is reprinted with permission from "A Calibrated Computational Fluid Dynamics Model for Simulating the Rotating Disk Apparatus" by Kotb, A. et al. 2021, SPE J, Copyright 2021 by Society of Petroleum Engineers.
} 


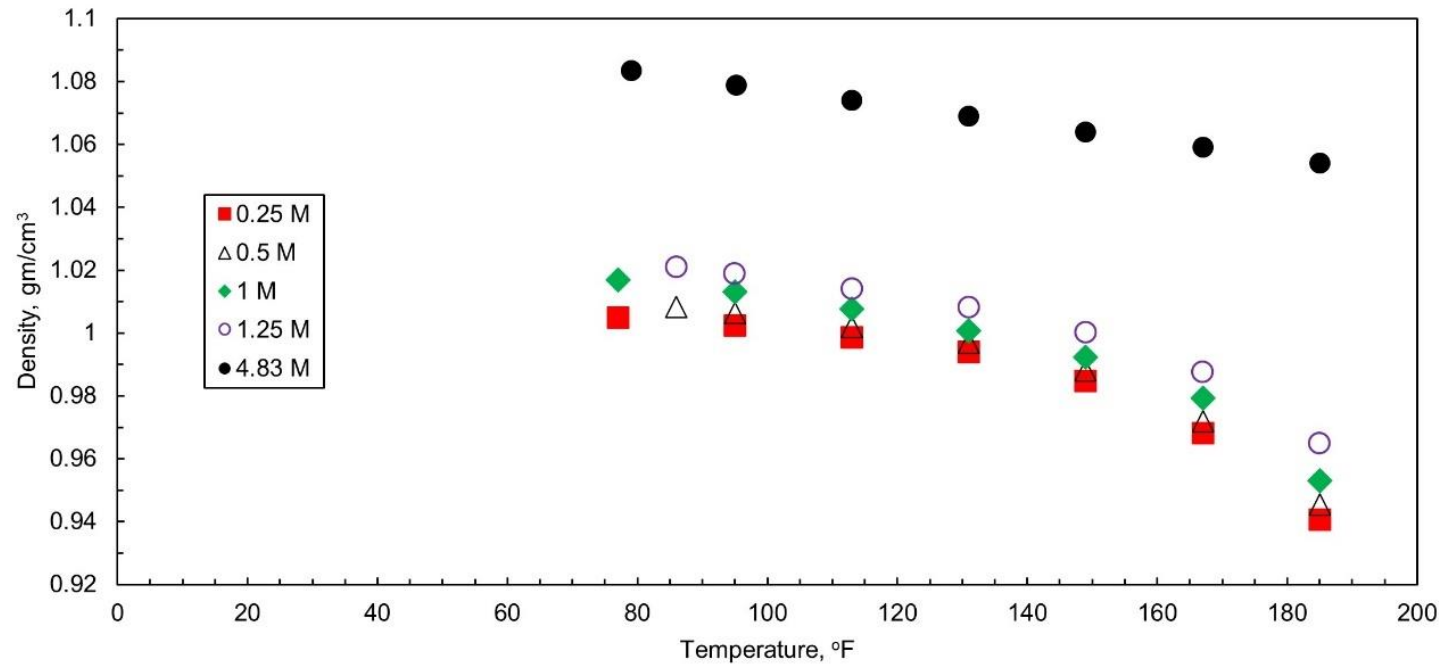

Fig. IV-2 Density data for different molarity HCl. All Acids contained 1 wt\% corrosion inhibitor. Reprinted with permission from (Kotb et al. 2021).

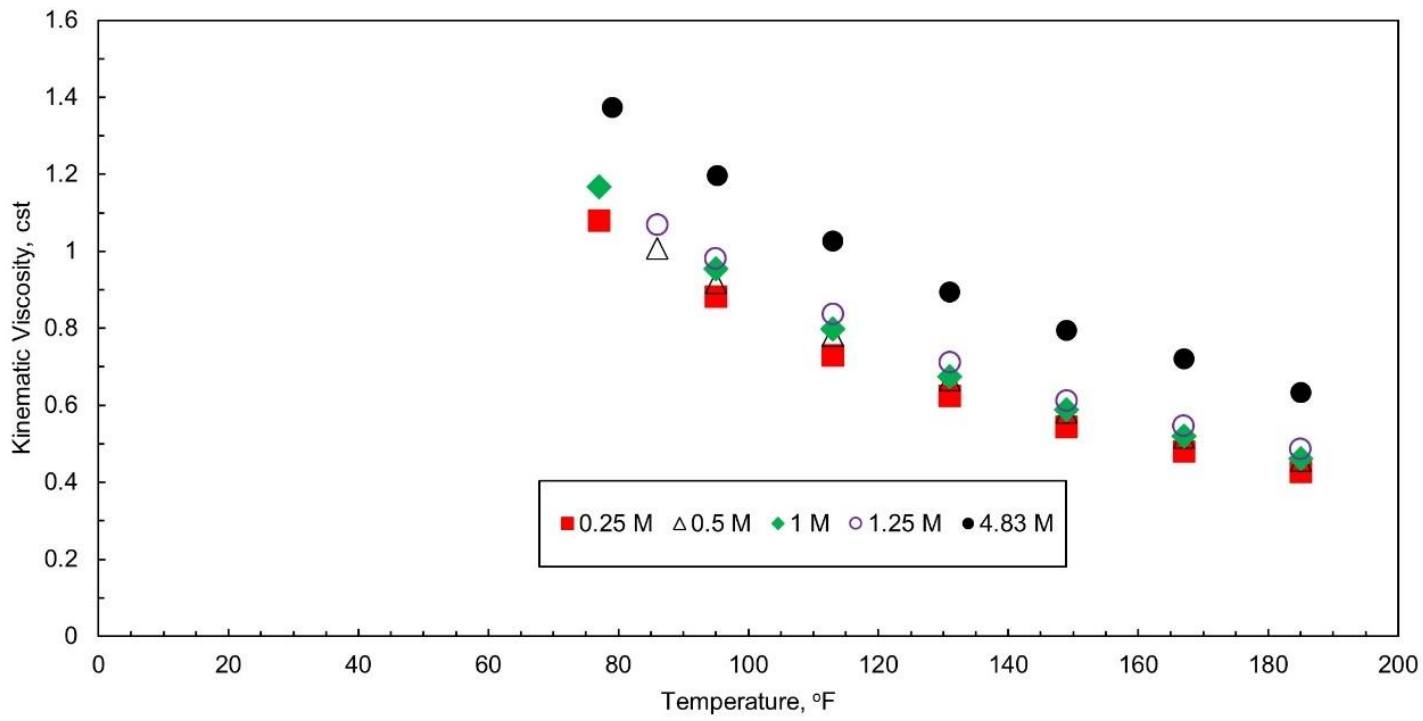

Fig. IV-3 Kinematic viscosity data for different molarity HCl. All Acids contained 1 wt\% corrosion inhibitor. Reprinted with permission from (Kotb et al. 2021). 
The procedure outlined by Fredd (1997) was followed for running tests. A disk with 1.5 in. diameter and $0.5 \mathrm{in}$. thickness was heated to $220^{\circ} \mathrm{F}$ to evaporate water and was then weighed. Next, sandpaper was used to smooth the sides of the disk. The disk was further soaked in $0.1 \mathrm{M} \mathrm{HCl}$ for 30 minutes to remove fines and was then cleaned with deionized water. It was then laminated with a shrinkable nonstick coating such that only one surface is exposed to the acid. The disk was then immersed in the reactor and connected to a magnetic drive for rotation. $\mathrm{HCl}\left(450 \mathrm{~cm}^{3}\right.$ volume $)$ was heated and pressurized to 1,500 psi in the reservoir. The acid was then transported to the reactor - a process that takes 10$12 \mathrm{~s}$, after which the reservoir was pressurized to $1,500 \mathrm{psi}$, and disk rotation started. The pressure keeps $\mathrm{CO}_{2}$ in solution and maintains a single-phase fluid (Welton and Domelen 2008; Nasr-El-Din et al. 2008; Taylor and Nasr-El-Din 2009). Acid samples were taken from the reactor at 1-minute intervals over the test duration. Calcium concentration in these samples was measured using inductively coupled plasma (ICP). This concentration, coupled with the acid properties, was obtained from four experiments performed at different disk rotational speeds to calculate the diffusion coefficient using Newman's equation (1966). The disk was dried and weighed after the test to determine the amount of rock dissolved during the reaction.

\section{Simulation Studies}

The two-scale (averaged-continuum) approach was used in the proposed CFD model because the acid transport and acid-rock reaction are modeled as an interaction between the Darcy-scale and the pore-scale. The model is also based on solving the equations of fully coupled fluid flow, reactant transport, and rock-fluid reactions. This includes the 
effect of rock dissolution on porosity and permeability change in the pore-scale due to the mineral-acid reaction. The permeability in this model is calculated using the KozenyCarman correlation as discussed by Panga et al. (2005), which used porosity to calculate permeability. The commercial software ANSYS Fluent was used to solve the masscontinuity, momentum, and transport equations using the finite volume method. The details of the model used are discussed in detail by Zhang et al. (2014) and Ali et al. (2019). For the current model, the reactor was simulated using 1,150,000 gridblocks. Information about the reactor sizing, grid independence test, and model development is discussed in Kotb and Nasr-El-Din (2020). The reactor is filled with acid, and disk rotation starts at time $=0$. A porosity value is assigned to the disk. At each timestep, porosity and permeability distributions are updated. Boundary conditions in the model were set to interior for the disk and acid with no-slip boundary condition on the disk surface, and were set to wall for the reactor boundaries and disk holder boundaries. The pressure velocity coupling used was the semi-implicit method for the pressure-linked equations which is recommended for steady state cases with some complexities as present in this case (Jang et al. 1986). The software then checks for convergence or performs additional iterations before moving to the next timestep. Convergence is achieved when the residual from continuity and velocity drops below 0.001 . This procedure continues until the end of the test duration. The simulations are run on a high-performance computing facility, which provides parallel processing capabilities that divide the model into different parts that are solved simultaneously. The computational time can take up to 3 days. 
To develop the RDA CFD model, first, 32 lab experiments were performed. Table IV-1 summarizes the conditions where the tests were performed. Outcrop Indiana limestone samples were used in 16 experiments as they have shown good agreement with field samples (Rabie et al. 2014). The 32 disks were then dried and weighed. From the disk's final mass, the amount of rock dissolved is obtained, which is referred to as $y^{p} \in[0,1]$, where the superscript $p$ denotes a response from a physical experiment to differentiate it from the observation obtained from the CFD model. Occasionally, the response from the RDA experiment is denoted as $y^{p}\left(\Phi, \omega, C_{b}, v\right)$ to reflect its functional dependence on the initial porosity $\Phi$, bulk concentration $C_{b}$, kinematic viscosity $v$, and disk rotational speed $\omega$. 


\begin{tabular}{|c|c|c|c|c|c|c|}
\hline $\begin{array}{l}\text { Rock } \\
\text { Type }\end{array}$ & $\begin{array}{c}\text { Temperature } \\
\left({ }^{\circ} \mathrm{F}\right)\end{array}$ & $\begin{array}{c}\text { Initial } \\
\text { Porosity } \\
(\mathrm{wt} \%)\end{array}$ & $\begin{array}{c}\text { Test } \\
\text { Duration } \\
(\mathrm{Min}) \\
\end{array}$ & $\begin{array}{c}\mathrm{HCl} \\
\text { Concentration } \\
(\mathrm{M}) \\
\end{array}$ & $\begin{array}{c}\text { Rotational } \\
\text { Speed } \\
(\mathrm{rpm})\end{array}$ & $\begin{array}{c}\text { Rock } \\
\text { Dissolved } \\
(\mathrm{wt} \%) \\
\end{array}$ \\
\hline \multirow{16}{*}{$\begin{array}{c}\text { Indiana } \\
\text { Limestone }\end{array}$} & \multirow{4}{*}{150} & 13.4 & \multirow{16}{*}{5} & \multirow{16}{*}{4.83} & 200 & 34 \\
\hline & & 11.8 & & & 400 & 55 \\
\hline & & 13.1 & & & 600 & 71 \\
\hline & & 12.7 & & & 800 & 88 \\
\hline & \multirow{4}{*}{200} & 12.5 & & & 100 & 28 \\
\hline & & 12.5 & & & 200 & 37 \\
\hline & & 12.1 & & & 400 & 68 \\
\hline & & 12.8 & & & 600 & 88 \\
\hline & \multirow{4}{*}{250} & 12.8 & & & 100 & 29 \\
\hline & & 13.1 & & & 200 & 49 \\
\hline & & 12.9 & & & 300 & 59 \\
\hline & & 12.7 & & & 400 & 73 \\
\hline & \multirow{4}{*}{275} & 12.3 & & & 100 & 32 \\
\hline & & 13.6 & & & 200 & 46 \\
\hline & & 12.4 & & & 300 & 59 \\
\hline & & 13.0 & & & 400 & 65 \\
\hline \multirow{16}{*}{ Marble } & \multirow{16}{*}{250} & 0.0 & \multirow{16}{*}{10} & \multirow{4}{*}{0.25} & 200 & 8 \\
\hline & & 0.0 & & & 400 & 12 \\
\hline & & 0.0 & & & 600 & 15 \\
\hline & & 0.0 & & & 1000 & 16 \\
\hline & & 0.0 & & \multirow{4}{*}{0.5} & 200 & 14 \\
\hline & & 0.0 & & & 400 & 21 \\
\hline & & 0.0 & & & 600 & 25 \\
\hline & & 0.0 & & & 1000 & 28 \\
\hline & & 0.0 & & \multirow{4}{*}{1} & 200 & 24 \\
\hline & & 0.0 & & & 400 & 33 \\
\hline & & 0.0 & & & 600 & 40 \\
\hline & & 0.0 & & & 1000 & 46 \\
\hline & & 0.0 & & \multirow{4}{*}{1.25} & 200 & 23 \\
\hline & & 0.0 & & & 400 & 38 \\
\hline & & 0.0 & & & 600 & 40 \\
\hline & & 0.0 & & & 1000 & 52 \\
\hline
\end{tabular}

Table IV-1 The different tests performed in the RDA and the results obtained. Reprinted with permission from (Kotb et al. 2021). 
A six-dimensional Latin Hypercube Design (LHD) was used to generate 80 design points at which the CFD model was run. LHD is a space-filling design that ensures that the design points are uniformly spread over the domain of the input variables (Santner et al. 2003). The CFD model takes six variables as input, namely: the initial disk porosity $\phi$, bulk acid concentration $C_{b}$, kinematic viscosity $v$, disk rotational speed $\omega$, diffusion coefficient $D_{e}$, and reaction rate coefficient $k$. The output from the CFD model run is the dissolved fraction of the disk, which is denoted by $y^{c}$, where the superscript $c$ denotes a response from a CFD run. Similarly, the CFD response will be denoted as $y^{c}\left(\phi, \omega, C_{b}, v, D_{e}, k\right)$ to reflect its functional dependence on these six inputs. Fig. IV-4 through Fig. IV-9 illustrate the design points for $C_{b}, \omega, D_{e}, k, v$, and $\phi$ for the 80 simulations performed. The values used for $\phi, C_{b}, v$, and $\omega$ were based on the experiments performed. However, the values for $D_{e}$ and $k$ used in the LHD were estimated based on the literature (Lund et al. 1975; Kung 1998; Conway et al. 1999; Gledhill and Morse 2006; Finneran and Morse 2009; Rabie et al. 2014; Peng et al. 2015). 


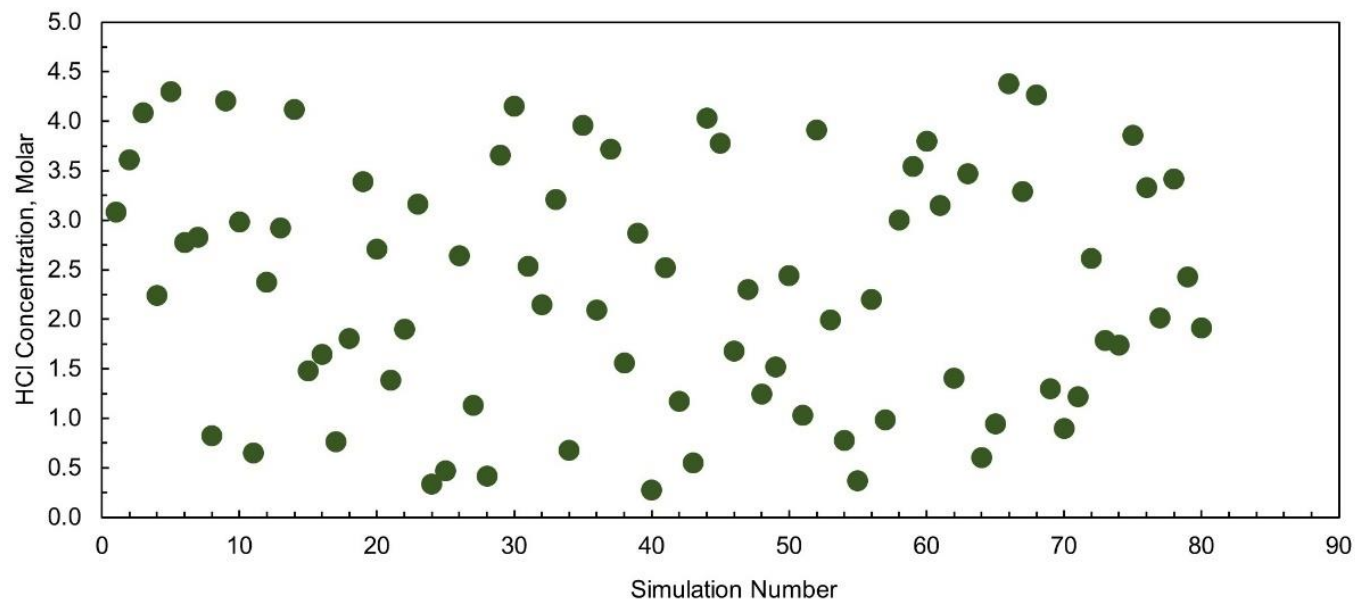

Fig. IV-4 HCl concentration used for the LHD ranged from 0.27 to $4.38 \mathrm{M}$. Reprinted with permission from (Kotb et al. 2021).

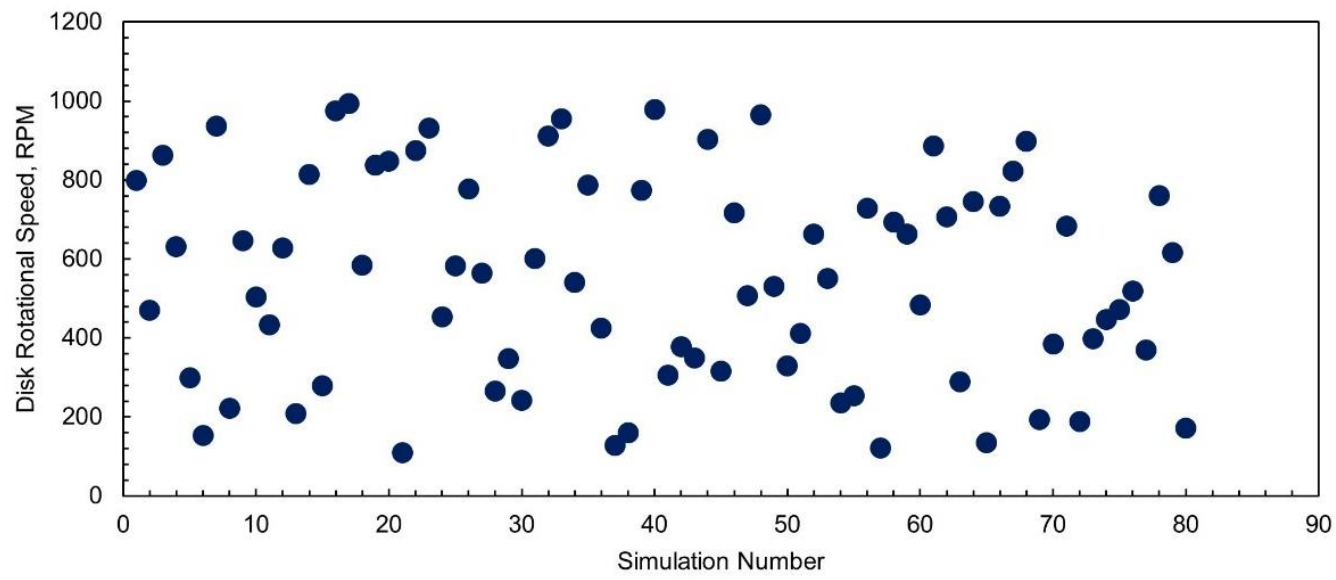

Fig. IV-5 Disk rotational speed used for the LHD ranged from 110 to $993 \mathrm{rpm}$. Reprinted with permission from (Kotb et al. 2021). 


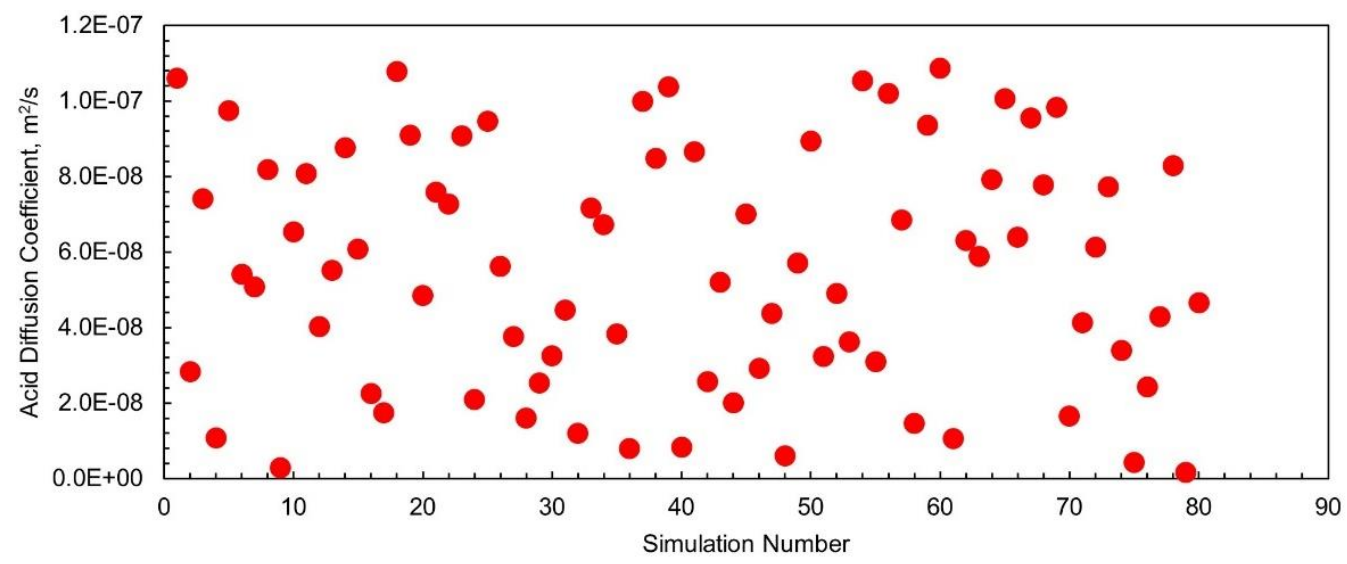

Fig. IV-6 Diffusion Coefficient used for the LHD ranged from 1.66E-9 to 1.09E-7 $\mathrm{m}^{2} / \mathrm{s}$. Reprinted with permission from (Kotb et al. 2021).

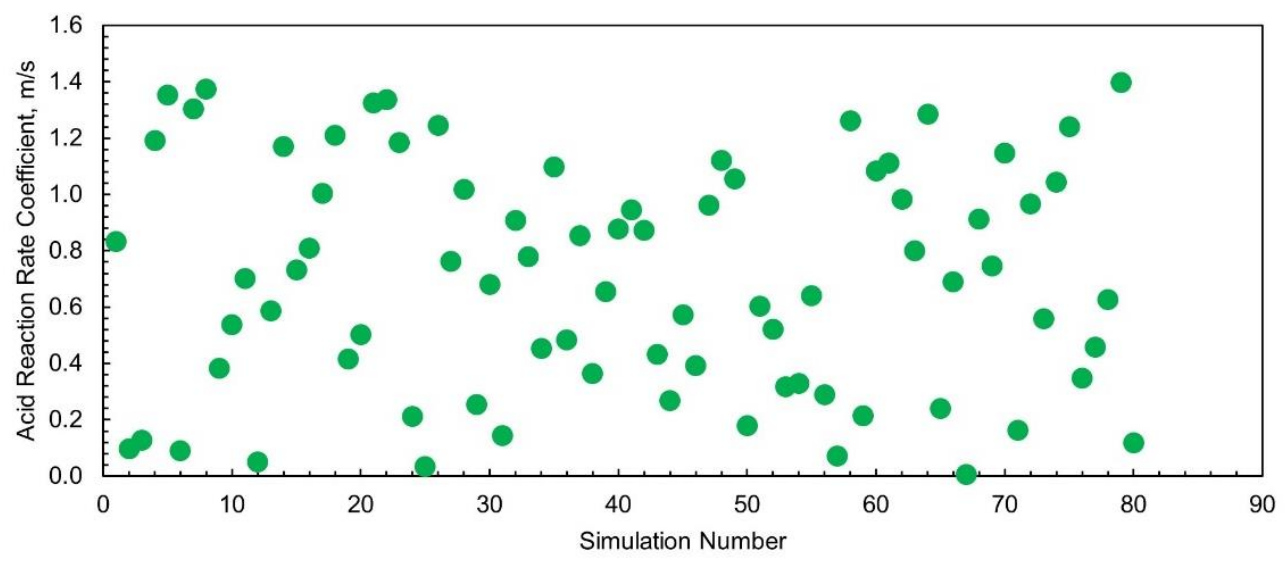

Fig. IV-7 Reaction rate coefficient used for the LHD ranged from $5.7 \mathrm{E}-3$ to $1.4 \mathrm{~m} / \mathrm{s}$. Reprinted with permission from (Kotb et al. 2021). 


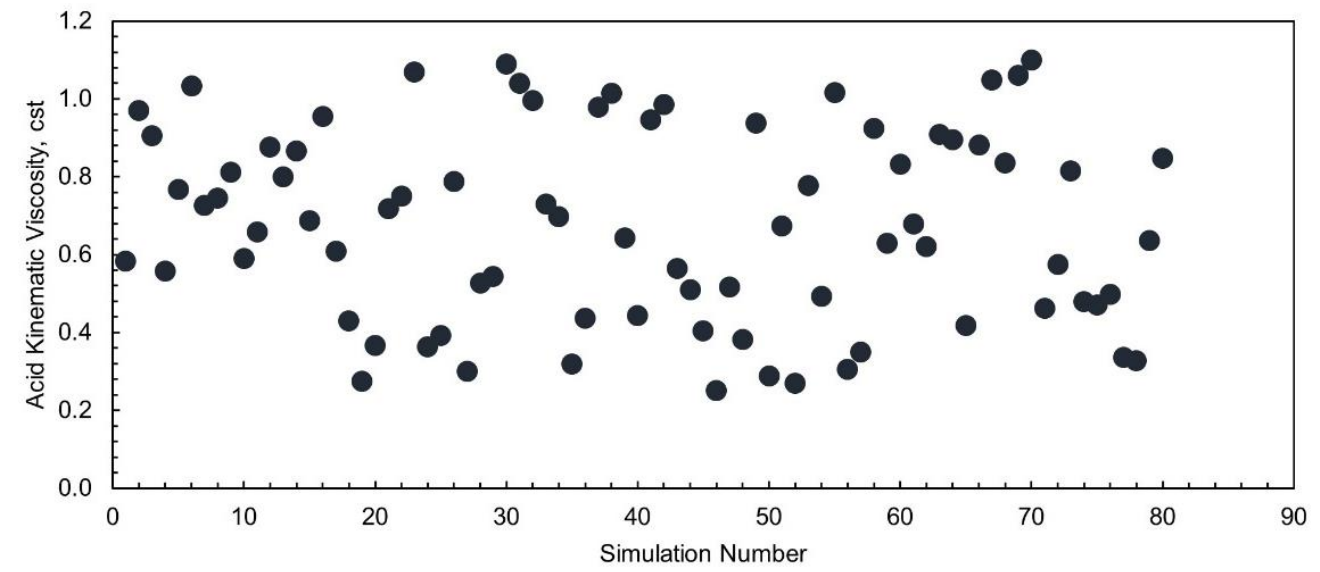

Fig. IV-8 Acid kinematic viscosity used for the LHD ranged from 0.25 to 1.1 cst. Reprinted with permission from (Kotb et al. 2021).

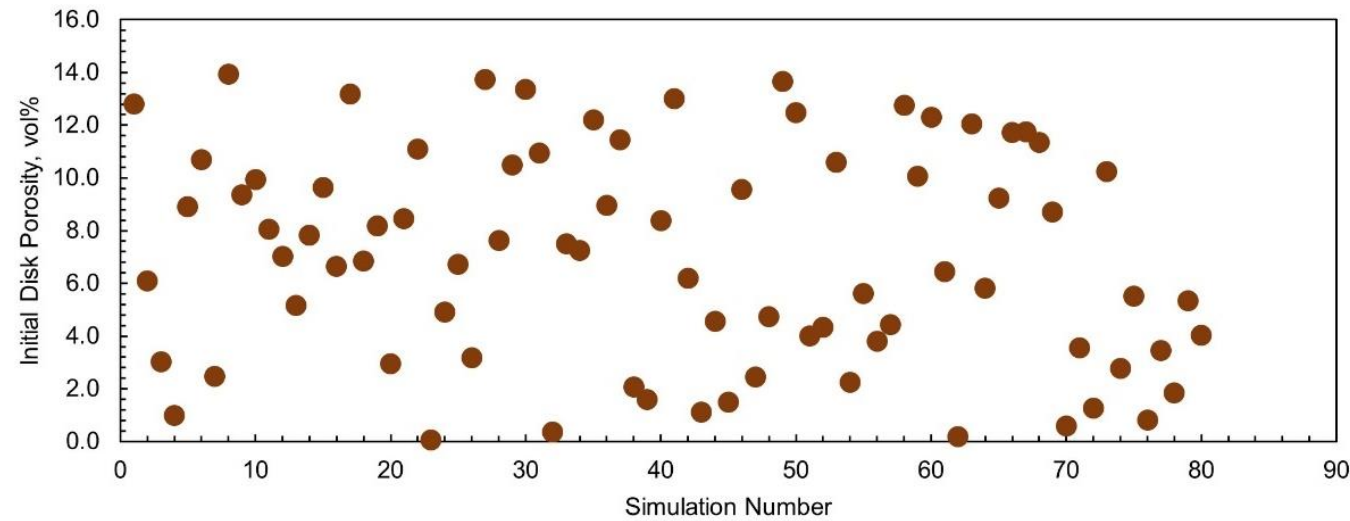

Fig. IV-9 Initial porosity of disk used for the LHD ranged from 0 to $14 \%$. Reprinted with permission from (Kotb et al. 2021).

The four inputs to the RDA experiment are known during physical experimentation. For simplicity, the 4-dimensional vector of input variables for the RDA experiment is denoted as $\boldsymbol{x}$, where $\boldsymbol{x}=\left[\phi, \omega, C_{b}, v\right]^{T}$. The CFD model takes as input the same input vector, $\boldsymbol{x}$, in addition to two more inputs: the diffusion coefficient and reaction rate coefficient, $D_{e}$ 
and $k$, respectively. While all variables in $x$ are physically adjustable in both the RDA and the CFD, and the coefficients $D_{e}$ and $k$ are not observed or measured in the RDA experiments but can be freely adjusted and controlled in the CFD model. The true values of these coefficients are unknown beforehand, and hence, have to be inferred using both the outputs from the RDA experiments and CFD model runs. This problem construction is known as statistical calibration, where the coefficients $D_{e}$ and $k$, being unobserved in the physical experiments yet freely manipulated in the CFD model, are referred to as the calibration parameters (Kennedy and O’hagan 2001).

In general, the essence of statistical calibration is to propose a linkage model that connects the outputs from the physical experiment with their computer-model counterparts to achieve the best match between them. In their work, Kennedy and O'hagan (2001) propose a linkage model to connect $y^{p}$ and $y^{c}$, which can be written using the following:

$y^{p}\left(\phi_{i}, C_{b i}, \omega_{i}, v_{i}\right)=y^{c}\left(\phi_{i}, C_{b i}, \omega_{i}, v_{i}, D_{e}, k\right)+\delta\left(\phi_{i}, C_{b i}, \omega_{i}, v_{i}\right)+e_{i} \quad i=$ $1, \ldots, n^{p}$

where $\delta\left(\phi_{i}, \omega_{i}, C_{b i}, v_{i}\right)$ is a bias correction term that accounts for any missing physics or simplifying approximations, which CFD models often entail. The exact form of this bias correction term will be discussed at the end of this section. The term $e_{i}$ is the independent and identically zero-mean normally distributed random variable representing the 
observational noise and $n^{p}$ is the total number of RDA experiments conducted. In this work, $n^{p}$ is 32 .

Since the CFD model in the current application is computationally expensive, a proxy model was developed to represent the response of the CFD model. The goal of the proxy model is to obtain a sufficiently accurate and computationally inexpensive prediction of $y^{c}$ at any arbitrary set of inputs. In this work, the Gaussian process (GP) model was used as a proxy model, which is a nonparametric statistical technique, widely used in the computer experiment literature as a proxy for physics-based computer simulators (Santner et al. 2003; Rasmussen and Williams, 2006; Ezzat et al. 2020). GP models can be written as follows:

$y^{c}\left(\phi_{i}, C_{b i}, \omega_{i}, v_{i}, D_{e i}, k_{i}\right)=\beta_{0}+\eta\left(\phi_{i}, C_{b i}, \omega_{i}, v_{i}, D_{e i}, k_{i}\right) \quad i=1, \ldots, n^{c}$,

where $\beta_{0}$ is the error term and $\eta($.$) is a zero-mean Gaussian process with its pairwise$ covariance defined as $\sigma_{i j}=\operatorname{Cov}\left(\eta_{i}, \eta_{j}\right)$. Note that for simplicity, this work uses the notation $\eta_{i}$ to denote $\eta\left(\phi_{i}, C_{b i}, \omega_{i}, v_{i}, D_{e i}, k_{i}\right)$. Let $\boldsymbol{\Sigma}$ be the $n^{c} \times n^{c}$ covariance matrix, for which the $(i, j)$ entry corresponds to $\sigma_{i j}$, where $n^{c}$ is the 80 simulations performed, then Eq. 2 can be rewritten as:

$\boldsymbol{Y}^{\boldsymbol{c}} \sim N\left(\beta_{0} \mathbf{1}_{n^{c} \times 1}, \boldsymbol{\Sigma}\right)$ 
where $\mathbf{1}_{n^{c} \times \mathbf{1}}$ is an $n^{c} \times 1$ vector of ones, and $\boldsymbol{Y}^{c}=\left[y_{1}^{c}, \ldots, y_{n^{c}}^{c}\right]^{T}$.

To fully characterize the GP model in Eq. 2 , the value of $\beta_{0}$ as well as the entries of the covariance matrix $\boldsymbol{\Sigma}$ have to be determined. For the former, the values of $\boldsymbol{Y}^{c}$ are standardized with a mean of 0 and standard deviation of 1 before fitting the GP, so $\beta_{0}$ can be set to 0 for the estimation procedure, and the values of $Y^{c}$ are scaled back to their original space once the estimation procedure is completed. Finding the entries of $\boldsymbol{\Sigma}$, however, is a more challenging task. A standard method in the GP modeling literature is to determine the entries of $\boldsymbol{\Sigma}$ through the specification of a positive-definite parametric covariance function, denoted by $K(.,$.$) . The automatic relevance determination squared$ exponential covariance function is a popular choice for $K(.$, ) (Rasmussen and Williams, 2006), which has a set of length-scale parameters in each dimension $\left\{\ell_{1}, \ldots, \ell_{6}\right\}$, as well as a sill parameter $\alpha$, and can be expressed as:

$K\left(\left(\phi_{i}, C_{b i}, \omega_{i}, v_{i}, D_{e i}, k_{i}\right),\left(\phi_{j}, C_{b j}, \omega_{j}, v_{j}, D_{e j}, k_{j}\right)\right)=\alpha \exp (-r)$

where

$$
\begin{aligned}
& r=\ell_{1}\left(\phi_{i}-\phi_{j}\right)^{2}+\ell_{2}\left(\omega_{i}-\omega_{j}\right)^{2}+\ell_{3}\left(C_{b i}-C_{b j}\right)^{2}+\ell_{4}\left(v_{i}-v_{j}\right)^{2}+\ell_{5}\left(D_{e i}-\right. \\
& \left.D_{e j}\right)^{2}+\ell_{6}\left(k_{i}-k_{j}\right)^{2}
\end{aligned}
$$


The parameters in Eq. 4 can be estimated using the maximum likelihood estimation (MLE) approach. Given the parameter estimates, a closed-form expression for the point prediction of the proxy model at any arbitrary design point $\left[\phi^{*}, \omega^{*}, C_{b}{ }^{*}, v^{*}, D_{e}^{*}, k^{*}\right]^{T}$ can be obtained as:

$\hat{y}^{c}\left(\phi^{*}, \omega^{*}, C_{b}{ }^{*}, v^{*}, D_{e}^{*}, k^{*}\right)=\boldsymbol{\sigma}^{T} \boldsymbol{\Sigma}^{-1} \boldsymbol{Y}^{c}$

where $\boldsymbol{\sigma}$ is the $n^{c} \times 1$ vector of covariances between $\boldsymbol{Y}^{c}$ and $\hat{y}^{c}\left(\phi^{*}, \omega^{*}, C_{b}{ }^{*}, v^{*}, D_{e}^{*}, k^{*}\right)$. With the proxy model in place, $y^{c}$ in Eq. 2 can be replaced by its proxy model prediction $\hat{y}^{c}$ in Eq. 7, and the model in Eq. 1 can be re-written as:

$$
\begin{aligned}
& y^{p}\left(\phi_{i}, C_{i}, \omega_{i}, v_{i}\right)=\hat{y}^{c}\left(\phi_{i}, C_{i}, \omega_{i}, v_{i}, D_{e}, k\right)+\delta\left(\phi_{i}, C_{i}, \omega_{i}, v_{i}\right)+e_{i}, \quad i= \\
& 1, \ldots, n^{p}
\end{aligned}
$$

To estimate the linkage model in Eq. 7, Kennedy and O'Hagan (2001) proposed a hierarchical framework to perform two tasks simultaneously: (i) correct the bias of the computer model by estimating the function $\delta($.$) And (ii) find the estimates for the$ calibration parameters, namely: $D_{e}$ and $k$, that achieve the best agreement between $y^{p}$ and $y^{c}$. While their approach has shown to be widely useful for a set of applications, a limitation of their approach is the implicit assumption that the calibration parameters $D_{e}$ and $k$ have constant values across the domain of the observable input variables in $\boldsymbol{x}$. 
However, $D_{e}$ and $k$ are not constants, and are functions of some of the observable inputs in $\boldsymbol{x}$, like $C_{b}$ and $v$. In light of that, Eq. 7 will be used in a way where instead of finding constant values for $D_{e}$ and $k$ as traditionally implemented in Kennedy and O'hagan (2001), the functional relationships $D_{e}\left(C_{b}, v\right)$ and $k\left(C_{b}, v\right)$ that realize the best agreement between $y^{p}$ and $y^{c}$ would be estimated.

Multiple applications have been recently reported in the literature where the calibration parameters are not fixed over the domain of the observable input variables (Bayarri et al. 2007; Pourhabib et al. 2015; Plumlee et al. 2016; Brown and Atamturktur 2018). The formulation proposed by Pourhabib et al. (2015) and Ezzat et al. (2018) is followed to estimate the functions $D_{e}($.$) and k($.$) , by minimizing a penalized distance between y^{p}$ and $\hat{y}^{c}$ as:

$$
\begin{aligned}
& \min _{D e, C} \frac{1}{n^{p}} \sum_{i=1}^{n^{p}}\left\{y^{p}\left(\phi_{i}, C_{b i}, \omega_{i}, v_{i}\right)-\hat{y}^{c}\left(\phi_{i}, C_{b i}, \omega_{i}, v_{i}, D_{e}\left(C_{b i}, v_{i}\right), k\left(C_{b i}, v_{i}\right)\right)-\right. \\
& \left.\delta\left(\phi_{i}, C_{i}, \omega_{i}, v_{i}\right)\right\}^{2}+\lambda_{1}\|D e\|_{\aleph_{\vartheta_{1}}}+\lambda_{2}\|k\|_{\aleph_{\vartheta_{2}}}
\end{aligned}
$$

where $\lambda_{1}$ and $\lambda_{2}$ are smoothing parameters, $D_{e}$ and $k$ lie in the native spaces $\aleph_{\vartheta_{1}}$ and $\aleph_{\vartheta 2}$, generated by the kernel functions $\vartheta_{1}$ and $\vartheta_{2}$, respectively. For both $\vartheta_{1}$ and $\vartheta_{2}$, a squared exponential kernel is used, similar to the one in Eq. 3, for which the parameters can be estimated simultaneously while solving Eq. 8. 


\section{Results and Discussion}

\section{Experimental Results}

Table IV-1 summarizes the results from the RDA experiments, and Fig. IV-10 and Fig. IV-11 show the disks after the RDA experiments. After the experiments were performed, the diffusion coefficient was calculated using the ICP data and the properties of the acid. Fig. IV-12 and Fig. IV-13 summarize the rate of $\mathrm{H}^{+}$transfer to the disk obtained from the ICP measurements. Table IV-2 summarizes the diffusion coefficient calculated from the different experiments. Disk rotational speed, acid concentration, and rock type all have an impact on the dissolved rock fraction measured and the diffusion coefficient calculated. From Table B-1, it can be observed that the amount of rock dissolved increases as the rotational speed increases. At $250^{\circ} \mathrm{F}$, the amount of rock dissolved increased with increasing molecular weight of acid from 0.25 to $4.83 \mathrm{M}$ since the reaction is dominated by mass transfer. Finally, the amount of rock dissolved in Indiana limestone was higher than in the case of marble. This results from higher acid concentration and higher porosity in the limestone which increases the area exposed to the acid over marble disks. The diffusion coefficient increased with temperature. The diffusion coefficient also decreased with molarity. Similar behavior was observed by Lobo et al. (1979) and Rabie et al. (2014). The only exception was that the calculated value of $D_{e}$ increased from 1.25 to $4.83 \mathrm{M}$ at $250^{\circ} \mathrm{F}$. This increase can be a result of the assumptions in the equation not being met; in particular, the assumption that the area of the surface of the disk remains constant throughout the experiment. The increase of $D_{e}$ at $4.83 \mathrm{M}$ from $1.25 \mathrm{M}$ can be explained by the increase in surface area of the disk, which is not considered in the equation developed 
by Newman (1966). Another reason for this discrepancy can be a result of the inherent heterogeneity of the limestone rock that is also not considered. All RDA experiments assume homogenous rock. However, marble disks and limestone disks can react differently with acid because of heterogeneity, even though they both consist of calcite.

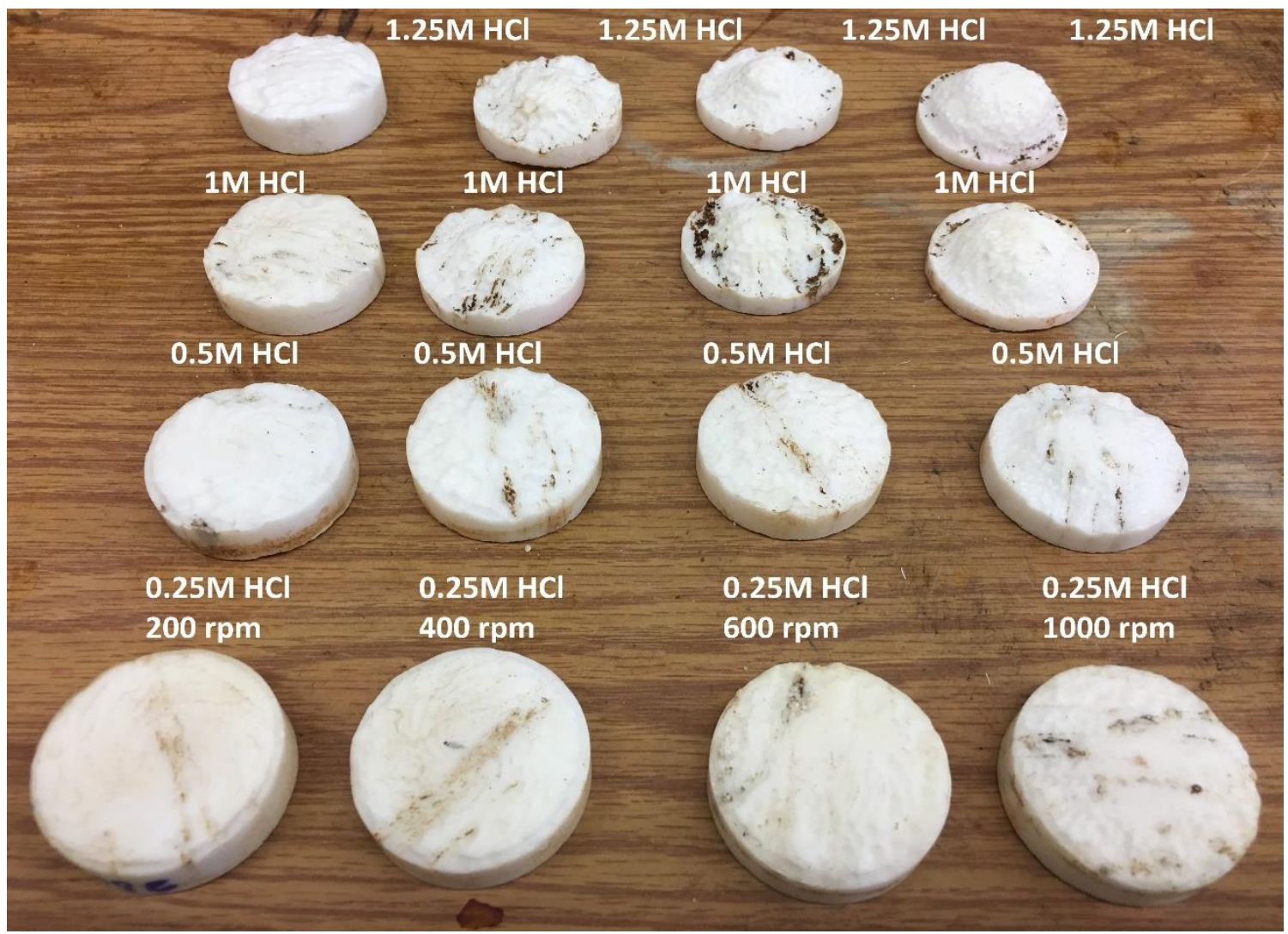

Fig. IV-10 Marble disks after the RDA experiments. Reprinted with permission from (Kotb et al. 2021). 


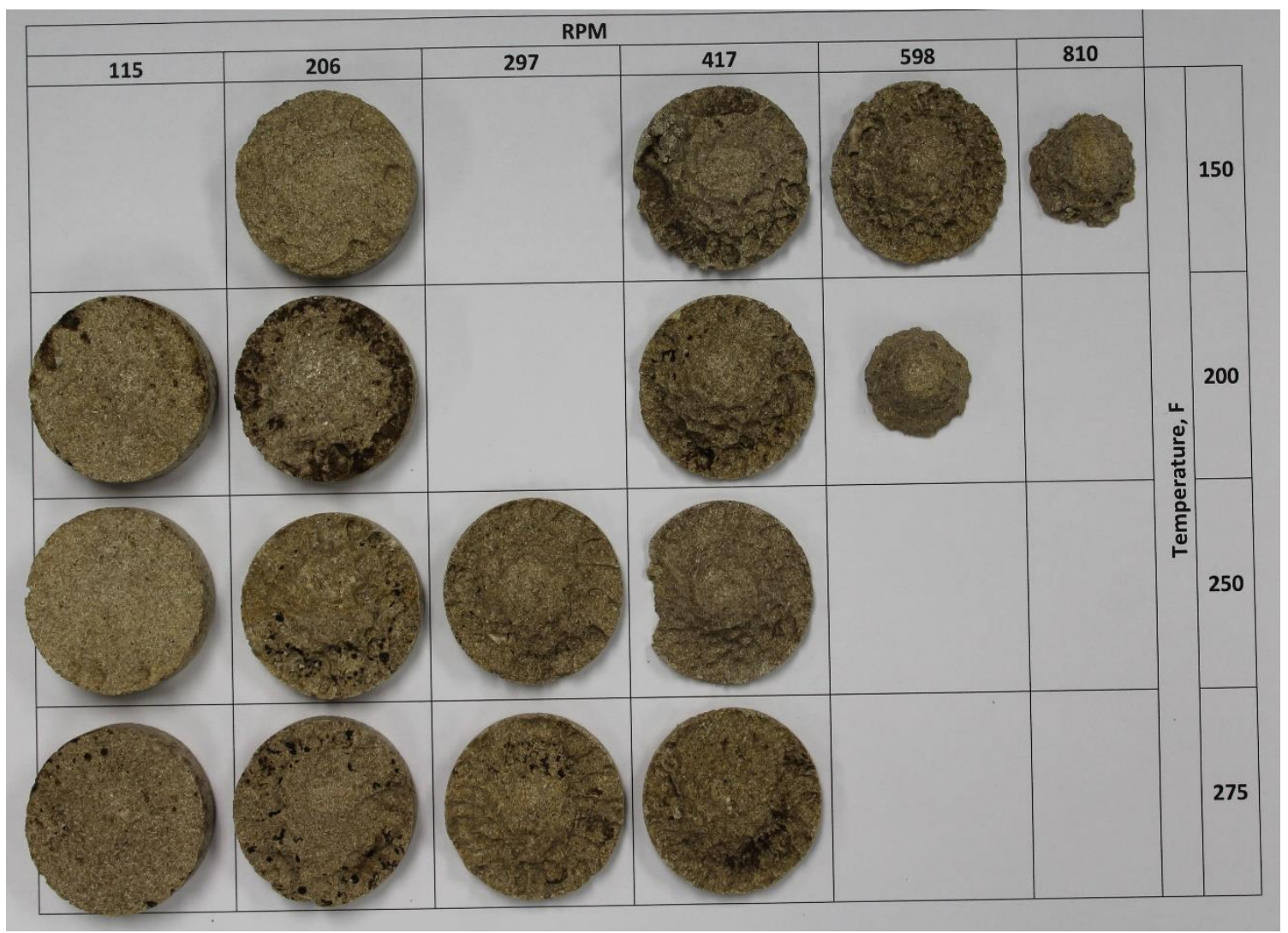

Fig. IV-11 Indiana limestone disks after the RDA experiments. Reprinted with permission from (Kotb et al. 2021). 


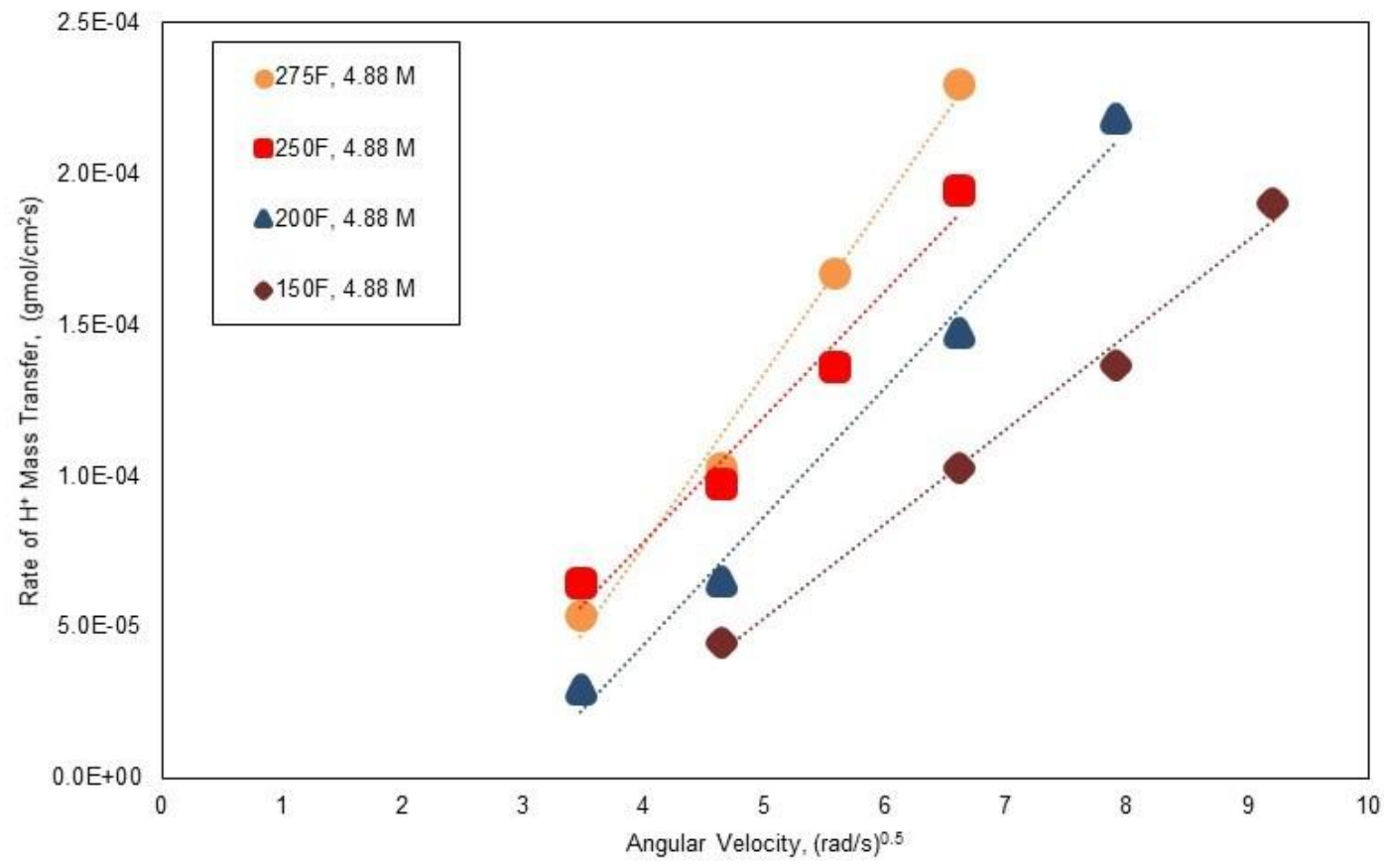

Fig. IV-12 Rate of $\mathrm{H}^{+}$mass transfer to the disk obtained from the ICP data showing the impact of temperature at $4.83 \mathrm{M}$ HCl. Reprinted with permission from (Kotb et al. 2021). 


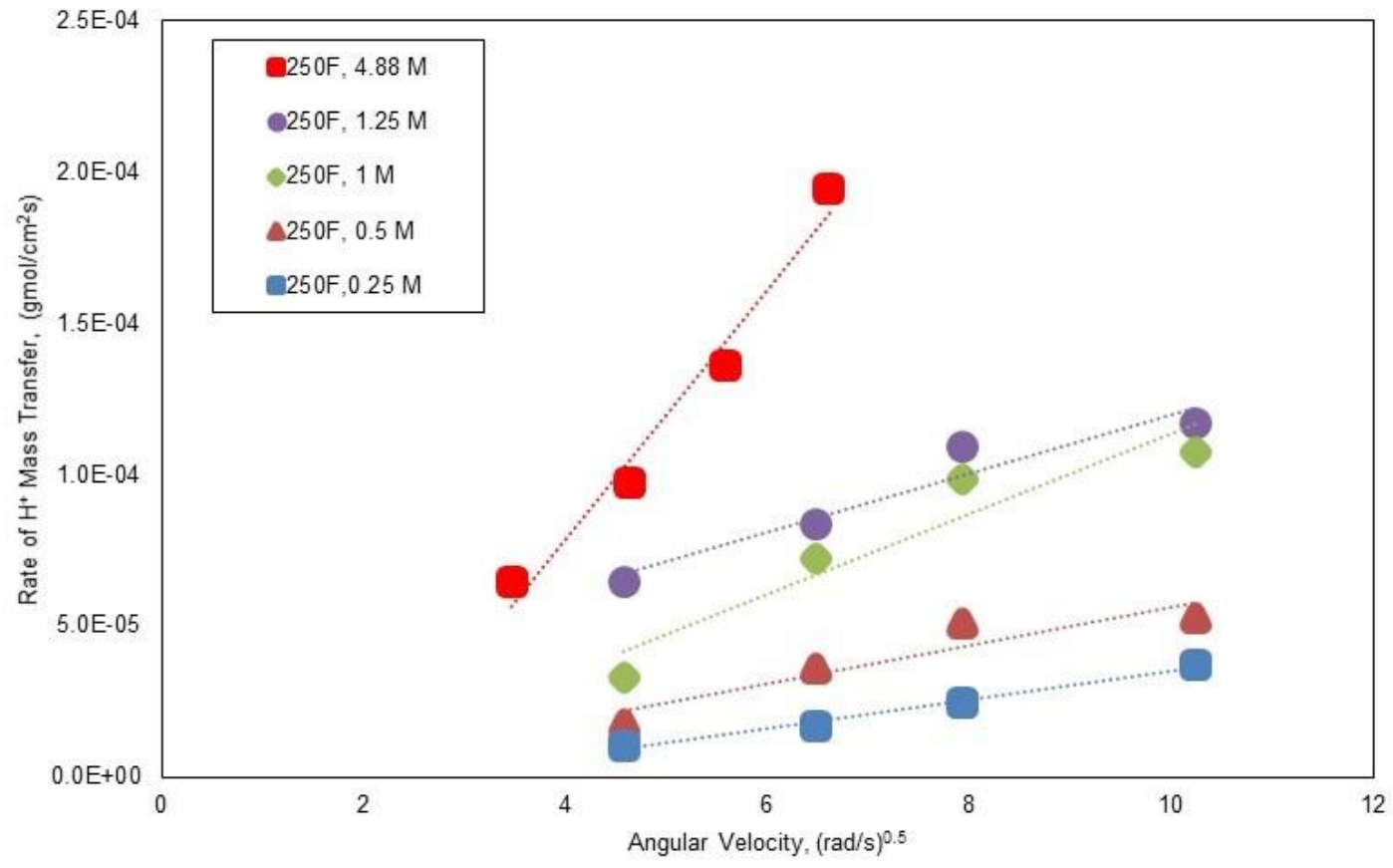

Fig. IV-13 Rate of $\mathrm{H}^{+}$mass transfer to the disk obtained from the ICP data showing the impact of $\mathrm{HCl}$ concentration at $250^{\circ} \mathrm{F}$. Reprinted with permission from (Kotb et al. 2021).

\begin{tabular}{ccccc}
$\begin{array}{c}\text { Temperature } \\
(\text { F })\end{array}$ & $\begin{array}{c}\text { Concentration } \\
(\mathrm{M})\end{array}$ & $\begin{array}{c}\text { Diffusion Coefficient } \\
\text { Calculated Using } \\
\text { Newman }(1966)\left(\mathrm{m}^{2} / \mathrm{s}\right)\end{array}$ & $\begin{array}{c}\text { Diffusion } \\
\text { Coefficient } \\
\text { Estimated } \\
\text { from the } \\
\text { Model } \\
\left(\mathrm{m}^{2} / \mathrm{s}\right)\end{array}$ & $\begin{array}{c}\text { Reaction } \\
\text { Rate } \\
\text { Coefficient } \\
\text { Estimated } \\
\text { from the } \\
\text { Model }(\mathrm{m} / \mathrm{s})\end{array}$ \\
\hline 150 & 4.83 & $3.99 \mathrm{E}-08$ & $3.58 \mathrm{E}-08$ & $4.52 \mathrm{E}-9$ \\
200 & 4.83 & $6.16 \mathrm{E}-08$ & $4.96 \mathrm{E}-08$ & 0.008 \\
250 & 4.83 & $7.57 \mathrm{E}-08$ & $5.02 \mathrm{E}-08$ & 0.013 \\
275 & 4.83 & $9.24 \mathrm{E}-08$ & $4.87 \mathrm{E}-08$ & 0.014 \\
250 & 1.25 & $3.90 \mathrm{E}-08$ & $2.64 \mathrm{E}-08$ & 0.017 \\
250 & 1.00 & $9.80 \mathrm{E}-08$ & $2.43 \mathrm{E}-08$ & 0.017 \\
250 & 0.50 & $1.00 \mathrm{E}-07$ & $2.05 \mathrm{E}-08$ & 0.016 \\
250 & 0.25 & $2.15 \mathrm{E}-07$ & $1.87 \mathrm{E}-08$ & 0.016 \\
\hline
\end{tabular}

Table IV-2 Calculated diffusion coefficient using Newman's (1966) equation. The estimated diffusion coefficient and rection rate coefficient estimated from the model. Reprinted with permission from (Kotb et al. 2021). 


\section{Simulation Results}

First, the bias term $\delta($.$) in Eq. 7$ needs to be defined. $\delta($.$) can be modeled through any$ choice of parametric or nonparametric functions. For instance, $\delta($.$) can be modeled using$ another GP model. Instead, the preliminary analysis of the data and the physical understanding of the process are used to guide the selection of the bias term. Fig. IV-14 shows the boxplots of the outputs from the RDA experiment and the CFD model. It is apparent that, in general, the CFD model overestimates the dissolved rock. This results from the assumption by Newman (1966) that the area of the disk does not change during the reaction, which can result in an overestimation of the $D_{e}$ value. This assumption results in an overestimation of the dissolved rock when the estimated $D_{e}$ value from the RDA experiment is used in the CFD. 


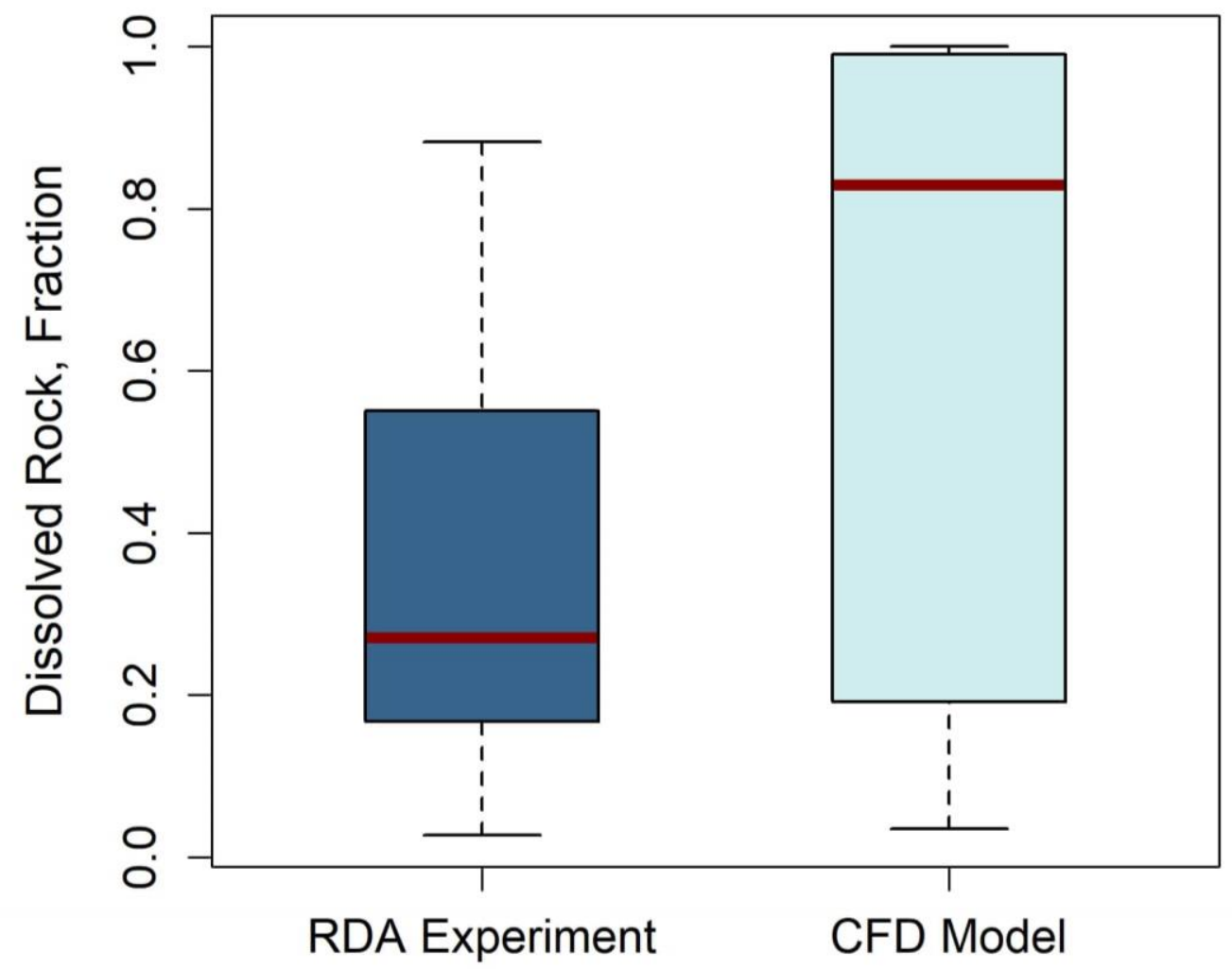

Fig. IV-14 Bias between the RDA experiments and CFD model runs. Reprinted with permission from (Kotb et al. 2021).

Moreover, the observational analysis suggests that the bias of the CFD model varies with concentration and disk rotational speed. Specifically, as evident by Fig. IV-15 and Fig. IV-16, the CFD model appears to overestimate the dissolved rock at higher concentrations. This overestimation can be explained by the large area exposed to acid at high concentrations and rotational speeds due to the high dissolution rate. This exposed disk area to acid amplifies the error associated with the assumption that the area of the disk remains constant throughout the reaction. 


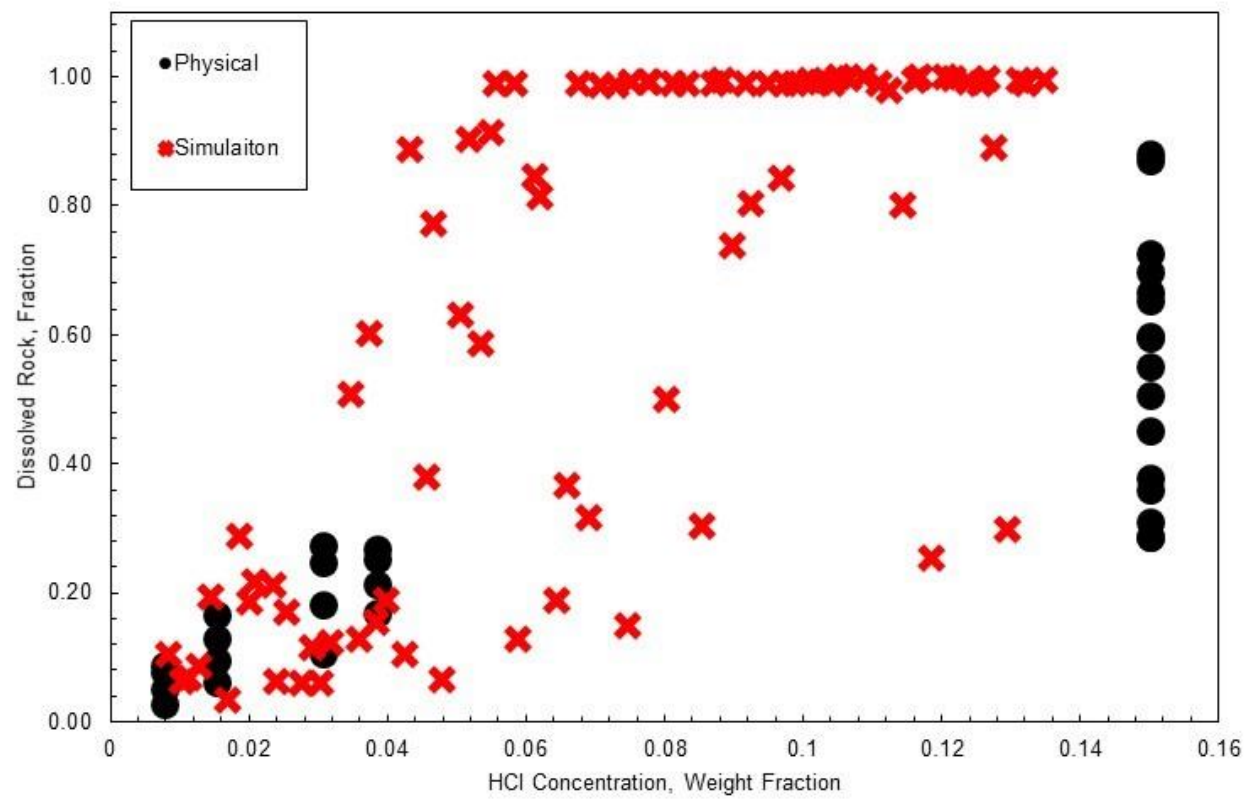

Fig. IV-15 Outputs from the RDA experiments versus CFD model runs as a function of concentration. Reprinted with permission from (Kotb et al. 2021).

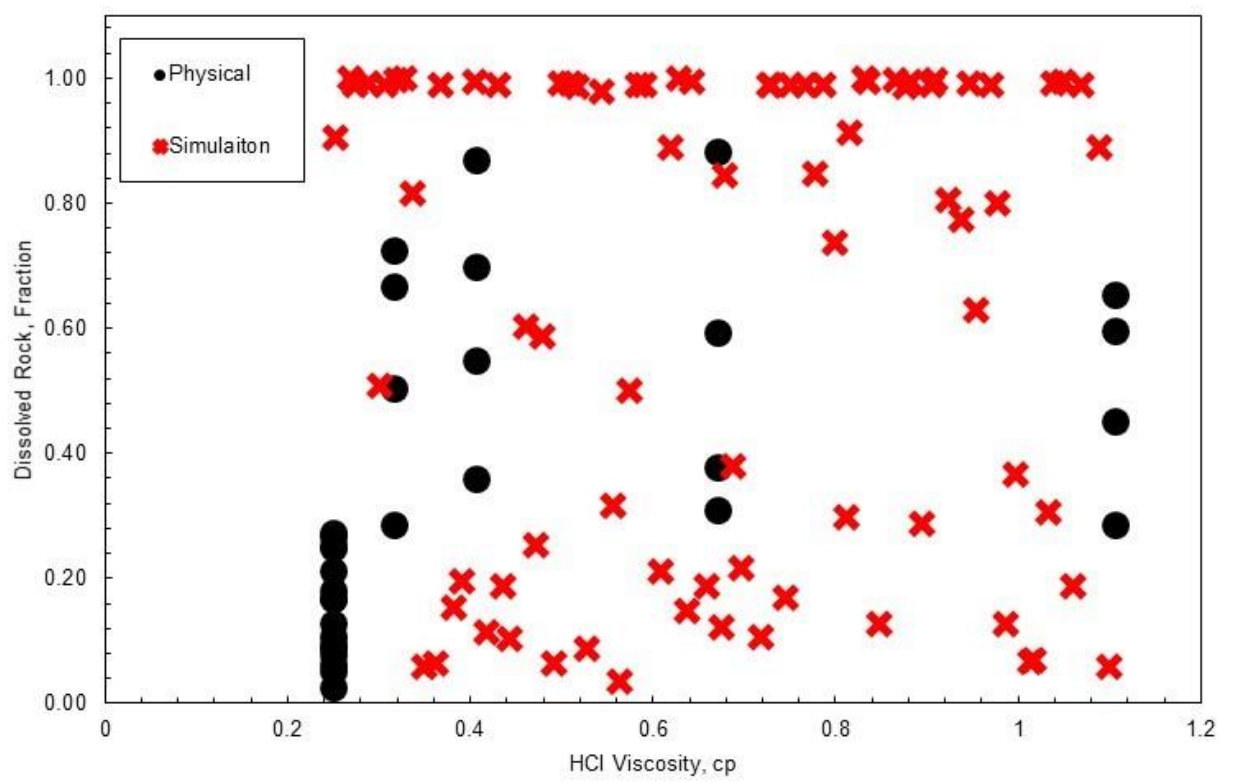

Fig. IV-16 Outputs from the RDA experiments versus CFD model runs as a function of acid viscosity. Reprinted with permission from (Kotb et al. 2021). 
As such, $\delta($.$) was modeled as a function of both C_{b}$ and $\omega$. The bias term was modeled as a linear function of both acid concentration and disk rotational speed, as follows:

$\delta=b_{0}+b_{1} C+b_{2} \omega$

where $b_{0}, b_{1}$, and $b_{2}$ are constants.

With all the components in Eq. 8 defined, the optimization problem is solved using a traditional gradient-descent-based method. The outputs of the optimization problem are the estimated functional relationships $D_{e}($.$) and k($.$) , as well as the bias correction$ parameters. Once the estimation is completed, the prediction of the dissolved rock can be obtained at any arbitrary set of input values $\left[\phi^{*}, \omega^{*}, C_{b}^{*}, v^{*}\right]^{T}$ as:

$\hat{y}\left(\phi^{*}, \omega^{*}, C_{b}^{*}, v^{*}\right)=\hat{y}^{c}\left(\phi^{*}, \omega^{*}, C_{b}^{*}, v^{*}, \widehat{D e}\left(C_{b}^{*}, v^{*}\right), \hat{k}\left(C_{b}^{*}, v^{*}\right)\right)+\hat{\delta}\left(\omega^{*}, C_{b}^{*}\right)$,

where $\widehat{D_{e}}$ and $\widehat{k}$ are the estimated values from the functional estimation and $\hat{\delta}\left(\omega^{*}, C_{b}^{*}\right)$ is the bias term obtained from Eq. 9.

To demonstrate the predictive ability of the final calibrated model, six points out of the 32 physical points used to validate the model were randomly selected and reserved, and the 
remaining 26 data points were used to construct the model. To further showcase the robustness of this approach, a cross-validation was performed by repeating the process mentioned earlier 100 times, where, at each time, six different points were reserved for testing. For demonstration purposes, the results from one of the arbitrary samples are shown, and later, the aggregate prediction performance is shown from the 100 randomly selected samples.

First, as mentioned above, the GP model was fitted to the 80 data points obtained from the CFD model. One of the main advantages of the GP model is its interpolative property, meaning the constructed response surface passes through the training data points. This property is highly desired for deterministic computer models, like the CFD model presented in this work. Fig. IV-17 and Fig. IV-18 show the point predictions of the proxy model obtained at the 80 training data points. The point predictions were obtained using Eq. 6 and successfully interpolated the training points. This proxy model is 6-dimensional, however, the response as a function of acid concentration and viscosity are only shown for demonstration purposes. 


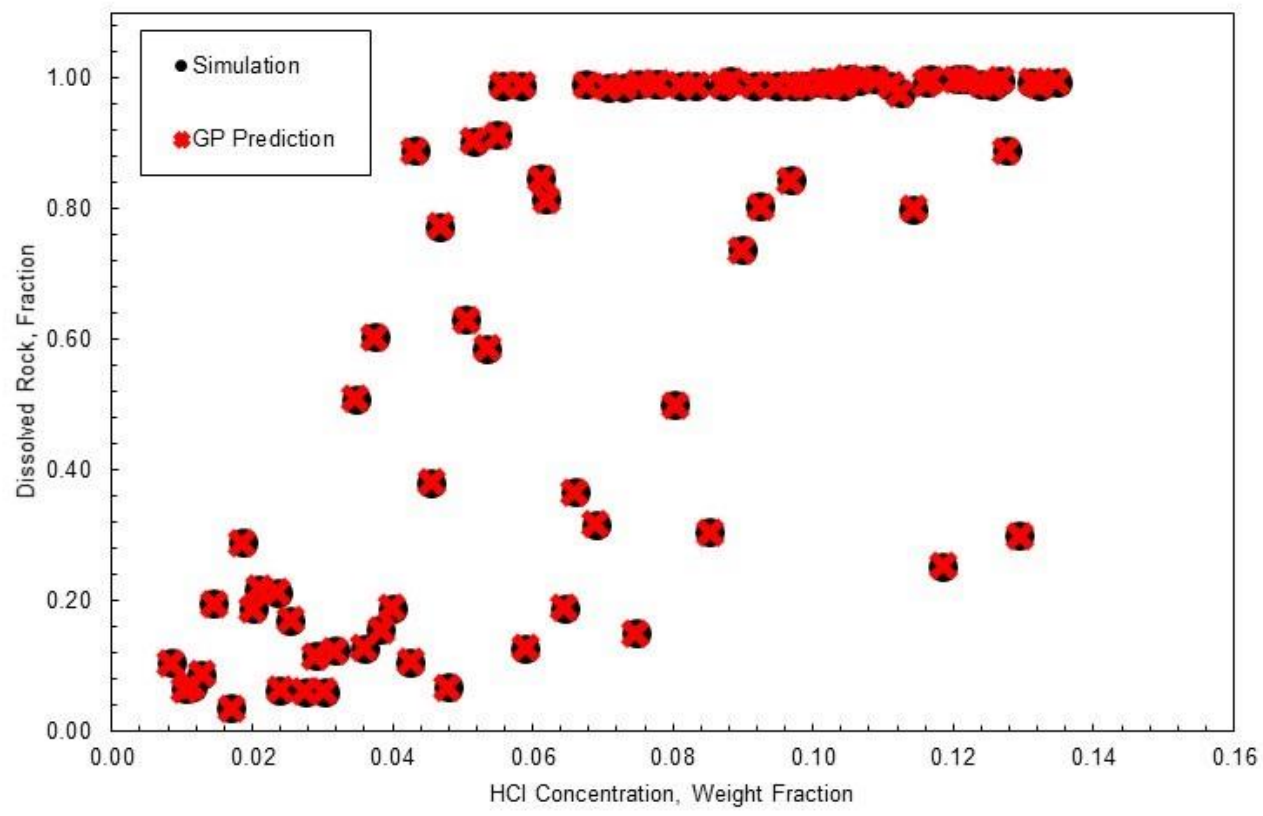

Fig. IV-17 The predictions from the proxy model at the 80 design points as a function of concentration. Reprinted with permission from (Kotb et al. 2021).

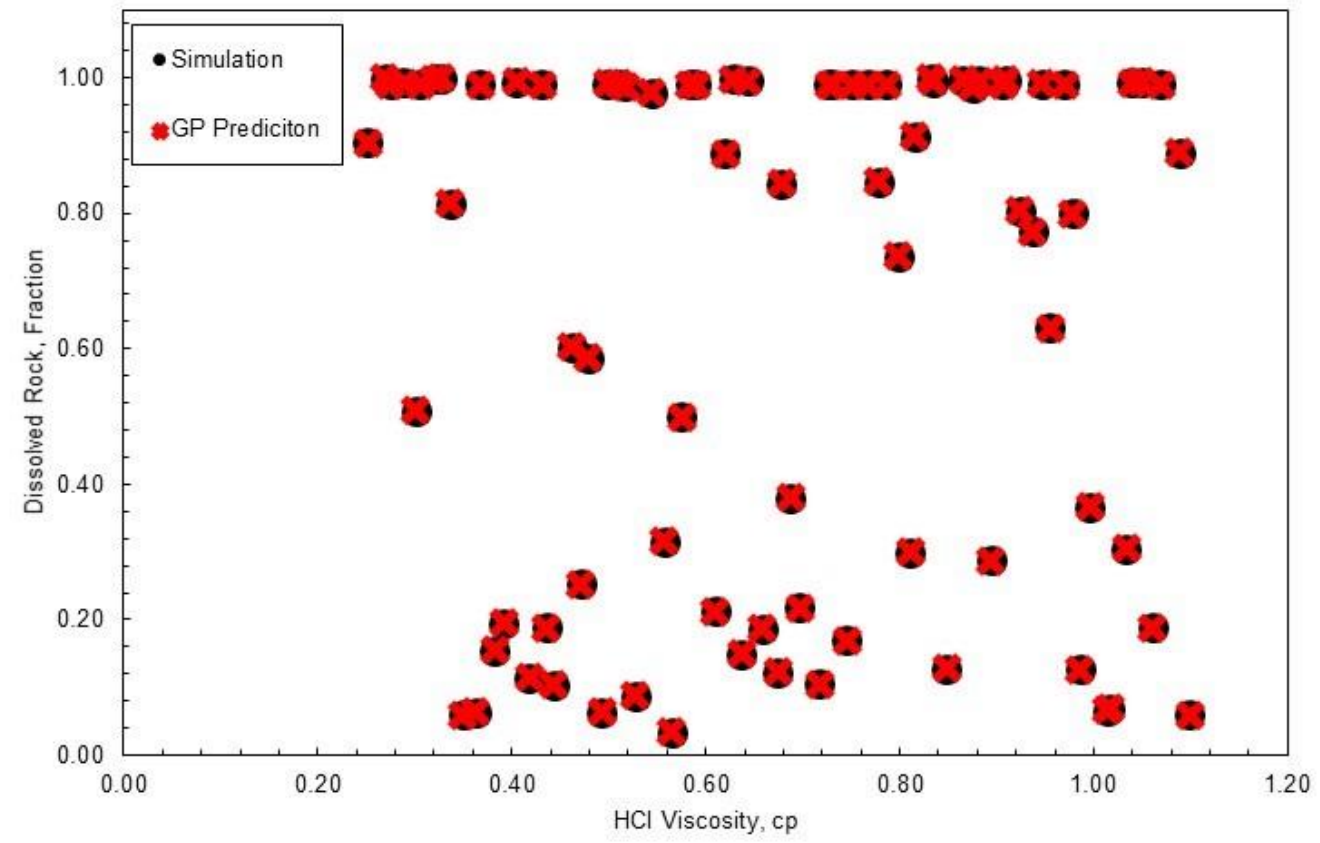

Fig. IV-18 The predictions from the proxy model at the 80 design points as a function of acid viscosity. Reprinted with permission from (Kotb et al. 2021). 
Next, the optimization problem in Eq. 7 was numerically solved using the routine nonlinear minimization in the statistical programming language R. In this implementation, it was found that $\lambda_{1}=\lambda_{2}=0.01$. By carefully selecting a suitable starting point and step size for the numerical optimization, physically feasible values for $D_{e}$ and $k$ were ensured. Table IV-2 shows the estimated values of $D_{e}$ and $k$ at the different test conditions. Table IV-3 shows the model's predictive capability by comparing the physical experiment results with the predicted dissolved rock using the developed model and Newman's method. From the table, The proposed model performs better than Newman's calculation. However, some discrepancies can be observed between the predictions of the developed model and the experimental results. These discrepancies can be explained by the bias term's (Eq. 9) linear function choice, which lacks some of the underlying physics. This is especially apparent in the $1 \mathrm{M}$ marble case at $200 \mathrm{rpm}$ where the bias term results in underestimating the amount of rock dissolved. 


\begin{tabular}{|c|c|c|c|c|c|c|c|}
\hline $\begin{array}{l}\text { Rock } \\
\text { Type }\end{array}$ & $\begin{array}{c}\text { Temperat } \\
\text { ure }(\mathrm{F})\end{array}$ & $\begin{array}{c}\text { Initial } \\
\text { Porosity } \\
\text { (wt } \%)\end{array}$ & $\begin{array}{l}\mathrm{HCl} \\
\text { Concent } \\
\text { ration } \\
(\mathrm{M})\end{array}$ & $\begin{array}{l}\text { Rotation } \\
\text { al Speed } \\
(\mathrm{rpm})\end{array}$ & $\begin{array}{l}\text { Newman } \\
\text { 's } \\
\text { Predictio } \\
\text { n of } \\
\text { Rock } \\
\text { Dissolve } \\
\text { d (wt } \%) \\
\end{array}$ & $\begin{array}{l}\text { Proposed } \\
\text { Model } \\
\text { Prediction } \\
\text { of Rock } \\
\text { Dissolved } \\
\quad(w t \%)\end{array}$ & $\begin{array}{c}\text { Actual } \\
\text { Rock } \\
\text { Dissolved } \\
\text { (wt } \%)\end{array}$ \\
\hline \multirow{2}{*}{$\begin{array}{c}\text { Indiana } \\
\text { Limestone }\end{array}$} & 200 & 12.5 & 4.83 & 100 & 23 & 23 & 28 \\
\hline & 275 & 12.3 & 4.83 & 100 & 42 & 31 & 32 \\
\hline \multirow{4}{*}{ Marble } & 250 & 0 & 0.25 & 400 & 63 & 10 & 12 \\
\hline & 250 & 0 & 0.50 & 1000 & 58 & 25 & 28 \\
\hline & 250 & 0 & 1.00 & 200 & 7 & 10 & 24 \\
\hline & 250 & 0 & 1.25 & 400 & 43 & 34 & 38 \\
\hline
\end{tabular}

Table IV-3 Comparison between the dissolved rock obtained using Newman's (1966) equation, the proposed model and the actual experimental value. Reprinted with permission from (Kotb et al. 2021).

Using the estimated functions $D_{e}($.$) and k($.$) , and the estimated bias term parameters, point$ predictions for the reserved test points can be obtained by plugging them into Eq. 10. The prediction quality was evaluated using the mean square error (MSE) and the percentage of accuracy-precision (PAP) (Heidaryan 2019), defined in Eqs. 11 and 12, respectively:

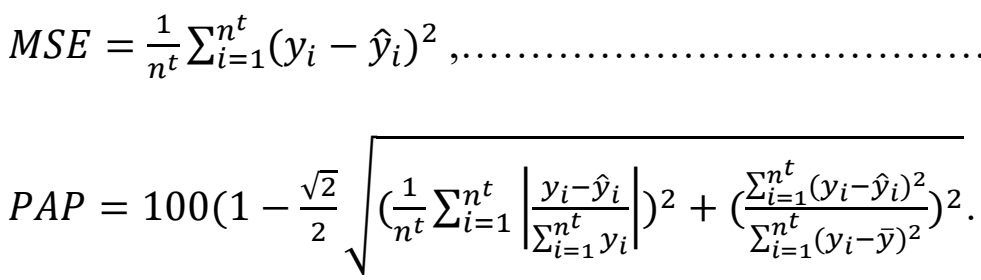

where $y_{i}$ and $\hat{y}_{i}$ are the actual observation and the final model prediction of the dissolved rock, respectively, $\bar{y}$ is the average of the actual observations, and $n^{t}$ is the number of points reserved for testing. In this case, $n^{t}$ is 6 . In the PAP method, the denominator in the 
absolute percent error was set to $\sum_{i=1}^{n^{t}} y_{i}$ to offset the small scale of rock dissolved and ensure numerical stability. The dissolved rock was compared using the $D_{e}$ obtained from this model and the calculated $D_{e}$ value obtained from Newman's (1966) equation. For this model, an MSE of 0.032 was obtained on average, while using Newman's (1966) equation, the MSE was 0.068 on average. Moreover, the PAP showed $62 \%$ on average for the developed model compared to $38 \%$ using Newman's method.

The boxplots of the MSE and PAP from the 100 samples are shown in Fig. IV-19 and Fig. IV-20, respectively. The figures suggest that, on average, the proposed method can achieve an improvement of 53\% and $63 \%$ in terms of MSE and PAP, respectively, relative to Newman's (1966) method in estimating the dissolved disk using $D_{e}$ and $k$. It is also worth mentioning that in the latter approach, although an empirical estimate for $D_{e}$ is used, the functional estimate of $k$ obtained from this model is also used. Thus, further improvements from the presented approach can be expected since the $k$ value is unknown.

This work introduced the first model that calculates the reaction rate coefficient in the $\mathrm{HCl}$ limestone reaction. This model saves time and experimental cost in obtaining the reaction kinetics from the RDA. First, it only requires one experiment to obtain the reaction kinetics instead of the standard three to four experiments typically performed. Second, it uses the amount of rock dissolved for its calculation instead of the amount of calcium in the RDA experiments' effluent. This prevents the dependency on effluent chemical analysis using the ICP, which is expensive and not readily available. Finally, this model has improved the prediction of the amount of rock dissolved by up to $63 \%$ over 
Newman's traditional method. The accurate determination of the reaction kinetic values is crucial in optimizing the amount of acid and injection rate needed in an acid treatment job.

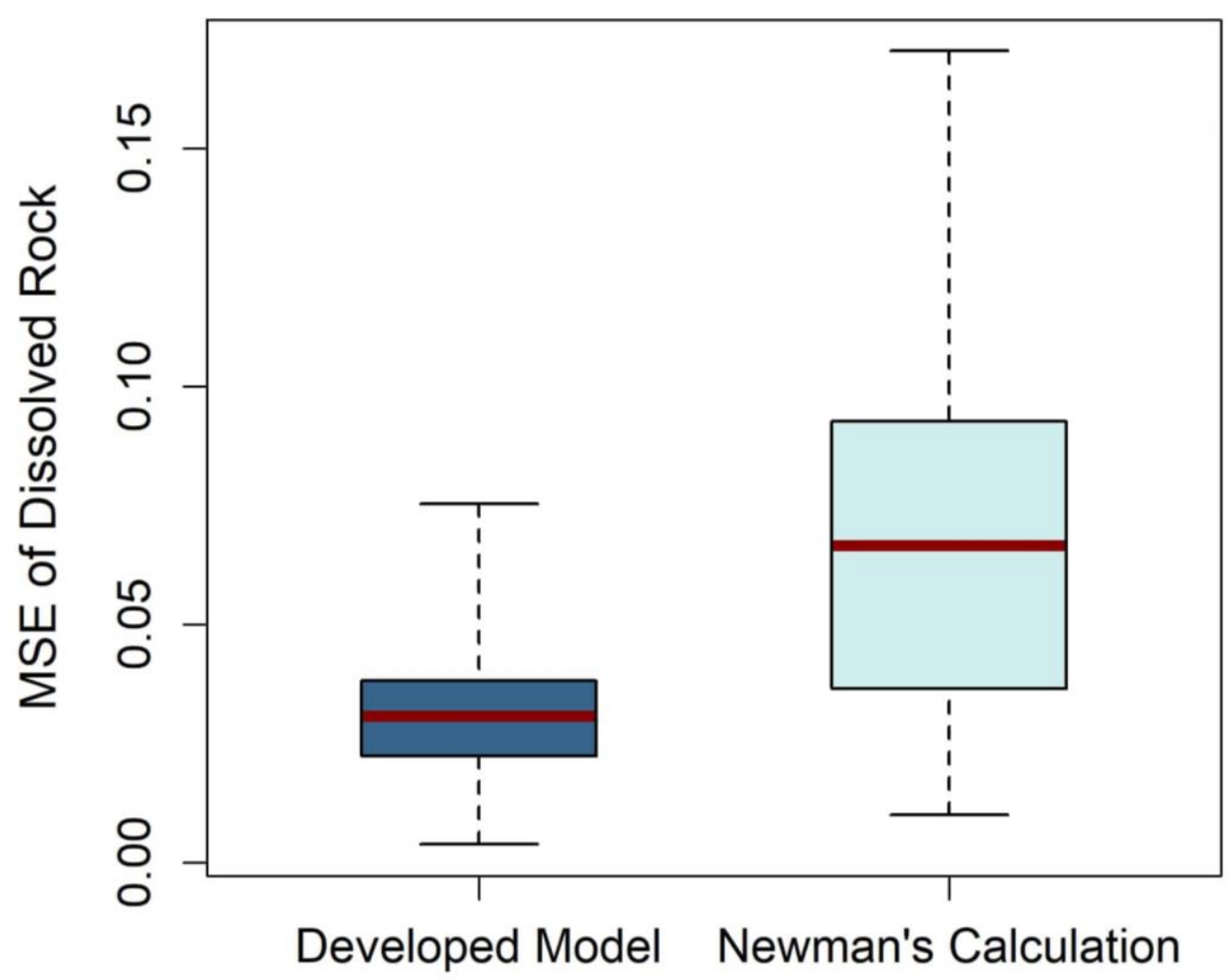

Fig. IV-19 Boxplots of the MSE from 100 samples using the developed model (on the left) versus Newman's calculation (1966) (on the right). Reprinted with permission from (Kotb et al. 2021). 


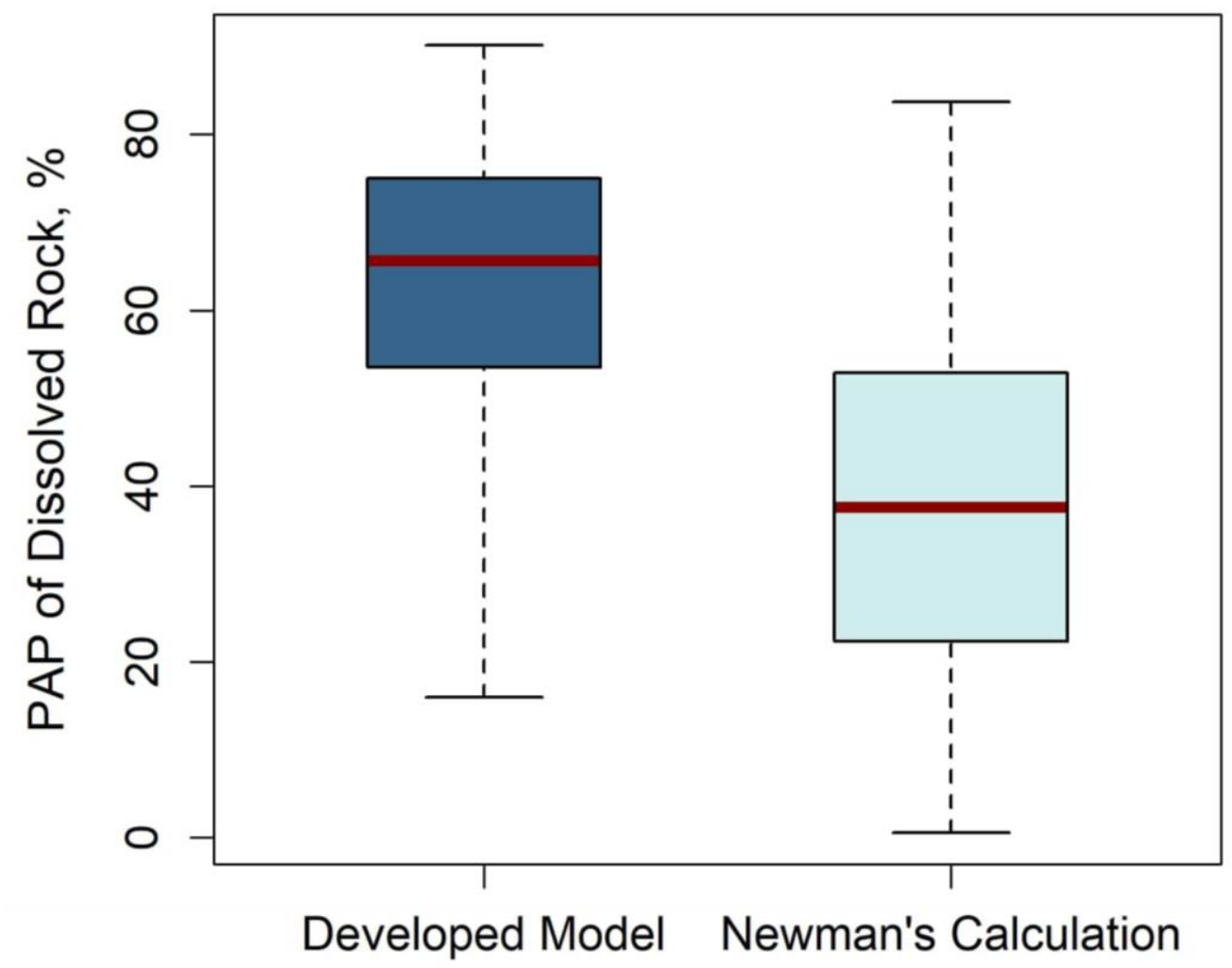

Fig. IV-20 Boxplots comparison of the PAP of dissolved rock from 100 samples using the developed model (on the left) versus Newman's calculation (1966) (on the right). Reprinted with permission from (Kotb et al. 2021). 


\section{CHAPTER V}

\section{CONCLUSIONS, RECOMMENDATIONS AND FUTURE WORK}

\section{Conclusions}

In Chapter II, the validity of the assumptions in Newman's equation used to interpret the results from the RDA were examined in this work. In particular, the assumption that the reactor dimensions do not impact the mass transfer of $\mathrm{H}^{+}$. Asymmetric flow under the disk and its impact on the reaction were investigated. This work led to the following conclusions:

1. A transition from an axisymmetric velocity profile occurs when $R_{\text {ehr }}$ is larger than 8,000 for Newtonian and non-Newtonian fluids.

2. Different flow regimes were found no to impact the slope of the rate of disk dissolution when plotted against rotational speeds.

3. Mass transfer of $\mathrm{H}^{+}$depends on the dimensions of the reactor and the location of the disk in the reactor.

4. The determination that, in Non-Newtonian fluids, the shear rate on the disk is not constant. This contradicts the assumption in the RDA equations currently used with non-Newtonian fluids.

5. The revelation that some RDA reactor dimensions are not large enough to prevent the impact of the boundaries on the mass transfer of $\mathrm{H}^{+}$to the disk. The larger the rate of disk dissolution, the larger the impact of the boundaries on the mass transfer is. 
6. The relative difference in the rock dissolved between the current reactor (A) and the larger reactor $(\mathrm{E})$ reached $13 \%$ in the simulations performed at $150^{\circ} \mathrm{F}$, which translated to an increase of $28 \%$ in the rate of mass transfer of $\mathrm{H}^{+}$to the disk. 
In Chapter III, the assumptions of laminar flow and uniform accessibility of the surface of the disk at commonly used RDA experimental conditions. Disks of calcite marble of three diameters- $0.72,1.11$, and 1.46 in.-were dissolved in $1 \mathrm{~N}$ and $15 \mathrm{wt} \% \mathrm{HCl}$ at temperatures of 73.4 and $100^{\circ} \mathrm{F}$, respectively. Experiments were performed at the disk rotational speeds of $207-1,555 \mathrm{rpm}$, maintaining the nitrogen pressure of $1,100-1,150 \mathrm{psig}$ in the reaction vessel. A computational fluid dynamics model was used to visualize the velocity under the disk. The following conclusions were reached:

1. With calcite disks dissolving in $15 \mathrm{wt} \% \mathrm{HCl}$ at $100^{\circ} \mathrm{F}$, transition to turbulent flow was observed at Reynolds numbers one order of magnitude lower than the universally accepted critical value of $3 \times 10^{5}$.

2. Laminar flow was lost at $587 \mathrm{rpm}$ for a $1.46 \mathrm{in}$. disk. The acid-diffusion coefficient is significantly overestimated if the experimental data are obtained in transition or turbulent flow regimes.

3. Dissolution in low-reactivity systems- $1 \mathrm{~N} \mathrm{HCl}$ at $73.4^{\circ} \mathrm{F}$ - reveals that in spite of the loss of the uniform accessibility of the disk surface, Levich's (1962) theory applies without noticeable error.

4. The critical Reynolds number for the flow at the surface of the disk is in the range $1-2 \times 10^{4}$

5. After dissolution tests, the appearance of each disk can indicate the flow regime (laminar or transition to turbulent). 
In Chapter IV, a computational fluid dynamics (CFD) model was developed to predict the diffusion coeffeceint and reaction rate coefficient from the RDA. More specifically, the current work proposes the first model to predict the reaction rate coefficient in the $\mathrm{HCl}$ calcite reaction from RDA experiments for concentrations up to $15 \mathrm{wt} \% \mathrm{HCl}$ and temperatures up to $275^{\circ} \mathrm{F}$. The following conclusions were reached:

1. A computationally efficient Gaussian process-based surrogate model was constructed for the original CFD model to accurately represent the reaction in the RDA with four orders of magnitude saving in computational time.

2. The developed calibrated surrogate model is able to predict the diffusion coefficient with an improvement in prediction accuracy obtained through experimental validation of $63 \%$.

\section{Recommendations}

Based on the results from the current work, the following recommendations are suggested for RDA operations:

1. The rate of $\mathrm{Ca}^{+2}$ dissolution needs to be corrected based on the reactor dimensions and acid strength to mitigate boundary effects. For reactor A dimensions and disk dissolution ranging between $3.0 \mathrm{E}-6$ and $3.9 \mathrm{E}-6 \mathrm{gmol} / \mathrm{cm}^{2} \bullet$, a correction factor ranging between 1.1 and 1.28 should be applied.

2. In order to reduce the impact of the boundaries of the reactor on the mass transfer of $\mathrm{H}^{+}$to the disk, a reactor with $13.4 \mathrm{~cm}$ height and $11.4 \mathrm{~cm}$ diameter should be used for disk diameter $3.81 \mathrm{~cm}$ and acid viscosity higher than $0.5 \mathrm{cp}$. 
3. This work was performed on marble, and as a result, an improved model to calculate the rate of mass transfer of $\mathrm{H}^{+}$to different disk types with fewer assumptions needs to be developed.

4. To obtain more experimental data points by extending the range of the disk rotational speeds in the laminar regime, disks of smaller diameters should be used, i.e. $0.75 \mathrm{in}$.

Based on the results from the current work, the added impacts of this work to acidizing are:

1. Calculating the diffusion coefficient will only require one experiment in contrast to the classical method that requires a minimum of three experiments, which saves six hours of labor.

2. Being the first model to predict reaction kinetics using the amount of rock dissolved instead of the concentration of $\mathrm{Ca}^{+}$ion in the effluent of the RDA experiments. The conventional method requires inductively coupled plasma, which is not readily available, while the proposed model only requires a scale to measure the amount of rock dissolved.

\section{Future Work}

Based on this work, several avenues can be expanded upon in the following ways: This work is the first step in building a multi-scale system that uses the results from RDA, coreflood, and well acidizing experiments and simulations to accurately predict reaction kinetics and the amount of rock dissolved at different scales. Both experimental and 
simulation works are needed at different scales to optimize the amount and rate of acid used in the field. Specifically:

1. Simulation advances are needed to properly simulate the transition between laminar and turbulent flow and its impact on rock dissolution.

2. Besides the Gaussian process, other machine learning algorithms should be tested and compared to provide the optimum model for dissolution simulation. 


\section{REFERENCES}

Abdelgawad, Z., Mahmoud. M., and Hussein, I. 2018. Stimulation of High Temperature Carbonate Gas Reservoirs Using Seawater and Chelating Agents: Reaction Kinetics. Journal of Natural Gas Science and Engineering 55: 595-605. https://doi.org/10.1016/j.jngse.2017.06.020.

Albery, W. J. and Bruckenstein, S. 1983. Uniformly Accessible Electrodes. Journal of Electroanalytical Chemistry 144 (1-2): 105-112. https://doi.org/10.1016/S0022$\underline{0728(83) 80148-X .}$.

Aldakkan, B., Gomaa, A. M., Cairns, A. J. et al. 2018. Low Viscosity Retarded Acid System: A Novel Alternative to Emulsified Acids. Paper presented at the SPE Kingdom of Saudi Arabia Annual Technical Symposium and Exhibition, Dammam, Saudi Arabia, 23-26 April. SPE-192175-MS. https://doi.org/10.2118/192175-MS.

Ali, M. T., Ezzat, A. A., and Nasr-El-Din, H. A. 2019. A Model to Simulate Matrix-Acid Stimulation for Wells in Dolomite Reservoirs with Vugs and Natural Fractures. SPE J. SPE-199341-PA https://doi.org/10.2118/199341-pa.

Ali, M. T. and Nasr-El-Din, H. A. 2019. A Robust Model to Simulate Dolomite-Matrix Acidizing. SPE Prod \& Oper 34 (01): 109-129. SPE-191136-PA. https://doi.org/10.2118/191136-pa.

Alkattan, M., Oelkers, E., Dandurand, J. et al. 1998. An Experimental Study of Calcite and Limestone Dissolution Rates as a Function of $\mathrm{pH}$ from -1 to 3 and Temperature from 25 to 80 C. Chemical Geology 151 (1-4): 199-214. https://doi.org/10.1016/s0009-2541(98)00080-1.

Alkhaldi, M. H., Sarma, H. K., and Nasr-El-Din, H. A. 2010. Diffusivity of Citric Acid During its Reaction with Calcite. J Can Pet Technol 49 (8): 43-52. https://doi.org/10.2118/139570-PA.

Anderson, M.S. 1991. Reactivity of San Andres Dolomite. SPE Prod Eng 6 (02): 227232. SPE-20115-PA. https://doi.org/10.2118/20115-pa.

Arakaki, T. and Mucci, A. 1995. A continuous and mechanistic representation of calcite reaction-controlled kinetics in dilute solutions at $25^{\circ} \mathrm{C}$ and $1 \mathrm{~atm}$ total pressure. Aquatic Geochemistry 1 (1): 105-130. https://doi.org/10.1007/bf01025233.

Arslan, E., Sokhanvarian. K, Nasr-El-Din, H. A. et al 2017. Reaction Rate of a Novel InSitu Generated $\mathrm{HCl}$ Acid and Calcite. Presented at the SPE Annual Technical 
Conference and Exhibition, San Antonio, Texas, 9-11 October. https://doi.org/10.2118/187059-ms.

Aubry, N. 1998. Transition to Turbulence on a Rotating Flat Disk. Physics of Fluids 6: 2800-2814. https://doi.org/10.1063/1.868168.

Bayarri, M. J., Berger, J. O., Paulo, R. et al. 2007. A Framework for Validation of $\begin{array}{lllll}\text { Computer } & \text { Models. } & \text { Technometrics } & 49 & \text { (2): 138-154. }\end{array}$ https://doi.org/10.1198/004017007000000092.

Balachandar, S., Streett, C., and Malik, M. 1992. Secondary Instability in Rotating-Disk Flow. Journal of Fluid Mechanics 242: 323-347. https://doi.org/10.1017/S0022112092002398.

Baune, M. 2002. Coupling of Chemical and Hydrodynamic Instabilities at the Electrochemical Dissolution of Metals. PhD dissertation, University of Bremen, Bremen, Germany (September 2002).

Bird, R. B. and Hassager, O. 1987. Dynamics of Polymeric Liquids, second edition. New Jersey: Wiley.

Boomer, D. R., McCune, C. C., and Fogler, H. S. 1972. Rotating Disk Apparatus for Reaction Rate Studies in Corrosive Liquid Environments. Review of Scientific Instruments $\mathbf{4 3}$ (2): 225-229. https://doi.org/10.1063/1.1685599.

Brown, D. A. and Atamturktur, S. 2018. Nonparametric Functional Calibration of Computer Models. Statistica Sinica 28 (2): 721742. https://doi.org/10.5705/ss.202015.0344.

Bruckenstein, S. and Miller, B. 1977. Unraveling Reactions with Rotating Electrodes. Accounts of Chemical Research 84 (2): 54-61. https://doi.org/10.1021/ar50110a004.

Chang, F. and Abbad, M. 2011. Modelling Mass Transfer in a Rotating Disk Reaction Vessel. KSG: 1-17.

Chin, D. and Litt, M. 1972. An Electrochemical Study of Flow Instability on a Rotating Disk. Journal of Fluid Mechanics 54 (4): 613-625. https://doi.org/10.1017/S0022112072000904.

Cifuentes, A. O. and Kalbag, A. 1992. A Performance Study of Tetrahedral and Hexahedral Elements in 3-D Finite Element Structural Analysis. Finite Elements in Analysis and Design 12 (3-4): 313-318. https://doi.org/10.1016/0168-874x(92)90040$\mathrm{i}$. 
Conway, M. W., Asadi, M., Penny, G. S. et al. 1999. A Comparative Study of Straight/Gelled/Emulsified Hydrochloric Acid Diffusivity Coefficient Using Diaphragm Cell and Rotating Disk. Presented at the SPE Annual Technical Conference and Exhibition, Houston, Texas, 3-6 October. https://doi.org/10.2118/56532-ms.

Corke, T. C., Matlis, E. H., and Othman, H. 2007. Transition to Turbulence in RotatingDisk Boundary Layers-Convective and Absolute Instabilities. Journal of Engineering Mathematics 57: 253-272. https://doi.org/10.1007/s10665-006-9099-1.

Clarkson, M. H., Chin, S. C., and Shacter, P. 1980. Flow Visualization of Inflexional Instabilities on a Rotating Disk. 18th Aerospace Sciences Meeting. https://doi.org/10.2514/6.1980-279.

Daguenet, M. 1968. Etude du Transport de Matiere en Solution, a L'aide des Electrodes a Disque et a Anneau Tournants. International Journal of Heat and Mass Transfer 11 (11): 1581-1596. https://doi.org/10.1016/0017-9310(68)90040-9.

Dong, Q., Santhanagopalan, S., and White, R. E. 2008. A Comparison of Numerical Solutions for the Fluid Motion Generated by a Rotating Disk Electrode. Journal of The Electrochemical Society 155 (9): B963-B968. https://doi.org/10.1149/1.2953519.

Duck, P. 1986. On the Flow between Two Rotating Shrouded Discs. Computers and Fluids 14 (3): 183-196. https://doi.org/10.1016/0045-7930(86)90020-4.

Economides, M. J. and Nolte, K. G. 1989. Acidizing Physics. Reservoir Stimulation, second edition, Chap 13. New Jersey: Prentice Hall, Englewood Cliffs.

Ellison, B. T. and Cornet, I. 1971. Mass Transfer to a Rotating Disk. Journal of the Electrochemical Society 118 (1): 68-72. https://doi.org/10.1149/1.2407954.

Ezzat, A. A., Pourhabib, A., and Ding, Y. 2018. Sequential Design for Functional Calibration of Computer Models. Technometrics 60 (3): 286-296. https://doi.org/10.1080/00401706.2017.1377638.

Ezzat, A. A., Tang, J. and Ding, Y. 2020. A Model-Based Calibration Approach for Structural Fault Diagnosis Using Piezoelectric Impedance Measurements and a Finite Element Model. Structural Health Monitoring 19 (6): 1839-1855. https://doi.org/10.1177/1475921719901168.

Finneran, D. W. and Morse, J. W. 2009. Calcite Dissolution Kinetics in Saline Waters.

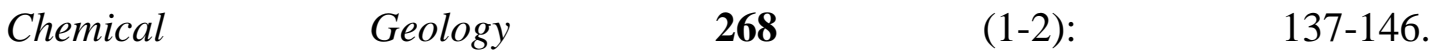
https://doi.org/10.1016/j.chemgeo.2009.08.006. 
Fredd, C. N. 1997. The Influence of Transport and Reaction of Wormhole Formation in Carbonate Porous Media: A Study of Alternative Stimulation Fluids. PhD dissertation, University of Michigan, Michigan.

Fredd, N. and Fogler, H. 1998. The Kinetics of Calcite Dissolution in Acetic Acid Solutions. Chemical Engineering Science $\mathbf{5 3}$ (22): 3863-3874. https://doi.org/10.1016/s0009-2509(98)00192-4.

Frueh, W. and Read, P. 1999. Experiments on a Barotropic Rotating Shear Layer. Part 1. Instability and Steady Vortices. J. Fluid Mechanics 383: 143-173. https://doi.org/10.1017/s0022112098003930.

Gdanski, R.D. and Norman, L.R. 1986. Using the Hollow-Core Test to Determine Acid Reaction Rates. SPE Prod Eng 1 (02): 111-116. SPE-12151-PA. https://doi.org/10.2118/12151-pa.

Gledhill, D. K. and Morse, J. W. 2006. Calcite Dissolution Kinetics in Na-Ca-Mg-Cl Brines. Geochimica et Cosmochimica Acta 70 (23): 5802-5813. https://doi.org/10.1016/j.gca.2006.03.024.

Gregory, D. P. and Riddiford, A. C. 1956. Transport to a Surface of a Rotating Disk. J. Chem. Soc. 6:3756-3764. https://doi.org/10.1039/jr9560003756.

Gregory, N., Stuart, J. T., and Walker, W. S. 1955. On the Stability of Three-Dimensional Boundary Layers with Application to the Flow Due to a Rotating Disk. Philosophical Transactions of the Royal Society of London. Series A, Mathematical and Physical Sciences 248 (943): 155-199. https://doi.org/10.1098/rsta.1955.0013.

Gutjahr, A., Dabringhaus, H., and Lacmann, R. 1996. Studies of the growth and dissolution kinetics of the $\mathrm{CaCO}_{3}$ polymorphs calcite and aragonite I. Growth and dissolution rates in water. J. Crystal Growth 158 (3): 296-309. https://doi.org/10.1016/0022-0248(95)00446-7.

Hall-Thompson, B., Ernesto, A. R., Abdulrahman, N. et al. 2020. Acid Stimulation-Best Practices for Design, Selection and Testing of Acid Recipes in Low Permeability Carbonate Reservoirs. Paper presented at the International Petroleum Technology Conference, Dhahran, Kingdom of Saudi Arabia, 13-15 January. https://doi.org/10.2523/IPTC-19690-MS.

Hansford, G. S. and Litt, M. 1968. Mass Transport from a Rotating Disk into Power-Law Liquids. Chem. Eng. Sci. 23 (8): 849-864. https://doi.org/10.1016/00092509(68)80020-x. 
Harris, J. H., Thomas, P. J., and Garrett, S. J. 2012. On the Stability of Flows over Rough Rotating Disks. 42nd AIAA Fluid Dynamics Conference and Exhibition. https://doi.org/10.2514/6.2012-3075.

Heidaryan, E. 2019. A Note on Model Selection Based on the Percentage of AccuracyPrecision. Journal of Energy Resources Technology 141 (4). https://doi.org/10.1115/1.4041844.

Ivanishin, I., Kotb, A., and Nasr-El-Din, H.A., 2021. Turbulence Leads to Overestimation of the Acid-Diffusion Coefficient at Typical Experimental Conditions Using the Rotating Disk Apparatus. Journal of Petroleum Science and Engineering. 205. https://doi.org/10.1016/j.petrol.2021.108805.

Jang, D. S., Jetli, R., and Acharya, S. 1986. Comparison of the PISO, SIMPLER, and SIMPLEC Algorithms for the Treatment of the Pressure-Velocity Coupling in Steady Flow Problems. Numerical Heat Transfer 10 (3): 209-228. https://doi.org/10.1080/10407788608913517.

Jarre, S., Le Gal, P., and Chauve, M. P. 1996. Experimental Study of Rotating Disk Instability. I. Natural Flow. Physics of Fluids $\mathbf{8}$ (2): 496-508. https://doi.org/10.1063/1.868803.

Kennedy, M. C. and O'Hagan, A. 2001. Bayesian calibration of computer models. Royal Statistical Society, Series B 63 (3):425-464. https://doi.org/10.1111/1467-9868.00294.

Khalid, M. A., Sultan, A., and Qiu, X. 2015. Revisiting Reaction Kinetics and Diffusion Rate of Dolomitic Rock with HCl. Paper presented at the SPE North Africa Technical Conference and Exhibition, Cairo, Egypt, 14-16 September. SPE-175832-MS. https://doi.org/10.2118/175832-MS.

Kobayashi, R., Kohama, Y., and Takamadate, C. 1980. Spiral Vortices in Boundary Layer Transition Regime on a Rotating Disk. Acta Mechanica 35: 71-82. https://doi.org/10.1007/BF01190058.

Kohama, Y. 1984. Study on Boundary Layer Transition of a Rotating Disk. Acta Mechanica 50: 193-199. https://doi.org/10.1007/BF01170959.

Kohama, Y. and Sudaf, K. 1992. Crossflow Instability in a Spinning Disk Boundary Layer. AIAA Journal 31 (1): 212-14. https://doi.org/10.2514/3.11344.

Kotb, A., Ali, M., Ezzat, A. et al. 2018. A Computational Fluid Dynamics Model for Simulating the Rotating Disk Apparatus. Presented at the SPE International Heavy Oil 
Conference and Exhibition, Kuwait City, Kuwait, 10-12 December. https://doi.org/10.2118/193739-ms.

Kotb, A., Ezzat, A., Ali, M., et al. 2021. A Calibrated Computational Fluid Dynamics Model for Simulating the Rotating Disk Apparatus. SPE J. SPE-193739-PA https://doi.org/10.2118/193739-PA.

Kotb, A. and Nasr-El-Din, H. 2020. New Insights into Mass Transfer When Using the Rotating Disk Apparatus for Newtonian and Non-Newtonian Fluids. SPE J. SPE201246-PA https://doi.org/10.2118/201246-PA.

Kreith, F., Taylor, J. H., and Chong, J. P. 1959. Heat and Mass Transfer from a Rotating Disk. Journal of Heat Transfer 81 (2): 95-103. https://doi.org/10.1115/1.4008145.

Kung, M. 1998. Flow and Reaction of Weak Acids in Carbonate Porous Media. MS Thesis, University of Michigan, Ann Arbro, Michigan.

Lehmkuhl, G. and Hudson, J. 1971. Flow and Mass Transfer near an Enclosed Rotating Disk: Experiment. Chem Eng Sci 26 (10): 1601-1613. https://doi.org/10.1016/00092509(71)86050-5.

Levich, V. G. 1942. The Theory of Concentration Overpotential. Acta Physicochimica URSS 17: 257-307.

Levich, V. G. 1962. Physicochemical Hydrodynamics, first edition. New Jersey: PrenticeHall Inc., Englewood Cliffs.

Li, N., Zeng, F., Li, J. et al. 2016. Kinetic Mechanics Of The Reactions between HCl/HF Acid Mixtures and Sandstone Minerals. J Natural Gas Sci. \& Eng. 34: 792-802. https://doi.org/10.1016/j.jngse.2016.07.044.

Liao, Y., Zhang, D., Peng, J. et al. 2017. Measurement of Reaction Rate of Gelled Acids and Calcite with the Rotating Disk Apparatus. Natural Resources 8 (08): 559-568. https://doi.org/10.4236/nr.2017.88035.

Lingwood, R. J. 1996. An Experimental Study of Absolute Instability of the RotatingDisk Boundary-Layer Flow. Journal of Fluid Mechanics 314: 373-405. https://doi.org/10.1017/S0022112096000365.

Litt, M. and Serad, G. 1964. Chemical Reactions on a Rotating Disk. Chem. Eng. Sci. 19 (11): 867-884. https://doi.org/10.1016/0009-2509(64)85065-x. 
Lobo, V. M. M., Helena, M., and Teixeira, S. F. 1979. Diffusion Coefficients in Aqueous Solutions of Hydrochloric Acid at 298 K. Electrochimica Acta 24 (5): 565-567. https://doi.org/10.1016/0013-4686(79)85033-1.

Lund, K., Fogler, S. H., McCune, C. C. et al. 1973a. Kinetic Rate Expressions for Reactions of Selected Minerals with $\mathrm{HCl}$ and HF Mixtures. Presented at the SPE Oilfield Chemistry Symposium, Denver, 24-25 May. https://doi.org/10.2523/4348$\underline{\mathrm{ms}}$.

Lund, K., Fogler, S. H., and McCune, C. C. 1973b. Acidization-I. The dissolution of dolomite in hydrochloric acid. Chem Eng Sci 28 (3): 691-700. https://doi.org/10.1016/0009-2509(77)80003-1.

Lund, K., Fogler, S. H., McCune, C. C. et al. 1975. Acidization-II. The dissolution of calcite in hydrochloric acid. Chem Eng Sci 30 (8): 825-835. https://doi.org/10.1016/0009-2509(75)80047-9.

Malik, M. R., Wilkinson, S. P., and Orszag, S. A. 1981. Instability and Transition in Rotating Disk Flow. AIAA Journal 19 (9): 1131-1138. https://doi.org/10.2514/3.7849.

Malik, M. R. 1986. The Neutral Curve for Stationary Disturbances in Rotating-disk Flow. Journal of Fluid Mechanics 164: 275287. https://doi.org/10.1017/S0022112086002550.

McNaught, A. D. and Alan, D. M. 1997. Compendium of Chemical Terminology. Oxford: Blackwell Science.

Mishra, P. and Singh, P. C. 1978. Mass Transfer from Rotating Disk to Non-Newtonian Fluids. Chem. Eng. Sci. 33 (11): 1463-1470. https://doi.org/10.1016/00092509(78)85195-1.

Mohr, C. M. and Newman, J. 1976. Mass Transfer to a Rotating Disk in Transition Flow. Journal of the Electrochemical Society 123 (11): 1687-1691. https://doi.org/10.1149/1.2132668.

Nanis, L. and Klein, I. 1972. Transient Mass Transfer at the Rotating Disk Electrode. Journal of The Electrochemical Society 119 (12): 1683-1687. https://doi.org/10.1149/1.2404070.

Nasr-El-Din, H. A., Al-Mohammed, A. M., and Al-Aamri, A. 2008. Reaction of Gelled Acids with Calcite. SPE Prod \& Oper 23 (03): 353-361. https://doi.org/10.2118/103979-pa. 
Newman, J. 1966. Schmidt Number Correction for the Rotating Disk. J. Phys Chem 70 (4): 1327-1328. https://doi.org/10.1021/j100876a509.

Nishikata, E., Ishii, T., and Ohta, T. 1981. Viscosities of Aqueous Hydrochloric Acid Solutions, and Densities and Viscosities of Aqueous Hydroiodic Acid Solutions. $J$. Chem. Eng. Data 26 (3): 254-256. https://doi.org/10.1021/je00025a008.

Panga, M. K. R., Ziauddin M., and Balakotaiah V, 2005. Two-Scale Continuum Model for Simulation of Wormholes in Carbonate Acidization. AIChE journal 51(12): 32313248. https://doi.org/10.1002/aic.10574.

Peng, C., Crawshaw, J. P., Maitland, G. C. et al. 2015. Kinetics of Calcite Dissolution in $\mathrm{CO}_{2}$-Saturated Water at Temperatures between (323 and 373) $\mathrm{K}$ and Pressures Up to $13.8 \quad \mathrm{MPa} \quad$ Chemical Geology 403: https://doi.org/10.1016/j.chemgeo.2015.03.012.

Plumlee, M., Joseph, V. R., and Yang, H. 2016. Calibrating Functional Parameters in the Ion Channel Models of Cardiac Cells. J American Statistical Association 111 (514): 500-509. https://doi.org/10.1080/01621459.2015.1119695.

Pourhabib, A., Huang, J. Z., Wang, K. et al. 2015. Modulus Prediction of Buckypaper Based on Multi-Fidelity Analysis Involving Latent Variables. IIE Transactions 47 (2): 141-152. https://doi.org/10.1080/0740817x.2014.917777.

Prakongpan, S., Higuchi, W. I., Kwan, K. H. et al. 1976. Dissolution Rate Studies of Cholesterol Monohydrate in Bile Acid-Lecithin Solutions Using the Rotating-Disk Method. J. Pharmaceutical Sciences $65 \quad$ (5): 685-689. https://doi.org/10.1002/jps.2600650514.

Qiu, X., Khalid, M. A., and Sultan, A. 2015. How to Determine True Acid Diffusion Coefficient to Optimize Formation Damage Treatment. Presented at the SPE European Formation Damage Conference and Exhibition, Budapest, Hungary, 3-5 June. https://doi.org/10.2118/174241-ms.

Rabie, A. I., Shedd, D. C., and Nasr-El-Din, H. A. 2014. Measuring the Reaction Rate of Lactic Acid with Calcite and Dolomite by Use of the Rotating-Disk Apparatus. SPE J. 19 (6): 1192-1202. https://doi.org/10.2118/140167-pa.

Ramos, A. and Simoes, J. A. 2006. Tetrahedral Versus Hexahedral Finite Elements in Numerical Modelling of the Proximal Femur. Medical Engineering \& Physics 28 (9): 916-924. https://doi.org/10.1016/j.medengphy.2005.12.006. 
Rashed, M. K., Abdulbari, H. A., Salled, M. A. et al. 2016. Rotating Disc Apparatus: Types, Developments and Future Applications. Modern Applied Science 10 (8): 198229. https://doi.org/10.5539/mas.v10n8p198.

Rasmussen, C. and Williams, K. 2006. Gaussian Processes for Machine Learning. The MIT Press.

Roberts, L. D. and Guin, J. A. 1974. The Effect of Surface Kinetics in Fracture Acidizing. SPE J. 14 (4): 385-395. https://doi.org/10.2118/4349-pa.

Roberts, L. D. and Guin, J. A. 1975. A New Method for Predicting Acid Penetration Distance. SPE J. 15 (4): 277-286. https://doi.org/10.2118/5155-pa.

Rogers, G. T. and Taylor K. J. 1963. Effect of Small Protrusions on Mass Transport to a Rotating-Disk Electrode. Nature 200: 1062-1064. https://doi.org/10.1038/2001062a0.

Rozieres, J. D., Chang, F. F., and Sullivan, R. B. 1994. Measuring Diffusion Coefficients in Acid Fracturing Fluids and Their Application to Gelled and Emulsified Acids. Presented at the SPE Annual Technical Conference and Exhibition, New Orleans, 2528 September. https://doi.org/10.2118/28552-ms.

Santner, T. J., Williams, B. J., and Notz, W. I. 2003. The Design and Analysis of Computer Experiments, first edition. New Yotk: Springer Verlag. https://doi.org/10.1007/978-14757-3799-8.

Sayed, M., Cairns, A. J., and Sahu, Q. 2020. Low-Viscosity Acid Platform: Benchmark Study Reveals Superior Reaction Kinetics at Reservoir Conditions. Presented at the International Petroleum Technology Conference, Dhahran, KSA, 13-15 January. https://doi.org/10.2523/iptc-20282-ms.

Sjöberg, E. L. 1976. A fundamental equation for calcite dissolution kinetics. Geochimica et Cosmochimica Acta 40 (4): 441-447. https://doi.org/10.1016/0016-7037(76)90009$\underline{0}$.

Sjöberg, E. L. and Rickard, D. T. 1984. Temperature dependence of calcite dissolution kinetics between 1 and $62^{\circ} \mathrm{C}$ at $\mathrm{pH} 2.7$ to 8.4 in aqueous solutions. Geochimica et Cosmochimica Acta 48 (3): 485-493. https://doi.org/10.1016/0016-7037(84)90276-x.

Taylor, K. C., Al-Ghamdi, A. W. H., and Nasr-El-Din, H. A. 2004. Effect of Additives on the Acid Dissolution Rates of Calcium and Magnesium Carbonates. SPE Prod and Fac 19 (3): 122-127. SPE-80256-PA. https://doi.org/10.2118/80256-PA. 
Taylor, K. C. and Nasr-El-Din, H. A. 2009. Measurement of Acid Reaction Rates with the Rotating Disk Apparatus. J Can Pet Technol 48 (6): 66-70. https://doi.org/10.2118/09$\underline{06-66 .}$

Vahdat, N. and Newman, J. 1973. Corrosion of an Iron Rotating Disk. Journal of The Electrochemical Society 120 (12): 1682-1686. https://doi.org/10.1149/1.2403329.

Vo, T., Montabone, L., and Sheard, G. J. 2014. Linear Stability Analysis of a Shear Layer Induced by Differential Coaxial Rotation within a Cylindrical Enclosure. $J$. Fluid Mechanics 738: 299-334. https://doi.org/10.1017/jfm.2013.594.

Vo, T., Montabone, L., and Sheard, G. J. 2015. Effect of Enclosure Height on the Structure and Stability of Shear Layers Induced by Differential Rotation. J. Fluid Mechanics 765: 45-81. https://doi.org/10.1017/jfm.2014.727.

Welton, T. D. and Domelen, M. S. 2008. High-Viscosity-Yield Acid Systems for HighTemperature Stimulation. SPE Prod \& Oper 23 (03): 177-183. https://doi.org/10.2118/98237-pa.

Wilkinson, S. P. and Malik, M. R. 1985. Stability Experiments in the Flow over a Rotating Disk. AIAA Journal 23 (4): 588-595. https://doi.org/10.2514/3.8955.

Zhang, Y., Yang, S., Zhang, S. et al. 2014. Wormhole Propagation Behavior and its Effect on Acid Leakoff Under in Situ Conditions in Acid Fracturing. Transport in porous media 101 (1): 99-114. https://doi.org/10.1007/s11242-013-0233-z. 